\title{
A noncooperative approach to bankruptcy problems with an endogenous estate
}

Citation for published version (APA):

Karagozoglu, E. (2010). A noncooperative approach to bankruptcy problems with an endogenous estate. METEOR, Maastricht University School of Business and Economics. METEOR Research Memorandum No. 027 https://doi.org/10.26481/umamet.2010027

Document status and date:

Published: 01/01/2010

DOI:

10.26481/umamet.2010027

Document Version:

Publisher's PDF, also known as Version of record

\section{Please check the document version of this publication:}

- A submitted manuscript is the version of the article upon submission and before peer-review. There can be important differences between the submitted version and the official published version of record.

People interested in the research are advised to contact the author for the final version of the publication, or visit the DOI to the publisher's website.

- The final author version and the galley proof are versions of the publication after peer review.

- The final published version features the final layout of the paper including the volume, issue and page numbers.

Link to publication

\footnotetext{
General rights rights.

- You may freely distribute the URL identifying the publication in the public portal. please follow below link for the End User Agreement:

www.umlib.nl/taverne-license

Take down policy

If you believe that this document breaches copyright please contact us at:

repository@maastrichtuniversity.nl

providing details and we will investigate your claim.
}

Copyright and moral rights for the publications made accessible in the public portal are retained by the authors and/or other copyright owners and it is a condition of accessing publications that users recognise and abide by the legal requirements associated with these

- Users may download and print one copy of any publication from the public portal for the purpose of private study or research.

- You may not further distribute the material or use it for any profit-making activity or commercial gain

If the publication is distributed under the terms of Article $25 \mathrm{fa}$ of the Dutch Copyright Act, indicated by the "Taverne" license above, 


\section{Maastricht University}

Emin Karagözoğlu

A Noncooperative Approach to Bankruptcy Problems with an Endogenous Estate

$\mathrm{RM} / 10 / 027$

\section{METEOR}

Maastricht University School of Business and Economics

Maastricht Research School of Economics

of Technology and Organization

P.O. Box 616

NL - 6200 MD Maastricht

The Netherlands 


\title{
A Noncooperative Approach to Bankruptcy Problems with an Endogenous Estate*
}

\author{
Emin Karagözoğlu ${ }^{\dagger}$ \\ Maastricht University, Department of Economics
}

May, 2010

\begin{abstract}
We introduce a new class of bankruptcy problems in which the value of the estate is endogenous and depends on agents' investment decisions. There are two investment alternatives: investing in a company and becoming a shareholder (risky asset) and depositing money into a savings account (risk-free asset). Bankruptcy is a possible event only for the risky asset. We define a game between agents each of which aims to maximize his expected payoff by choosing an investment alternative and a company management which aims to maximize profits by choosing a bankruptcy rule. There are two types of agents in our basic model, who are differentiated by their incomes. We, first, consider three well-known bankruptcy rules: the proportional rule, the constrained equal awards rule and the constrained equal losses rule. We show that there always exists a pure strategy subgame perfect Nash equilibrium, which involves the proportional rule. This result is independent of the income distribution in the economy and holds even under one-sided uncertainty on the income distribution. Moreover, if the company optimally chooses the return rate to be paid to investors, the unique subgame perfect Nash equilibrium involves the proportional rule. We also extend our model in two dimensions: (i) to a larger set of rules containing the Talmud rule and (ii) to two companies competing over potential investors.
\end{abstract}

JEL Codes: C720, D710.

Keywords: Bankruptcy Problems, Subgame Perfect Equilibrium, Proportional Rule, Constrained Equal Awards Rule, Constrained Equal Losses Rule.

\footnotetext{
${ }^{*}$ I would like to thank my advisor, Bettina-E. Klaus, for her constant support, encouragement and many helpful comments. I would also like to thank Carlos Alós-Ferrer, Salvador Barberá, Kristof Bosmans, Refet Gürkaynak, Kevin Hasker, Jean-Jacques Herings, Biung-Ghi Ju, Çağatay Kayı, Özgür Kıbrıs, Hülya Kuzucu Eraslan, Maria Montero, Juan D. Moreno-Ternero, Hervé Moulin, Antonio Nicoló, Hans Peters, Alex Possajennikov, Arno Riedl, Rene Saran, William Thomson, Stef Tijs, Gisèle Umbhauer, Peyton Young and conference, workshop and seminar participants at $5^{\text {th }}$ Murat Sertel Student Workshop, $28^{\text {th }}$ Annual Meeting of the European Public Choice Society, $3^{r d}$ Economic Design \& Collective Choice Workshop, $9^{\text {th }}$ International Meeting of Society for Social Choice and Welfare, $1^{\text {st }}$ Doctoral Workshop in Game Theory, $6^{\text {th }}$ Conference on Economic Design, $2^{\text {nd }}$ METEOR Ph.D. Colloquium, Nottingham School of Economics, Bilkent University, Sabancı University and Boğaziçi University for their comments. All remaining errors are mine.

${ }^{\dagger}$ Contact Info: Maastricht University, Department of Economics, P.O. Box 616, 6200 MD, Maastricht, The Netherlands. Phone : +31 (0) 433883925 Fax: +31 (0) 4338848 78. Email: E.Karagozoglu@maastrichtuniversity.nl.
} 


\section{Introduction}

\subsection{Motivation}

As early as 1985, Young argued that the incentives of agents should be incorporated into costsharing models. ${ }^{1}$ He summarizes the motivation for this argument in the following passage:

"The problem with these approaches (ad-hoc cost sharing mechanisms) is that they ignore the problem of motivation: why should a customer pay his allocated cost if it exceeds the benefits or the alternative cost of obtaining the service by some other means? This incentive argument is especially critical in the case of voluntary association such as a club, a public consortium, or a cartel, where the partners must first agree in the manner of splitting the costs and benefits before they can proceed with the enterprise."

Thomson (2003) also addressed the need to combine noncooperative and market-based approaches to analyze bankruptcy problems in the following paragraph:

"An important question that we will not address is the extent to which the choice of particular division rules affects agents' incentives to make commitments that one party may in the end be unable to honor. In the context of bankruptcy, these are the incentives to loan and to borrow. In many of the other applications, the parameters of the problems to be solved also result from decisions that agents have made, and whatever rule is used at the division stage will in general have had an effect on these earlier choices. In order to handle these kinds of issues, we would need to embed division rules in a more complete model in which risk-taking, effort, and other variables chosen by agents, such as lenders, borrowers, tax payers, government agencies and others, are explicitly described, stochastic returns to economic activities are factored in, and so on."

In this paper, we introduce a new class of bankruptcy problems in which the value of the estate is endogenous and depends on agents' investment decisions that also determine their claims. Our theoretical framework incorporates important economic factors such as the income distribution, stochastic returns of risky investment projects, and the return on a risk-free outside option. Our model is motivated by the following facts: (i) In bankruptcy situations, agents might act strategically and in line with their incentives, (ii) bankruptcy might occur following an investment decision with stochastic outcomes, (iii) the choice of the bankruptcy rule and investment decisions have impacts on each other through incentives, (iv) the claims distribution might have an impact on agents' decisions if there are peer effects, and (v) many real bankruptcy situations involve payments (to shareholders, lenders, partners etc.), which are not respected by the borrower.

In our base model, there are two investment alternatives: investing in a company and becoming shareholder (risky asset) or depositing money into a savings account (risk-free asset). Bankruptcy is a possible event only for the risky asset. We define a game between agents each of which aims to maximize his expected payoff by choosing an investment alternative and a company management which aims to maximize the investment in the company by choosing a bankruptcy rule. This setup is also in line with some recent suggestions in favor of a more liberal bankruptcy law, which would provide a menu of rules and allows companies to choose one among them (see Hart, 2000). There are two types of agents in the base model, who are differentiated by their incomes. We, first, consider three well-known bankruptcy rules: the proportional rule, the constrained equal awards rule and the constrained equal losses rule. In the game, the company chooses the bankruptcy rule and later

\footnotetext{
${ }^{1}$ I would like to thank Peyton Young for referring me to this paper.
} 
all agents simultaneously choose whether to invest in the risky asset (i.e., the project initiated by the company) or the risk-free asset (savings account in a bank). Results in our base model provide a noncooperative support for the proportional rule. ${ }^{2}$ In particular, we show that there always exists a pure strategy subgame perfect Nash equilibrium that involves the proportional rule. This statement is not valid for the constrained equal awards or the constrained equal losses rules. The direct implication of this result is that the proportional rule never leads to an investment in the company that is lower than the one under the constrained equal awards rule or the constrained equal losses rule; and in some cases leads to an investment in the company strictly higher than the one under the two rules. Moreover, the result supporting the proportional rule is independent of the income distribution and holds even under one-sided uncertainty on the income distribution. We obtain even a stronger result in favor of the proportional rule as we endogenize the return rate to be paid to investors. In particular, if the company optimally chooses the return rate, the unique subgame perfect Nash equilibrium involves the proportional rule. We also extend our base model in two dimensions: (i) to a larger set of rules containing the Talmud rule, (ii) to two companies competing over potential investors.

\subsection{Overview of the Literature}

The bankruptcy problem was first introduced formally by O'Neill (1982). It describes a situation in which there is a perfectly divisible estate to be allocated to a finite number of agents, whose claims add up to an amount larger than the estate. ${ }^{3}$ A bankruptcy problem can be represented by a claims vector and an estate. A bankruptcy rule is a function that associates a division of the estate with every bankruptcy problem. Many real life situations such as distributing a will to inheritants, liquidating the assets of a bankrupt company, rationing, taxation, and sharing the costs of a public facility can be described using parsimonious bankruptcy models.

Following the introduction of the bankruptcy problem, most research on bankruptcy approaches the problem from a normative (axiomatic) perspective and aims to compare different rules by their properties. As Thomson (2003) shows, this normative approach was successful in determining strong contenders, i.e., a small set of bankruptcy rules with particularly desirable properties. The most prominent rules are the proportional rule, the constrained equal awards rule, the constrained equal losses rule and the Talmud rule. The proportional rule allocates the estate proportionally with respect to claims. The constrained equal awards rule allocates the estate as equal as possible taking claims as upper bounds and similarly the constrained equal losses rule allocates the shortage of the estate in an equal way (shares bounded below by zero). The Talmud rule behaves like the constrained equal awards rule when the estate is less than half of the total claims and like the constrained equal losses rule when the estate is more than half of the total claims. These rules will also be used in our research. For an extensive survey of the axiomatic literature, the reader is referred to Moulin (2002) and Thomson (2003; 2006).

Some researchers (Yaari \& Bar-Hillel, 1984; Moulin, 2000; and Herrero, Moreno-Ternero \& Ponti, 2010) offer informal characterizations of different circumstances in which a particular bank-

\footnotetext{
${ }^{2}$ For additional support for the proportional rule, we refer the reader to Chun (1988), Bergantiños \& Sanchez (2002), Chambers \& Thomson (2002), Ching \& Kakkar (2001), Chun \& Lee (2007), De Frutos (1999), Gächter \& Riedl (2005; 2006), Hougaard \& Østerdal (2005), Ju (2003), Ju, Miyagawa \& Sakai (2007), and Moreno-Ternero (2002; 2006; 2009).

${ }^{3}$ This corresponds to Chapter 7 bankruptcy in the US bankruptcy law.
} 
ruptcy rule is the most sensible one. Some examples are the use of the proportional rule in income taxation and in the allocation of a bankrupt firm's assets to shareholders, the constrained equal losses rule in the provision of health services, and the constrained equal awards rule in the allocation of a bankrupt bank's assets to depositors.

Still another approach to bankruptcy problems is the game theoretical approach. There are different strands within the game theoretical approach to bankruptcy problems. Historically, the first one is the cooperative game theoretical approach. This approach transforms the bankruptcy problem to a transferable utility game or a coalitional bargaining game and studies cooperative solution concepts such as the core, the kernel etc.; Aumann \& Maschler (1985), Young (1985), Curiel, Maschler \& Tijs (1987) and Dagan \& Volij (1993) are some of the papers that apply the cooperative game theoretical approach to analyze bankruptcy problems.

We know that incentives and strategic behavior play a significant role in real-life bankruptcy problems. Hence, the noncooperative game theoretical approach is also a natural and fruitful one. The noncooperative game theoretical approach models the bankruptcy problem as a noncooperative game among the claimants and studies equilibria of the game. This approach aims to provide a noncooperative support for bankruptcy rules. There are, to the best of our knowledge, only a few papers using this approach. O'Neill (1982), Chun (1989), Dagan, Serrano \& Volij (1997), Moreno-Ternero (2002), Herrero (2003), García-Jurado, González-Díaz \& Villar (2006), Chang \& $\mathrm{Hu}$ (2008), Ashlagi, Karagözoğlu \& Klaus (2008), and Atlamaz, Berden, Peters \& Vermeulen (2008) apply the noncooperative game theoretical approach to analyze bankruptcy problems. Ching \& Kakkar (2001), Araujo \& Páscoa (2002), and Ju \& Karagözoğlu (2009) approach the problem from a slightly different perspective by offering market based formulations.

The major motivation of all these studies is that when the authority does not have a priori preferences concerning the rule that will be implemented, it might resort to implementing a noncooperative game form (a set of rules or procedures) in which the strategic interactions of claimants determine the rule to be used in equilibrium. Depending on the strategic game form the policymaker implements, the resulting equilibrium rules may differ as the studies mentioned above show.

The paper most closely related to ours is Kibris \& Kibris (2008). They also analyze the investment implications of prominent bankruptcy rules. The major differences between our models can be listed as: (i) in our model, the bankruptcy rule decision is embedded in a sequential game, whereas in their paper compare investment volumes under different bankruptcy rules are compared, (ii) in our paper, agents invest either nothing or everything in the risky asset, whereas in their paper agents solve an optimization problem to determine this investment amount, (iii) in our paper results are valid for two types of agents, whereas in their paper, there are exactly two agents, (iv) in our paper, agents are risk neutral and there is a risk-free outside option, whereas in their paper, they allow for risk aversion and there is no outside option, and (v) we extend our model to contain a larger set of rules and two competing companies. As a result of similarities between our base models, some of our results are identical. For instance, in both papers, the proportional rule leads to more investment than the constrained equal awards rule. One major difference between results is that in our paper, the proportional rule leads to more investment than the constrained equal losses rule, whereas in their paper, this is not always valid. 


\subsection{Our Contribution}

Contributions of our paper can be listed as: (a) endogenizing the determination of the bankruptcy rule with a noncooperative procedure, (b) endogenizing the value of claims and the estate, (c) incorporating the well-known bankruptcy model into a context that involves decision-making under uncertainty and mimics a market environment, (d) offering, at least, a partial explanation to a real-life phenomenon, which is the use of the proportional rule in allocating a bankrupt company's assets to shareholders, and (e) providing a noncooperative framework in which the bankruptcy rule decision depends on both borrowers' (companies') and lenders (agents') incentives. Firstly, endogenously determined bankruptcy rules, claims and estates are new in the literature. In most of the papers on bankruptcy, the analysis is based on exogenously fixed bankruptcy rules, claims and estates. In real life, obviously agents' decisions and hence the value of claims and the estate depend on the bankruptcy rule and the choice of the bankruptcy rule depends, in turn, on agents' actions. Thus, the bankruptcy rule, claims and estate are all endogenously determined. Secondly, many real life instances that involve a bankruptcy problem also involve an investment decision under uncertainty. In our paper, we model the whole investment process at an earlier stage, i.e. before bankruptcy is realized. ${ }^{4}$ With this approach, we also incorporate factors that play important roles in real-life bankruptcy problems such as stochastic returns, risk, attitudes towards risk and income distribution into the bankruptcy problem. Our model gives, at least, a partial explanation for the popular use of the proportional rule in the liquidation process of a bankrupt company (i.e., its use in each priority class in sequential priority rules employed by many bankruptcy laws such as Chapter 11 of the US bankruptcy law). Finally, in all bankruptcy papers with noncooperative approaches, the strategic interaction takes place among claimants, whereas the bankruptcy rule decision is influenced by both lenders' and borrower's interests in our paper. It is determined as a result of a sequential game played among the lenders and borrowers. Hence, our paper is the first one that incorporate both lenders (potential claimants) and borrowers into a bankruptcy problem.

\subsection{Road Map}

The organization of the paper is as follows: We first introduce the standard bankruptcy problem and the bankruptcy rules that we employ in this paper and provide some preparatory results in Section 2. In Section 3, we introduce the strategic model of bankruptcy under uncertainty and the bankruptcy problem with an endogenous estate. In Section 4 and its subsections, we analyze the equilibria of the bankruptcy game introduced in Section 3. Section 5 provides two extension results. Section 6 contains comparative static analyses on risk-return and income distribution parameters. In Section 7, we discuss our assumptions, results, and future research on the topic. Finally, Section 8 concludes.

\section{Bankruptcy Problems and Rules}

Bankruptcy is typically defined as a situation in which the total claims of claimants exceed the size of the available estate. It is sometimes also referred to as the conflicting claims problem.

\footnotetext{
${ }^{4}$ In fact, in our model, bankruptcy might not occur. It is the possibility of bankruptcy on which company's and agents' decisions are based.
} 
Formally, a bankruptcy problem is represented by a set of claimants $N=\{1,2, \ldots, n\}$, a claims vector $C=\left(c_{1}, c_{2}, \ldots, c_{n}\right)$ and for all $i \in N, c_{i} \in \mathbb{R}_{++}$, an estate $E \in \mathbb{R}_{++}$to be divided among the claimants and the inequality $\sum_{i \in N} c_{i}>E$. We denote the set of all such bankruptcy problems $(C, E)$ by $\mathcal{B}$.

A bankruptcy rule is a mechanism that allocates the estate to claimants given any bankruptcy problem. Formally, a bankruptcy rule $F$ is a function mapping each bankruptcy problem $(C, E) \in \mathcal{B}$ into $\mathbb{R}_{+}^{n}$ such that for all $i \in N, F_{i}(C, E) \in\left[0, c_{i}\right]$ and $\sum_{i \in N} F_{i}(C, E)=E$. Below, we define the bankruptcy rules we use in our base model.

The proportional rule allocates the estate proportionally with respect to claims.

Definition 1 (Proportional Rule) For all $(C, E) \in \mathcal{B}$, we have $P(C, E) \equiv \lambda_{p} C$, where $\lambda_{p}$ is given by $\lambda_{p}=\left(E / \sum_{i \in N} c_{i}\right)$.

The constrained equal awards rule allocates the estate as equal as possible taking claims as upper bounds.

Definition 2 (Constrained Equal Awards Rule) For all $(C, E) \in \mathcal{B}$, and all $j \in N$, we have $C E A_{j}(C, E) \equiv \min \left\{c_{j}, \lambda_{\text {cea }}\right\}$, where $\lambda_{\text {cea }}$ solves $\sum_{i \in N} \min \left\{c_{i}, \lambda_{\text {cea }}\right\}=E$.

The constrained equal losses rule allocates the shortage of the estate (i.e., the total loss due to bankruptcy) in an equal way (shares bounded below by zero).

Definition 3 (Constrained Equal Losses Rule) For all $(C, E) \in \mathcal{B}$, and all $j \in N$, we have $C E L_{j}(C, E) \equiv \max \left\{0, c_{j}-\lambda_{\text {cel }}\right\}$, where $\lambda_{\text {cel }}$ solves $\sum_{i \in N} \max \left\{0, c_{i}-\lambda_{\text {cel }}\right\}=E$.

Example $1 N=\{1,2,3,4,5\}, C=(10,30,40,70,100)$ and $E=180$.

\begin{tabular}{c|ccccc} 
agent & 1 & 2 & 3 & 4 & 5 \\
\hline claim & 10 & 30 & 40 & 70 & 100 \\
$P$ & 7.2 & 21.6 & 28.8 & 50.4 & 72 \\
$C E A$ & 10 & 30 & 40 & 50 & 50 \\
$C E L$ & 0 & 15 & 25 & 55 & 85
\end{tabular}

Note that under the constrained equal awards rule, claimants 1,2 , and 3 receive strictly more than what they would receive under the proportional rule, whereas claimants 4 and 5 receive strictly less than what they would receive under the proportional rule. Loosely speaking, the constrained equal awards rule favors small claimants (i.e., it makes transfers from bigger claimants to smaller claimants). Also note that under the constrained equal losses rule, claimants 4 and 5 receive strictly more than what they would receive under the proportional rule whereas claimants 1,2 , and 3 receive strictly less than what they would receive under the proportional rule. Loosely speaking, the constrained equal losses rule favors big claimants (i.e., it makes transfers from smaller claimants to bigger claimants). Later, we will make use of these facts in our analysis.

Below we prove a lemma that formalizes the idea of inter-claimant transfers under the constrained equal awards and the constrained equal losses rules taking the proportional rule payoffs as basis. 
Lemma 1 Let $(C, E) \in \mathcal{B}$. Assume without loss of generality that $c_{1} \leq c_{2} \leq \ldots \leq c_{n}$. Then,

(i) there exists a critical level of claims, $c^{*}$ such that for all $i \in N$ with $c_{i}\left\langle c^{*}, C E A_{i}(C, E)\right\rangle$ $P_{i}(C, E)$ and for all $i \in N$ with $c_{i} \geq c^{*}, C E A_{i}(C, E) \leq P_{i}(C, E)$ and,

(ii) there exists a critical level of claims, $\widetilde{c}$ such that for all $i \in N$ with $c_{i}<\widetilde{c}, C E L_{i}(C, E)<$ $P_{i}(C, E)$ and for all $i \in N$ with $c_{i} \geq \widetilde{c}, C E L_{i}(C, E) \geq P_{i}(C, E)$.

Proof. See Appendix B.

In Lemma 1, for a bankruptcy rule $F \in\{C E A, C E L\}$ and an agent $i \in N$, we provided some results on $F_{i}(C, E)-P_{i}(C, E)$. From now on, for all $F \in\{P, C E A, C E L\}$, we will denote the transfer from agent $i$ to other agents (taking the proportional payoff vector as the reference point) under rule $F$ in the bankruptcy problem $(C, E)$ by $S_{i}(C, E, F)$. Hence, $S_{i}(C, E, F)=F_{i}(C, E)-P_{i}(C, E)$. The following lemma provides closed form expressions for transfers under $C E A$ and $C E L$ in the model with two types of agents (see Section 3), where agents' types refer to their claims. In the model, we denote the transfer of agent $i$ of type $t$ by $S_{t}(C, E, F)$ since, by the definitions of all bankruptcy rules $F \in\{P, C E A, C E L\}$, transfers will be equal to $S_{t}(C, E, F)$, for all agents $i$ of type $t$.

Lemma 2 Let $(C, E) \in \mathcal{B}$ and let $N_{h}\left(N_{l}\right)$ denote the non-empty set of claimants each with a claim $c_{h}\left(c_{l}\right)$, with cardinality $n_{h}\left(n_{l}\right)$. Assume that $0<c_{l}<c_{h}$. Denote the set of all claimants by $N$ with cardinality $n=n_{h}+n_{l}$. Then the following statements are valid.

(a-1) If $c_{h}>c_{l}>\lambda_{\text {cea }}$, then

(a-1-1) $S_{l}(C, E, C E A)=\frac{n_{h} E\left[c_{h}-c_{l}\right]}{\left[n_{h}+n_{l}\right]\left[n_{h} c_{h}+n_{l} c_{l}\right]}>0$ and

(a-1-2) $S_{h}(C, E, C E A)=\frac{n_{l} E\left[c_{l}-c_{h}\right]}{\left[n_{h}+n_{l}\right]\left[n_{h} c_{h}+n_{l} c_{l}\right]}<0$.

(a-2) If $c_{h}>\lambda_{\text {cea }} \geq c_{l}$, then

(a-2-1) $S_{l}(C, E, C E A)=\frac{c_{l}\left[n_{h} c_{h}+n_{l} c_{l}-E\right]}{\left[n_{h} c_{h}+n_{l} c_{l}\right]}>0$ and

(a-2-2) $S_{h}(C, E, C E A)=\frac{n_{l} c_{l}\left[E-n_{h} c_{h}-n_{l} c_{l}\right]}{n_{h}\left[n_{h} c_{h}+n_{l} c_{l}\right]}<0$.

(b-1) If $c_{h}>c_{l}>\lambda_{c e l}$, then

(b-1-1) $S_{l}(C, E, C E L)=\frac{n_{h}\left[c_{l}-c_{h}\right]\left[n_{h} c_{h}+n_{l} c_{l}-E\right]}{\left[n_{h}+n_{l}\right]\left[n_{h} c_{h}+n_{l} c_{l}\right]}<0$ and

(b-1-2) $S_{h}(C, E, C E L)=\frac{n_{l}\left[c_{h}-c_{l}\right]\left[n_{h} c_{h}+n_{l} c_{l}-E\right]}{\left[n_{h}+n_{l}\right]\left[n_{h} c_{h}+n_{l} c_{l}\right]}>0$.

(b-2) If $c_{h}>\lambda_{c e l} \geq c_{l}$, then

(b-2-1) $S_{l}(C, E, C E L)=-\frac{E}{n_{h} c_{h}+n_{l} c_{l}} c_{l}<0$ and

(b-2-2) $S_{h}(C, E, C E L)=\frac{n_{l}}{n_{h}} \frac{E c_{l}}{\left[n_{h} c_{h}+n_{l} c_{l}\right]}>0$. 


\section{Proof. See Appendix B.}

Note that for all $F \in\{P, C E A, C E L\}, n_{h} S_{h}(C, E, F)+n_{l} S_{l}(C, E, F)=0$. Hence, the transfers are balanced. This is implied by the efficiency property embedded in the definition of a bankruptcy rule. Note that the reason why we state the results in Lemma 2 for two types is because we have two types of agents in our base model. Results in Lemma 2 can be generalized to any finite number of types. Below, we introduce our base model of bankruptcy with an endogeonus estate.

\section{A Strategic Model of Bankruptcy with an Endogenous Estate}

There are $n_{h}$ agents each with income $w_{h}$ and $n_{l}$ agents each with income $w_{l}$, such that $0<$ $w_{l}<w_{h} .{ }^{5}$ Accordingly, $N_{h}$ is the set of type $h$ agents with $\left|N_{h}\right|=n_{h}$ and $N_{l}$ is the set of type $l$ agents with $\left|N_{l}\right|=n_{l}$. We use $t$ to refer to a generic type i.e., $t \in\{l, h\}$. Therefore, for all agents $i \in N_{l} \cup N_{h}$, individual income $w_{i} \in\left\{w_{l}, w_{h}\right\}$. Both types of agents are risk-neutral. Hence, each agent wants to choose the investment alternative that brings the maximum expected return. There are two investment alternatives: agents either invest in a company and become shareholders or deposit their money into a savings account in a bank. The company runs a risky investment project and depositing money into a bank, on the other hand, brings a risk-free return. The state space for the outcome of the risky investment project is $\Omega=\{s, f\}$ where $s$ represents success and $f$ represents failure. Hence, the outcome of the project is a random variable $\omega$. With probability $\operatorname{Pr}(\omega=s)=\pi_{s}<1$, the investment project is successful and brings a payoff of $0<r_{s} \leq 1$ to the company; with probability $\operatorname{Pr}(\omega=f)=1-\pi_{s}$, the investment project fails and brings a payoff of $r_{f}<r_{s} \leq 1$ to the company. The company promises to pay $r$ to the depositors, which satisfies $0 \leq r_{f}<r \leq r_{s} \leq 1 .^{6}$ However, if the project fails it cannot honor all claims since $r_{f}<r$. On the other hand, the savings account at the bank pays a constant return $\bar{r}{ }^{7}$ We eliminate two cases that would lead to trivial results: $r_{f}>\bar{r}$ and $\bar{r}>r$. If $r_{f}>\bar{r}$ was the case, then no agent would prefer to deposit their money to the bank and if $\bar{r}>r$ was the case, then no agent would prefer to invest in the company. Hence, to make the problem "interesting", we assume that $r_{f}<\bar{r}<r$. Thus, the risky asset offers a higher return in the case of success, but a lower return in the case of failure (bankruptcy). ${ }^{8}$ Having introduced the necessary parameters, now we define the particular class of bankruptcy problems we analyze.

Definition 4 A bankruptcy problem with an endogenous estate is a pair $(C, E)$, where $C$ is a claims vector with entries $c_{i}=(1+r) w_{i}$ for all $i \in N$ and $E=\left(1+r_{f}\right) \sum_{i \in N} w_{i}$ is the estate. The class of bankruptcy problems with an endogenous estate is denoted by $\widetilde{\mathcal{B}}$.

The endogeneity is due to the fact that the claims vector and the estate are determined by agents' decisions. Moreover, note that $C$ and $E$ in the definition of the bankruptcy problem are derived

\footnotetext{
${ }^{5}$ In fact, what we mean by $w_{t}$ is the part of the income that is reserved for investment by a type $t$ agent.

${ }^{6} r$ is not determined as a result of an optimization problem in our base model. Nevertheless, later we endogenize $r$ by incorporating it into company's optimization problem.

${ }^{7}$ An asset with a variable return and a lower risk compared to the investment in the company would also do. Results in the paper are valid with such an asset. We assumed a risk-free asset for expositional simplicity.

${ }^{8}$ We will use investing in the risky asset versus investing in the company interchangeably; and investing in the risk-free asset versus depositing money into the bank interchangeably.
} 
from $w, r_{f}$, and $r$. Hence, the data of the problem can be written as $\left(w, r_{f}, r\right)$ instead of $(C, E)$. However, to keep the exposition similar to the one in standard bankruptcy problems, we keep the $(C, E)$ notation. Our analysis will focus on the class of bankruptcy problems with an endogenous estate, unless otherwise stated. All parameters mentioned above are common knowledge. Obviously, bankruptcy is a possible event only for the first investment alternative.

The company, $m$, is an important player. It chooses a bankruptcy rule $F$, which will be implemented in case of bankruptcy. The company's objective is to maximize the investment attracted. Note that, given $r, r_{s}, r_{f}, \bar{r}$ and $\pi_{s}$, maximizing the investment volume is identical to maximizing the profit. The bankruptcy rule chosen affects agents' investment decisions since it affects their return in case of a bankruptcy. Hence, the company takes into account the possible actions of agents while choosing the bankruptcy rule. As mentioned before, we use the proportional rule, the constrained equal awards rule, and the constrained equal losses rule as benchmarks. Accordingly, the company's strategy space is denoted by $\psi_{m}=\{P, C E A, C E L\}$. The company's decision is observed by all agents. Hence, each decision of the company starts a proper subgame to be played by agents. We denote these three subgames by $\Gamma^{P}, \Gamma^{C E A}$, and $\Gamma^{C E L}$.

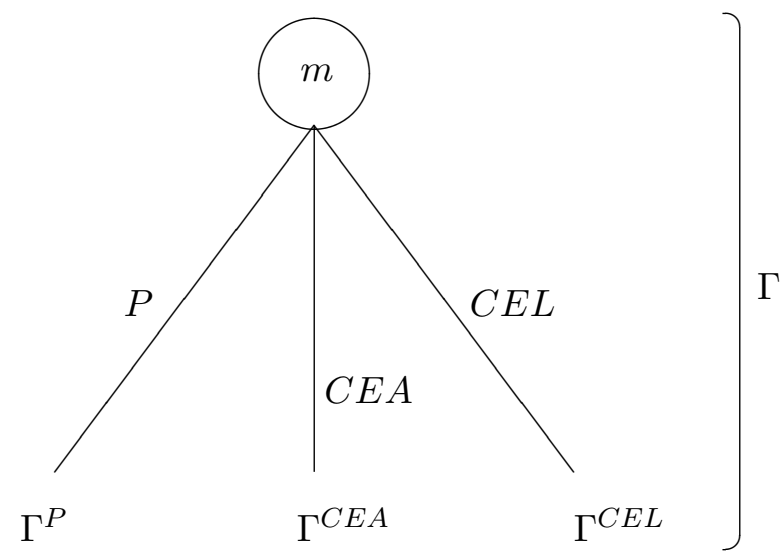

Figure 1: Game Tree

Knowing which bankruptcy rule $F$ is chosen by $m$, in the subgame $\Gamma^{F}$, all agents $i \in N_{l} \cup N_{h}$ choose whether to invest their money in the risky asset (i.e., playing $i n$ ) or to invest in the riskfree, outside asset (i.e., playing out). This decision is made by all agents simultaneously. For all $i \in N_{l} \cup N_{h}$, we denote agent $i$ 's actions by $a_{i} \in\{$ in, out $\}$ and the actions taken by agent $i$ under rule $F \in\{P, C E A, C E L\}$ as $a_{i, F}$. We describe what each agent $i$ would do in each subgame $\Gamma^{F}$ by agent $i$ 's strategy, which is denoted by $s_{i} \in \psi_{i}$. Agent $i$ 's strategy space, $\psi_{i}$, can be written as

$$
\psi_{i}=\left\{\left(a_{i, P}, a_{i, C E A}, a_{i, C E L}\right) \mid a_{i, F} \in\{\text { in }, o u t\} \text { and } F \in\{P, C E A, C E L\}\right\} .
$$

The company's payoff function is linear and we denote it as $V_{m}(F)=\sum_{t \in\{l, h\}} n_{t, i n}(F) w_{t}$, where $n_{t, i n}(F)$ stands for the number of type $t$ agents who play in in the subgame $\Gamma^{F}$. Therefore, we can write the company's objective to maximize the investment as 


$$
\max _{F \in\{P, C E A, C E L\}} V_{m}(F) .
$$

Note that once $r$ and $r_{f}$ are fixed, the estate $E$ and the claims vector $C$ are both determined by agents' actions. Since agent $i$ 's payoff under bankruptcy is determined by $F, n_{h, \text { in }}$ and $n_{l, i n}$, when writing agents' payoffs under bankruptcy, we employ the notation, $V_{i, i n}\left(F, n_{h, i n}, n_{l, i n}\right)$. Similarly, agent $i$ 's transfer under rule $F$ can be written as $S_{i}\left(F, n_{h, i n}, n_{l, i n}\right)$.

Now, given agent $i$ 's action in $\Gamma^{F}$, the payoff of agent $i \in N_{l} \cup N_{h}$ can be written as

$$
V_{i}\left(F, a_{i, F}\right)=\left\{\begin{aligned}
V_{i, \text { out }}= & (1+\bar{r}) w_{i}, \text { if } a_{i, F}=\text { out } \\
V_{i, \text { in }}^{e}\left(F, n_{h, \text { in }}, n_{l, \text { in }}\right)= & \pi_{s}(1+r) w_{i}+\left(1-\pi_{s}\right)\left[V_{i, \text { in }}\left(P, n_{h, \text { in }}, n_{l, \text { in }}\right)\right. \\
& \left.+S_{i}\left(F, n_{h, \text { in }}, n_{l, \text { in }}\right)\right], \text { if } a_{i, F}=\text { in }
\end{aligned}\right.
$$

where the superscript $e$ refers to the expected value. ${ }^{9}$ Notice that the first part of $V_{i, i n}^{e}\left(F, n_{h, i n}, n_{l, i n}\right)$ is agent $i$ 's payoff in case of successful completion of the project and the second part is his payoff in case of a bankruptcy. Also note that for the proportional rule, for all $i \in N_{l} \cup N_{h}, S_{i}\left(P, n_{h, i n}, n_{l, i n}\right)=$ 0 .

The following lemma enables us to simplify the notation $V_{j, i n}\left(P, n_{h, i n}, n_{l, i n}\right)$, since it shows that the payoff each agent receives under $P$ is independent from other agents' types, actions, etc. Consequently, we can write the payoff of agent $j$ under the proportional rule as $P_{j}$.

Lemma 3 Assume that for all $j \in N_{l} \cup N_{h}$, the claim structure is $c_{j}=(1+r) w_{j}$ and the estate is $E=\left(1+r_{f}\right) \sum_{i \in N_{l} \cup N_{h}} w_{i}$. Then $V_{j, i n}\left(P, n_{h, i n}, n_{l, i n}\right) \equiv P_{j}=\left(1+r_{f}\right) w_{j}$.

Proof. See Appendix B.

The result above is valid for any finite number of types. By Lemma 3 , if agent $i$ is of type $t$, then $V_{i, i n}\left(P, n_{h, i n}, n_{l, i n}\right)=\left(1+r_{f}\right) w_{t}$. We rewrite agent $i$ 's expected payoff under $P$ as

$$
P_{i}^{e}=\pi_{s}(1+r) w_{i}+\left(1-\pi_{s}\right) P_{i} .
$$

Using the expected payoff under the proportional rule, we can rewrite agent $i$ 's expected payoff under $C E A$ as

$$
\begin{aligned}
V_{i, i n}^{e}\left(C E A, n_{h, i n}, n_{l, i n}\right) & =\pi_{s}(1+r) w_{i}+\left(1-\pi_{s}\right)\left[P_{i}+S_{i}\left(C E A, n_{h, i n}, n_{l, i n}\right)\right] \\
& =P_{i}^{e}+\left(1-\pi_{s}\right) S_{i}\left(C E A, n_{h, i n}, n_{l, i n}\right),
\end{aligned}
$$

where $P_{i}^{e}$ stands for the expected payoff that agent $i$ would get under the proportional rule and $S_{i}\left(C E A, n_{h, i n}, n_{l, i n}\right)$ is the transfer that agent $i$ makes/receives under $C E A$ when $n_{h, i n}$ type $h$ agents and $n_{l, i n}$ type $l$ agents play $i n$. Similarly, under $C E L$, the expected payoff of agent $i$ can be rewritten as

$$
\begin{aligned}
V_{i, i n}^{e}\left(C E L, n_{h, i n}, n_{l, i n}\right) & =\pi_{s}(1+r) w_{i}+\left(1-\pi_{s}\right)\left[P_{i}+S_{i}\left(C E L, n_{h, i n}, n_{l, i n}\right)\right] \\
& =P_{i}^{e}+\left(1-\pi_{s}\right) S_{i}\left(C E L, n_{h, i n}, n_{l, i n}\right),
\end{aligned}
$$

\footnotetext{
${ }^{9}$ As one can see in (3), in defining agents' payoffs from playing in in case of bankruptcy, we use the proportional rule payoff as a benchmark.
} 
where $S_{i}\left(C E L, n_{h, i n}, n_{l, i n}\right)$ is the transfer that agent $i$ makes/receives under $C E L$ when $n_{h, i n}$ type $h$ agents and $n_{l, i n}$ type $l$ agents play $i n$.

Table 1 below, together with the sequence of actions described before and summarized in Figure 1 , defines the sequential game $\Gamma$ with three proper subgames $\Gamma^{P}, \Gamma^{C E A}$, and $\Gamma^{C E L}$ :

\begin{tabular}{||c|c||}
\hline \hline Players & $\{m\} \cup N_{l} \cup N_{h}$ \\
\hline Strategies & $\psi_{m} \times \prod_{i \in N_{l} \cup N_{h}} \psi_{i}$ \\
\hline Payoffs & $\left(V_{m}(F),\left(V_{i, s_{i}}\left(F, s_{-i}\right)\right), i \in N_{l} \cup N_{h}\right.$ \\
\hline
\end{tabular}

Table 1: Sequential Game $\Gamma$

where $s_{-i}$ denotes all agents' strategies except agent $i$. We look for pure strategy equilibria of this game. Since we want to capture sequential rationality, the equilibrium concept that we employ is that of Subgame Perfect Nash Equilibrium.

\section{Analysis of Equilibrium Decisions}

Now, we analyze the equilibria of the game defined above. We start the analysis in a backward induction fashion with the subgames $\Gamma^{F} \in\left\{\Gamma^{P}, \Gamma^{C E A}, \Gamma^{C E L}\right\}$ played among all agents $i \in N_{l} \cup N_{h}$. Therefore, in the following, when we use the term "equilibrium", it refers to the agents' equilibrium actions in the corresponding subgame. After analyzing agents' behavior in each subgame, we analyze the company's action in equilibrium. This is followed by the description of all equilibria of the game along with the resulting investment in the company.

Before the analysis of agents' investment decisions in equilibrium, we prove some preparatory lemmas and corollaries. The following corollary provides values of $c^{*}$ and $\widetilde{c}$ in the class of bankruptcy problems with endogenous estates. The following result provides closed form expressions for $c^{*}$ and $\widetilde{c}$ in this class. It follows from the proof of Lemma 1. Recall that for all $i \in N, w_{i} \in\left\{w_{l}, w_{h}\right\}$, where $0<w_{l}<w_{h}$ and $|N|=n=n_{h}+n_{l}$. Moreover, $n_{\text {in }}$ refers to total number of agents who invest in the company.

Corollary 1 Let $(C, E) \in \widetilde{\mathcal{B}}$. Then,

(i) $c^{*}=\frac{n_{h, i n}}{n_{i n}-n_{l, i n}}(1+r) w_{h}-\frac{n_{l, i n}}{n_{i n}-n_{l, i n}} \frac{(1+r)\left(r-r_{f}\right)}{\left(1+r_{f}\right)} w_{l}$ and

(ii) $\widetilde{c}=\frac{n_{h, i n}}{n_{i n}-n_{l, i n}}(1+r) w_{h}-\frac{n_{l, i n}}{n_{i n}-n_{l, i n}} \frac{(1+r)\left(1+r_{f}\right)}{\left(r-r_{f}\right)} w_{l}$.

Proof. Recall formulas (14) and (15) from the proof of Lemma 1. If we plug into (14) and (15)

$$
\begin{aligned}
\left(1+r_{f}\right)\left(n_{h, i n} w_{h}+n_{l, i n} w_{l}\right) & \text { for } E, \\
(1+r) w_{i} & \text { for } c_{i}, \text { and } \\
(1+r)\left(n_{h, i n} w_{h}+n_{l, i n} w_{l}\right) & \text { for } \sum_{i \in N} c_{i},
\end{aligned}
$$

then we obtain the equations in (i) and (ii).

The following corollary of Lemma 2 derives closed form functions of transfers under $C E A$ and $C E L$. 
Corollary 2 Let $(C, E) \in \widetilde{\mathcal{B}}$.

(a-1) If $c_{h}>c_{l}>\lambda_{c e a}$, then

(a-1-1) $S_{l}\left(C E A, n_{h, \text { in }}, n_{l, i n}\right)=\frac{n_{h, \text { in }}}{n_{h, \text { in }}+n_{l, i n}}\left[\left(1+r_{f}\right)\left(w_{h}-w_{l}\right)\right] \geq 0$ and

(a-1-2) $S_{h}\left(C E A, n_{h, i n}, n_{l, i n}\right)=\frac{n_{l, i n}}{n_{h, \text { in }}+n_{l, i n}}\left[\left(1+r_{f}\right)\left(w_{l}-w_{h}\right)\right] \leq 0$.

(a-2) If $c_{h}>\lambda_{\text {cea }} \geq c_{l}$, then

(a-2-1) $S_{l}\left(C E A, n_{h, i n}, n_{l, i n}\right)=\left(r-r_{f}\right) w_{l}>0$ and

(a-2-2) $S_{h}\left(C E A, n_{h, i n}, n_{l, i n}\right)=\frac{n_{l, i n}}{n_{h, i n}}\left(r_{f}-r\right) w_{l} \leq 0$.

(b-1) If $c_{h}>c_{l}>\lambda_{c e l}$, then

(b-1-1) $S_{l}\left(C E L, n_{h, i n}, n_{l, i n}\right)=\frac{n_{h, \text { in }}}{n_{h, \text { in }}+n_{l, i n}}\left[\left(r-r_{f}\right)\left(w_{l}-w_{h}\right)\right] \leq 0$ and

(b-1-2) $S_{h}\left(C E L, n_{h, i n}, n_{l, i n}\right)=\frac{n_{l, i n}}{n_{h, \text { in }}+n_{l, \text { in }}}\left[\left(r-r_{f}\right)\left(w_{h}-w_{l}\right)\right] \geq 0$.

(b-2) If $c_{h}>\lambda_{\text {cel }} \geq c_{l}$, then

(b-2-1) $S_{l}\left(C E L, n_{h, i n}, n_{l, i n}\right)=-\left(1+r_{f}\right) w_{l}<0$ and

(b-2-2) $S_{h}\left(C E L, n_{h, i n}, n_{l, i n}\right)=\frac{n_{l, i n}}{n_{h, i n}}\left(1+r_{f}\right) w_{l} \geq 0$.

Proof. Recall the formulas we derived in the proof of Lemma 2. If we plug into those formulas for all $t \in\{l, h\}$,

$$
\begin{aligned}
(1+r) w_{t} & \text { for } c_{t}, \\
n_{t, i n} & \text { for } n_{t}, \text { and } \\
\left(1+r_{f}\right)\left[n_{l, i n} w_{l}+n_{h, i n} w_{h}\right] & \text { for } E,
\end{aligned}
$$

then we obtain the result.

The following lemma shows that $c^{*}$ and $\widetilde{c}$ are always between $c_{l}$ and $c_{h}$.

Lemma 4 Let $(C, E) \in \widetilde{\mathcal{B}}$. Assume that $n_{h, \text { in }}>0$ and $n_{l, \text { in }}>0$. Then, for all $n_{h, \text { in }}$ and $n_{l, i n}$, (i) $c_{l} \leq c^{*} \leq c_{h}$ and (ii) $c_{l} \leq \widetilde{c} \leq c_{h}$.

Proof. See Appendix B.

Since if one type is making transfers the other type should be receiving transfers in the case with two types, the result mentioned in Lemma 4 is intuitive. This result is required for the comparative static analyses we conduct in the following lemma. It ensures that when the number of type $t \in\{l, h\}$ agents changes, the identity of the type making transfers and the identity of the type receiving transfers stays the same.

Lemma 5 Let $(C, E) \in \widetilde{\mathcal{B}}$. Then,

(i) an increase in $n_{l, \text { in }}$ weakly decreases $S_{l}\left(C E A, n_{h, \text { in }}, n_{l, i n}\right)$, 
(ii) an increase in $n_{l, i n}$ strictly increases $S_{h}\left(C E A, n_{h, i n}, n_{l, i n}\right)$,

(iii) an increase in $n_{h, \text { in }}$ weakly increases $S_{l}\left(C E L, n_{h, i n}, n_{l, i n}\right)$,

(iv) an increase in $n_{h, \text { in }}$ strictly decreases $S_{h}\left(C E L, n_{h, i n}, n_{l, i n}\right)$,

(v) an increase in $n_{h, \text { in }}$ weakly increases $S_{l}\left(C E A, n_{h, i n}, n_{l, i n}\right)$,

(vi) an increase in $n_{h, \text { in }}$ strictly decreases $S_{h}\left(C E A, n_{h, i n}, n_{l, i n}\right)$,

(vii) an increase in $n_{l, \text { in }}$ weakly decreases $S_{l}\left(C E L, n_{h, i n}, n_{l, i n}\right)$, and

(viii) an increase in $n_{l, \text { in }}$ strictly increases $S_{h}\left(C E L, n_{h, i n}, n_{l, i n}\right)$.

Proof. See Appendix B.

The comparative statics stated in Lemma 5 have simple intuitions. In a nutshell, they show the changes in per-capita transfer with respect to changes in the number of type $h$ and type $l$ agents. We see that if the number of agents of types who are making transfers increases, per-capita transfers they make decrease and per-capita transfers other types receive increase. On the other hand, if the number of agents of types who are receiving transfers increases, per-capita transfers they receive decrease and per-capita transfers other types make increase. The following lemma states that under $P$, a type $l$ agent prefers to play in if and only if a type $h$ agent prefers to play in.

Lemma 6 Given a bankruptcy problem $(C, E) \in \widetilde{\mathcal{B}}, P_{h} \geq V_{h}$ if and only if $P_{l} \geq V_{l}$.

Proof. See Appendix B.

Tie-Breaking Assumption Every agent plays in when he is indifferent between in and out.

This tie-breaking assumption is employed in the rest of the paper. The following lemma states that each agent's decision in equilibrium is determined by his type only.

Lemma 7 (Symmetry) If agents $i$ and $j$ are of the same type $t \in\{l, h\}$, their strategies are the same in equilibrium.

Proof. See Appendix B.

Lemma 7 has three important implications. First of all, it shows that if there exists an equilibrium it will be symmetric, i.e., same types play the same strategy in equilibrium. The tie-breaking assumption is important for the validity of Lemma 7. If agents of the same type play strategies that are different from each other when these agents are indifferent, the statement in Lemma 7 is not valid anymore. However, breaking the ties in favor of playing in is not crucial for the proofs. Assuming that every agent plays out when he is indifferent would work equally well.

Second, this symmetry result enables us to employ a more compact notation for equilibrium actions in subgames $\Gamma^{F}$ (in game $\Gamma$ ): $\left(a_{h, F}, a_{l, F}\right)$ means that all type $h$ agents play $a_{h, F}$ and all type $l$ agents play $a_{l, F}$ in the subgame $\Gamma^{F}$. 
Third, this result also enables us to use a simpler notation when writing agents' expected payoffs. Since we know that agents of the same type act identically, we can write the expected payoff of a representative type $t$ agent who plays in under rule $F$ as $V_{t}^{e}\left(F, s_{-t}\right)$ instead of writing individual expected payoff as $V_{i, i n}^{e}\left(F, n_{h, i n}, n_{l, i n}\right) .{ }^{10}$ We will employ this notation in the remaining part of the model.

The following corollary relates the symmetry result to the equilibrium values of $V_{m}(F)$. Since we show that agents of the same type have the same strategies in equilibrium, this reduces the number of possible values of equilibrium investment volume.

Corollary 3 In equilibrium, $V_{m}(F)$ can take only four values: $0, n_{h} w_{h}, n_{l} w_{l}$ and $n_{h} w_{h}+n_{l} w_{l}$.

Proof. Since there are two types of agents, by Lemma 7, there are four possible combinations of strategy profiles under the symmetry result proven above:

(i) Both types play out. Thus, $V_{m}(F)=0$,

(ii) Type $h$ agents play in, type $l$ agents play out. Thus, $V_{m}(F)=n_{h} w_{h}$,

(iii) Type $h$ agents play out, type $l$ agents play $i n$. Thus, $V_{m}(F)=n_{l} w_{l}$,

(iv) Both types play in. Thus, $V_{m}(F)=n_{h} w_{h}+n_{l} w_{l}$.

The following lemma shows that certain strategy profiles cannot exist in any equilibrium under $C E A$ and $C E L$.

Lemma 8 The following statements about strategy profiles are valid.

(i) In the subgame $\Gamma^{C E A}$, the strategy profile (for all $i \in N_{h}, s_{i}=\left(.\right.$, in,.) and for all $j \in N_{l}$, $s_{j}=(.$, out,.$)$ cannot be an equilibrium,

(ii) In the subgame $\Gamma^{C E L}$, the strategy profile (for all $i \in N_{h}, s_{i}=\left(., .\right.$, out) and for all $j \in N_{l}$, $s_{j}=(., .$, in $)$ cannot be an equilibrium.

Proof. See Appendix B.

The result in this lemma has a simple intuition: if, in equilibrium, the parameter values are such that even the type of agents who are making transfers find playing in optimal, the type of agents who are receiving transfers also find it optimal to play $i n$.

\subsection{Characterization of All Nash Equilibria in $\Gamma^{P}, \Gamma^{C E A}$, and $\Gamma^{C E L}$}

In this subsection, we describe agents' investment behavior and characterize all Nash equilibria in subgames $\Gamma^{P}, \Gamma^{C E A}$, and $\Gamma^{C E L}$. Recall that Lemma 7 enables us to use type best responses instead of agent best responses. Hence, in this section, we use type t's best response to a strategy played by the other type. Denote the best response of type $t$ agents to action $a_{-t}$ played by the other type of agents in the subgame $\Gamma^{F}$ by $B R_{t}\left(F, a_{-t}\right){ }^{11}$

\footnotetext{
${ }^{10}$ The equal treatment of equals property asserts that the agents with equal claims should receive the same payoff. This property is satisfied by all rules we consider here, which enables us to use this compact notation.

${ }^{11}$ Also note that each agent has one information set in each subgame and two actions. Therefore, the terms strategy and action refer to same objects in subgames $\Gamma^{P}, \Gamma^{C E A}$, and $\Gamma^{C E L}$. Hence, we prefer to stick to action notation instead of introducing strategy notation for subgames, although we use the terms strategy and action interchangeably.
} 


\section{Under the Proportional Rule:}

In the subgame $\Gamma^{P}$, the following payoff matrix can be used to show representative type $h$ and type $l$ agent's expected payoffs. The first (second) item in each cell represents each type $h$ (type $l$ ) agent's expected payoff. All matrices are drawn for representative agents of type $h$ and type $l$.

\begin{tabular}{|c|c|c|}
\hline$h \backslash l$ & in & out \\
\hline in & $P_{h}^{e}, P_{l}^{e}$ & $P_{h}^{e}, V_{l, \text { out }}$ \\
\hline out & $V_{h, \text { out }}, P_{l}^{e}$ & $V_{h, \text { out }}, V_{l, \text { out }}$ \\
\hline
\end{tabular}

Table 2: Payoff Matrix under the Proportional Rule

Recall that by Lemma 3, the expected payoff of each agent is independent of other agents' strategies under $P$. This implies that all equilibria are dominant strategy equilibria. Also, remember that by Lemma $6, P_{l}^{e}<V_{l, \text { out }}$ if and only if $P_{h}^{e}<V_{h, \text { out }}$. Therefore, if $P_{h}^{e} \geq V_{h, \text { out }}$, then $B R_{h}(P$, in $)=B R_{h}(P$, out $)=i n$, and similarly if $P_{l}^{e} \geq V_{l, \text { out }}$, then $B R_{l}(P$, in $)=B R_{l}(P$, out $)=$ in. If $P_{h}^{e}<V_{h, \text { out }}$, then $B R_{h}(P$, in $)=B R_{h}(P$, out $)=$ out, and similarly if $P_{l}^{e}<V_{l, \text { out }}$, then $B R_{l}(P$, in $)=B R_{l}(P$, out $)=$ out. Also recall that $\left(a_{h, F}, a_{l, F}\right)$ means that all type $h$ agents play $a_{h, F}$ and all type $l$ agents play $a_{l, F}$ in the subgame $\Gamma^{F}$. We now describe agents' equilibrium strategies in the subgame $\Gamma^{P}$.

Equilibria in the subgame $\Gamma^{P}$ :

Case 1 If for all $t \in\{l, h\}, P_{t}^{e}<V_{t, \text { out }}$, then the unique equilibrium strategy profile is $\left(a_{h, P}, a_{l, P}\right)=$ (out, out).

Case 2 If for all $t \in\{l, h\}, P_{t}^{e} \geq V_{t, \text { out }}$, then the unique equilibrium strategy profile is $\left(a_{h, P}, a_{l, P}\right)=$ $($ in, in $)$.

Note that neither (in,out) nor (out,in) equilibria are possible. This is due to proportionality, which implies that $P_{h}^{e} \geq V_{h, o u t}$ if and only if $P_{l}^{e} \geq V_{l, o u t}$.

\section{Under the Constrained Equal Awards Rule:}

In the subgame $\Gamma^{C E A}$ :

\begin{tabular}{|c|c|c|}
\hline$h \backslash l$ & in & out \\
\hline in & $\begin{array}{c}P_{h}^{e}+\left(1-\pi_{s}\right) S_{h}(C E A, \text { in }), \\
P_{l}^{e}+\left(1-\pi_{s}\right) S_{l}(C E A, \text { in })\end{array}$ & $P_{h}^{e}, V_{l, \text { out }}$ \\
\hline out & $V_{h, \text { out }}, P_{l}^{e}$ & $V_{h, \text { out }}, V_{l, \text { out }}$ \\
\hline
\end{tabular}

Table 3: Payoff Matrix under the Constrained Equal Awards Rule 
By the definition of the constrained equal awards rule and Lemma $1, S_{h}(C E A$, in $)<0$ and $S_{l}(C E A, i n)>0$. If the outside asset pays more than the best possible expected payoff that type $l$ agents can get, the analysis is trivial since then type $l$ agents would never play $i n$. Hence, we assume that $V_{l}^{e}(C E A$, in $)=P_{l}^{e}+\left(1-\pi_{s}\right) S_{l}(C E A$, in $)>V_{l, o u t}$. This assumption implies that $B R_{l}(C E A$, in $)=i n$. The relationship between $P_{l}^{e}$ and $V_{l, \text { out }}$ determines type $l$ agents' best response to type $h$ agents playing out. If $P_{l}^{e} \geq V_{l}$, then $B R_{l}(C E A$, out $)=i n$; if $P_{l}<V_{l, \text { out }}$, then $B R_{l}(C E A$, out $)=$ out. On the other hand, type $h$ 's best response against in depends on the relationship between $V_{h}^{e}(C E A, i n)=P_{h}^{e}+\left(1-\pi_{s}\right) S_{h}(C E A$, in $)$ and $V_{h, \text { out }}$. If

$$
V_{h}^{e}(C E A, \text { in })=P_{h}^{e}+\left(1-\pi_{s}\right) S_{h}(C E A, \text { in }) \geq V_{h, \text { out }},
$$

then $B R_{h}(C E A$, in $)=i n$; if

$$
V_{h}^{e}(C E A, \text { in })=P_{h}^{e}+\left(1-\pi_{s}\right) S_{h}(C E A, \text { in })<V_{h, \text { out }},
$$

then $B R_{h}(C E A, i n)=$ out. Therefore, these inequalities characterize agents' equilibrium strategies in the subgame $\Gamma^{C E A}$.

Equilibria in the subgame $\Gamma^{C E A}$ :

Case 1 If for all $t \in\{l, h\}, P_{t}^{e}<V_{t, o u t}$, then the unique equilibrium strategy profile is $\left(a_{h, C E A}, a_{l, C E A}\right)=($ out, out $)$.

Case 2 If for all $t \in\{l, h\}, P_{t}^{e} \geq V_{t, \text { out }}$ and $V_{h}^{e}(C E A$, in $)=P_{h}^{e}+\left(1-\pi_{s}\right) S_{h}(C E A$, in $)<V_{h, \text { out }}$, then the unique equilibrium strategy profile is $\left(a_{h, C E A}, a_{l, C E A}\right)=($ out, in $)$.

Case 3 If for all $t \in\{l, h\}, P_{t}^{e} \geq V_{t, \text { out }}$ and $V_{h}^{e}(C E A$, in $)=P_{h}^{e}+\left(1-\pi_{s}\right) S_{h}(C E A$, in $) \geq V_{h, \text { out }}$, then the unique equilibrium strategy profile is $\left(a_{h, C E A}, a_{l, C E A}\right)=(i n, i n)$.

\section{Under the Constrained Equal Losses Rule:}

In the subgame $\Gamma^{C E L}$ :

\begin{tabular}{|c|c|c|}
\hline$h \backslash l$ & in & out \\
\hline \multirow{2}{*}{ in } & $\begin{array}{c}P_{h}^{e}+\left(1-\pi_{s}\right) S_{h}(C E L, \text { in }), \\
P_{l}^{e}+\left(1-\pi_{s}\right) S_{l}(C E L, \text { in })\end{array}$ & $P_{h}^{e}, V_{l, \text { out }}$ \\
\hline out & $V_{h, \text { out }}, P_{l}^{e}$ & $V_{h, \text { out }}, V_{l, \text { out }}$ \\
\hline
\end{tabular}

Table 4: Payoff Matrix under the Constrained Equal Losses Rule

By the definition of the constrained equal losses rule and Lemma $1, S_{h}(C E L$, in $)>0$ and $S_{l}(C E L, i n)<0$. If the outside asset pays more than the best possible expected payoff that type $h$ agents can get, the analysis is trivial since then type $h$ agents would never play $i n$. Hence, we assume that $V_{h}^{e}(C E L, i n)=P_{h}^{e}+\left(1-\pi_{s}\right) S_{h}(C E L, i n)>V_{h, o u t}$. This assumption implies that $B R_{h}(C E L, i n)=i n$. The relationship between $P_{h}^{e}$ and $V_{h, o u t}$ determines type $h$ agents' best response to type $l$ agents playing out. If $P_{h}^{e} \geq V_{h}$, then $B R_{h}(C E L$, out $)=i n$; if $P_{h}<V_{h, o u t}$, then 
$B R_{h}(C E L$, out $)=$ out. On the other hand, type $l$ 's best response to type $h$ agents playing in depends on the relationship between $V_{l}^{e}(C E L, i n)=P_{l}^{e}+\left(1-\pi_{s}\right) S_{l}(C E L$, in $)$ and $V_{l, o u t}$. If

$$
V_{l}^{e}(C E L, \text { in })=P_{l}^{e}+\left(1-\pi_{s}\right) S_{l}(C E L, \text { in }) \geq V_{l, o u t},
$$

then $B R_{l}(C E L$, in $)=i n$; if

$$
V_{l}^{e}(C E L, \text { in })=P_{l}^{e}+\left(1-\pi_{s}\right) S_{l}(C E L, \text { in })<V_{l, o u t},
$$

then $B R_{l}(C E L$, in $)=$ out. Therefore, these inequalities characterize agents' equilibrium actions under the constrained equal losses rule.

Equilibria in the subgame $\Gamma^{C E L}$ :

Case 1 If for all $t \in\{l, h\}, P_{t}^{e}<V_{t, o u t}$, then the unique equilibrium strategy profile is $\left(a_{h, C E L}, a_{l, C E L}\right)=($ out, out $)$.

Case 2 If for all $t \in\{l, h\}, P_{t}^{e} \geq V_{t, \text { out }}$ and $V_{l}^{e}(C E L$, in $)=P_{l}^{e}+\left(1-\pi_{s}\right) S_{l}(C E L$, in $)<V_{l, \text { out }}$, then the unique equilibrium strategy profile is $\left(a_{h, C E L}, a_{l, C E L}\right)=($ in, out $)$.

Case 3 If for all $t \in\{l, h\}, P_{t}^{e} \geq V_{t, \text { out }}$ and $V_{l}^{e}(C E L$, in $)=P_{l}^{e}+\left(1-\pi_{s}\right) S_{l}(C E L$, in $) \geq V_{l, \text { out }}$, then the unique equilibrium strategy profile is $\left(a_{h, C E L}, a_{l, C E L}\right)=($ in,$i n)$.

Note that in a Nash equilibrium of the subgame $\Gamma^{C E A}$, if type $h$ agents choose to play $i n$, type $l$ agents also choose to play $i n$. Similarly, in a Nash equilibrium of the subgame $\Gamma^{C E L}$, if type $l$ agents choose to play in, type $h$ agents also choose to play $i n$. Also note that, if the equilibrium of the subgame $\Gamma^{P}$ is the strategy profile $\left(a_{h, P}, a_{l, P}\right)=$ (out, out), then the equilibrium strategy profiles of the subgames $\Gamma^{C E A}$ and $\Gamma^{C E L}$ are also $\left(a_{h, C E A}, a_{l, C E A}\right)=\left(a_{h, C E L}, a_{l, C E L}\right)=($ out, out $)$.

\subsection{Characterization of All Subgame Perfect Nash Equilibria in $\Gamma$}

Having finished analyzing agents' behavior in all three subgames, we analyze the company's behavior and characterize all subgame perfect Nash equilibria in $\Gamma$ in this subsection. As we mentioned in Section 3, the company's payoff function is $V_{m}(F)=\sum_{t \in\{l, h\}} n_{t, i n}(F) w_{t}$ where $n_{t, i n}(F)$ is the number of type $t$ agents played in under $F$. Therefore, the company's decision depends on the equilibrium strategies of agents in each subgame and the resulting level of investment. In the previous section, we analyzed the equilibrium strategies of agents in all three subgames. Below, we list different combinations of inequalities and the subgame perfect Nash equilibrium strategy profiles along with the equilibrium investment in the company. In the strategy profile $\left(s_{m}, s_{h}, s_{l}\right)$, the first entry refers to the company's strategy (i.e., $s_{m} \in \psi_{m}$ ), second to type $h$ agents' (i.e., $s_{h} \in \psi_{h}$ ), and third to type $l$ agents' (i.e., $s_{l} \in \psi_{l}$ ). Moreover, the first entry in a representative type $t$ agent's strategy profile refer to his equilibrium action in $\Gamma^{P}$, the second to his equilibrium action in $\Gamma^{C E A}$, and the third to his equilibrium action in $\Gamma^{C E L}$. 
C1. If for all $t \in\{l, h\}$

$$
\begin{aligned}
P_{t}^{e} & \geq V_{t, \text { out }}, \\
P_{h}^{e}+\left(1-\pi_{s}\right) S_{h}(C E A, \text { in }) & <V_{h, \text { out }}, \text { and } \\
P_{l}^{e}+\left(1-\pi_{s}\right) S_{l}(C E L, \text { in }) & <V_{l, \text { out }},
\end{aligned}
$$

then given the agents' equilibrium actions in subgames $\Gamma^{P}, \Gamma^{C E A}$, and $\Gamma^{C E L}$ presented in the previous subsection, the company prefers the proportional rule and the equilibrium investment in the company is $V_{m}(F)=\sum_{t \in\{l, h\}} n_{t} w_{t}$. As we showed in the previous subsection, under these parameter restrictions, neither the constrained equal awards rule nor the constrained equal losses rule can attract all types to invest in the company, whereas the proportional rule can. Hence, the unique subgame perfect Nash equilibrium strategy profile is

$$
\left(s_{m}, s_{h}, s_{l}\right)=(P,(\text { in } ; \text { out } ; \text { in }),(\text { in } ; \text { in } ; \text { out })) .
$$

C2. If for all $t \in\{l, h\}$,

$$
\begin{aligned}
P_{t}^{e} & \geq V_{t, \text { out }}, \\
P_{h}^{e}+\left(1-\pi_{s}\right) S_{h}(C E A, \text { in }) & \geq V_{h, \text { out }}, \text { and } \\
P_{l}^{e}+\left(1-\pi_{s}\right) S_{l}(C E L, \text { in }) & <V_{l, \text { out }},
\end{aligned}
$$

then given the agents' equilibrium actions in subgames $\Gamma^{P}, \Gamma^{C E A}$, and $\Gamma^{C E L}$ presented in the previous subsection, the company prefers the proportional rule or the constrained equal awards rule to the constrained equal losses rule and the equilibrium investment in the company is $V_{m}(F)=\sum_{t \in\{l, h\}} n_{t} w_{t}$. As we showed in the previous subsection, under these parameter restrictions, both the constrained equal awards and the proportional rules can attract all types to invest in the company whereas the constrained equal losses rule can only attract $h$ types. Hence, the subgame perfect Nash equilibrium strategy profiles are

$$
\begin{aligned}
& \left(s_{m}, s_{h}, s_{l}\right)=(P,(\text { in } ; \text { in } ; \text { in }),(\text { in } ; \text { in } ; \text { out })) \text { and } \\
& \left(s_{m}, s_{h}, s_{l}\right)=(C E A,(\text { in } ; \text { in } ; \text { in }),(\text { in } ; \text { in } ; \text { out })) .
\end{aligned}
$$

C3. If for all $t \in\{l, h\}$,

$$
\begin{aligned}
P_{t}^{e} & \geq V_{t, \text { out }}, \\
P_{h}^{e}+\left(1-\pi_{s}\right) S_{h}(C E A, \text { in }) & <V_{h, \text { out }}, \text { and } \\
P_{l}^{e}+\left(1-\pi_{s}\right) S_{l}(C E L, \text { in }) & \geq V_{l, \text { out }},
\end{aligned}
$$

then given the agents' equilibrium actions in subgames $\Gamma^{P}, \Gamma^{C E A}$, and $\Gamma^{C E L}$ presented in the previous subsection, the company prefers the proportional rule or the constrained equal losses rule to the constrained equal awards rule and the equilibrium investment in the company is 
$V_{m}(F)=\sum_{t \in\{l, h\}} n_{t} w_{t}$. As we showed in the previous subsection, under these parameter restrictions, the constrained equal losses and the proportional rules can attract all types to invest in the company whereas the constrained equal awards rule can only attract $l$ types. Hence, the subgame perfect Nash equilibrium strategy profiles are

$$
\begin{aligned}
& \left(s_{m}, s_{h}, s_{l}\right)=(P,(\text { in } ; \text { out } ; \text { in }),(\text { in } ; \text { in } ; \text { in })) \text { and } \\
& \left(s_{m}, s_{h}, s_{l}\right)=(\text { CEL },(\text { in } ; \text { out } ; \text { in }),(\text { in } ; \text { in } ; \text { in })) .
\end{aligned}
$$

C4. If for all $t \in\{l, h\}$,

$$
\begin{aligned}
P_{t}^{e} & \geq V_{t, \text { out }}, \\
P_{h}^{e}+\left(1-\pi_{s}\right) S_{h}(C E A, \text { in }) & \geq V_{h, \text { out }}, \text { and } \\
P_{l}^{e}+\left(1-\pi_{s}\right) S_{l}(C E L, \text { in }) & \geq V_{l, \text { out }},
\end{aligned}
$$

then given the agents' equilibrium actions in subgames $\Gamma^{P}, \Gamma^{C E A}$, and $\Gamma^{C E L}$ presented in the previous subsection, the company is indifferent between all three rules, and the equilibrium investment in the company is $V_{m}(F)=\sum_{t \in\{l, h\}} n_{t} w_{t}$. As we showed in the previous subsection, under these parameter restrictions, all rules are equally able to attract all types to invest in the company. Hence, the subgame perfect Nash equilibrium strategy profiles are

$$
\begin{aligned}
& \left(s_{m}, s_{h}, s_{l}\right)=(P,(i n ; i n ; i n),(i n ; i n ; i n)), \\
& \left(s_{m}, s_{h}, s_{l}\right)=(C E A,(i n ; i n ; i n),(i n ; i n ; i n)), \text { and } \\
& \left(s_{m}, s_{h}, s_{l}\right)=(C E L,(i n ; i n ; i n),(i n ; i n ; i n)) .
\end{aligned}
$$

C5. If for all $t \in\{l, h\}$,

$$
P_{t}^{e}<V_{t, \text { out }},
$$

then given the agents' equilibrium actions in subgames $\Gamma^{P}, \Gamma^{C E A}$, and $\Gamma^{C E L}$ presented in the previous subsection,the company is indifferent between all three rules, and the equilibrium investment in the company is $V_{m}(F)=0$. Since $P_{t}^{e}<V_{t, \text { out }}$ is a necessary condition for both types of agents' equilibrium decisions to in none of the rules can attract neither of the two types to invest in the company. Hence, the subgame perfect Nash equilibrium strategy profiles are

$$
\begin{aligned}
& \left(s_{m}, s_{h}, s_{l}\right)=(P,(\text { out } ; \text { out } ; \text { out }),(\text { out } ; \text { out } ; \text { out })) \\
& \left(s_{m}, s_{h}, s_{l}\right)=(\text { CEA },(\text { out } ; \text { out } \text { out }),(\text { out } ; \text { out } ; \text { out })), \text { and } \\
& \left(s_{m}, s_{h}, s_{l}\right)=(\text { CEL },(\text { out } ; \text { out } ; \text { out }),(\text { out } ; \text { out } ; \text { out })) .
\end{aligned}
$$


Note that in C4 and C5 above, the company's decision does not really matter. Basically, in these cases, anything goes. As we have shown above, besides $P_{t}^{e} \geq V_{t, o u t}$,

$$
\begin{aligned}
P_{h}^{e}+\left(1-\pi_{s}\right) S_{h}(C E A, \text { in }) & \geq V_{h, \text { out }} \text { and } \\
P_{l}^{e}+\left(1-\pi_{s}\right) S_{l}(C E L, \text { in }) & \geq V_{l, \text { out }}
\end{aligned}
$$

should be satisfied in $\mathrm{C} 4$. The interpretation of this is that neither under $C E A$ nor under $C E L$, per-capita transfers from disadvantaged type of agents to advantaged type of agents are significantly high. This, intuitively, can be due to (i) a small difference between $w_{l}$ and $w_{h}$, (ii) a low probability of bankruptcy (i.e., $1-\pi_{s}$ ), or (iii) a small outside asset payoff $(\bar{r})$ in $\mathrm{C} 4$.

C5 shows another situation in which the decision will not make a difference. No matter which rule the company chooses, the investment in the company will be 0 . However, this has nothing to do with the income distribution in the society. We already showed in Lemma 6 that $P_{t}^{e} \geq$ $V_{t, o u t}$ does not contain any income distribution parameters (e.g., $n_{l}, n_{h}, w_{l}$ and $w_{h}$ ). Hence, the validity of this condition depends only on the risk-return characteristics of investment alternatives. Intuitively, if the payoff from the risk-free asset is sufficiently high, or the probability of bankruptcy is sufficiently high (or more generally the expected return from the risky investment is sufficiently low) then $P_{t}^{e}<V_{t, \text { out }}$ will hold, in which case the company's decision cannot change the equilibrium investment in the company. We analyze the effect of changes in the parameters on the equilibrium and the corresponding investment in the company in more detail in Section 6.

\subsection{Equilibrium and Results}

In Subsections 4.1 and 4.2, we analyzed agents' and the company's decisions and characterized all subgame perfect Nash equilibria. In this subsection, we present some results which are implied by this equilibrium analysis. The following proposition states that there always exists a pure strategy subgame perfect Nash equilibrium of game $\Gamma$.

Proposition 1 A pure strategy subgame perfect Nash equilibrium of $\Gamma$ exists.

Proof. Follows from the analyses in Subsections 4.1 and 4.2.

Below, we present our main result of the base model. It shows that the proportional rule has a very strong position in our strategic setting.

Theorem 1 For any bankruptcy problem $(C, E) \in \widetilde{\mathcal{B}}$, there always exists a subgame perfect Nash equilibrium of $\Gamma$, which involves the proportional rule.

Proof. Notice that in all five cases analyzed in Subsection 4.2, the subgame perfect Nash equilibria involves the proportional rule. Since, we characterized all equilibria in Subsection 4.2, the result immediately follows.

Notice that in C3, the subgame perfect Nash Equilibria do not involve the constrained equal awards rule, in $\mathrm{C} 2$, the subgame perfect Nash equilibria do not involve the constrained equal losses rule and in $\mathrm{C} 1$, the subgame perfect Nash Equilibria involve neither of these two rules. Hence, the statement in the main theorem is valid only for the proportional rule.

The following corollary shows that our main result is robust with respect to changes in the income distribution. 
Corollary 4 The statement in the main theorem is valid independent of the income distribution.

Proof. Take any income distribution characterized by the parameters, $n_{h}, n_{l}, w_{h}$ and $w_{l}$. The condition that determines equilibrium under $P$ is: for all $t \in\{l, h\}, P_{t}^{e} \geq V_{t, \text { out }}$, which is independent of the income distribution parameters as shown in Lemma 6. Thus, the result follows.

Note that $P_{h}^{e}+\left(1-\pi_{s}\right) S_{h}(C E A$, in $) \geq V_{h, \text { out }}$ and $P_{l}^{e}+\left(1-\pi_{s}\right) S_{l}(C E L$, in $) \geq V_{l, \text { out }}$ depend on income distribution parameters.

Theorem 1 and Corollary 4 provide a justification from a noncooperative point of view for the fact that the proportional rule is frequently employed in allocating a bankrupt company's assets to shareholders.

By Corollary 4, even if there is an uncertainty about the income distribution (i.e., the company does not know the income distribution for sure) statements in the Theorem 1 are still valid. In fact, for probability distributions that assign non-zero probability to all possible income distributions, the proportional rule would be the unique optimal strategy for an expected-payoff maximizing company. Also, note that by Theorem 1 and Corollary 4, the average investment in the company under the proportional rule is largest among the three rules.

Now suppose that the company chooses $r$ along with $F$ to maximize the profit and later agents decide on whether to invest in the company or invest in the risk-free asset. This endogenizes the return rate, $r$. Optimization on $r$ boils down to choosing a level of $r$ that attracts the maximum amount of investment in a cheapest way. Hence, the company chooses an $r$ that makes agents indifferent between playing in and playing out. The following corollary shows that the result in the main theorem becomes even stronger when we endogenize $r .{ }^{12}$

Corollary 5 Denote the extended game in which the company chooses $F$ and $r$ simultaneously by $\Gamma^{r, F}$. Then, for any bankruptcy problem $(C, E) \in \widetilde{\mathcal{B}}$, there exists a unique subgame perfect Nash equilibrium of $\Gamma^{r, F}$, which involves the proportional rule.

Proof. The proof follows directly from the fact that the necessary and sufficient condition(s) for agents to play in under the proportional rule is less restrictive than the corresponding conditions under other rules. Hence, there always exists an $r$ under $P$, which make agents play in and is still lower than those corresponding values of $r$ under the other rules.

\section{Extensions}

\subsection{Larger Set of Rules}

In this subsection, we show that our results remain valid if we enlarge the set of rules we use in our model. Our first candidate is the Talmud rule since it is one of the prominent bankruptcy rules, along with three rules analyzed above and satisfies a large set of "desirable" properties (see Herrero \& Villar, 2001 and Thomson, 2006). Aumann \& Maschler (1985), in a seminal article, propose the Talmud rule as a consistent extension of the contested garment rule.

\footnotetext{
${ }^{12}$ I would like to thank Kevin Hasker for pointing out this possible extension after a seminar.
} 
Definition 5 (Talmud Rule) For all $(C, E) \in \widetilde{\mathcal{B}}$, and all $j \in N$,

$$
T A L_{j}(C, E) \equiv\left\{\begin{array}{c}
\min \left\{\frac{c_{j}}{2}, \lambda_{t}\right\} \text { if } E \leq \sum_{i \in N} \frac{c_{i}}{2} \\
c_{j}-\min \left\{\frac{c_{j}}{2}, \lambda_{t}\right\} \text { if } E \geq \sum_{i \in N} \frac{c_{i}}{2}
\end{array}\right.
$$

where $\lambda_{t}$ solves $\sum_{i \in N} T A L_{i}(C, E)=E$.

Proposition 2 Denote the extended game for which $F \in\{P, C E A, C E L, T A L\}$ by $\widehat{\Gamma}$ and let $(E, C) \in \widetilde{\mathcal{B}}$,

(i) there always exists a subgame perfect Nash equilibrium of $\widehat{\Gamma}$ that involves the proportional rule, when $E \neq \sum_{i \in N} \frac{c_{i}}{2}$ and

(ii) there always exists a subgame perfect Nash equilibrium of $\widehat{\Gamma}$ that involves the proportional rule and Talmud rule, when $E=\sum_{i \in N} \frac{c_{i}}{2}$ (i.e., $r=1$ and $r_{f}=0$ ).

Proof. Note that the Talmud rule coincides with the constrained equal awards rule (on adjusted half-claims) if $E \leq \sum_{i \in N} \frac{c_{i}}{2}$ and with the constrained equal losses rule (on adjusted half-claims) if $E \geq \sum_{i \in N} \frac{c_{i}}{2}$ (see Moreno-Ternero \& Villar, 2006). When $E=\sum_{i \in N} \frac{c_{i}}{2}$, the proportional rule and the Talmud rule lead to the same awards vector. In Figure 2, path of awards for $P$ (red line), $C E A$ (green line), $C E L$ (blue line), and $T A L$ (black line) are shown.

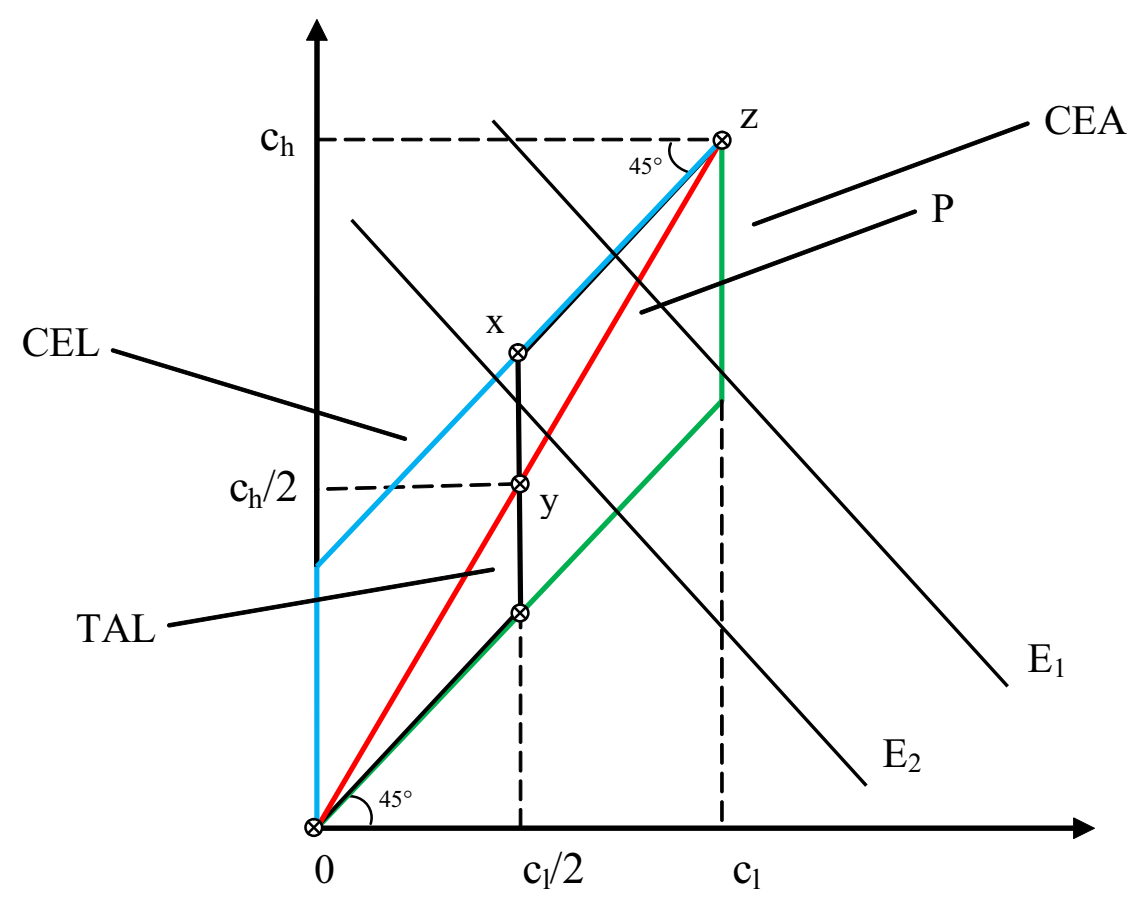

Figure 2: Path of Awards for P, CEA, CEL and, $T A L$

(i) In our model, the relative value of $E$ with respect to $\sum_{i \in N} \frac{c_{i}}{2}$ is completely determined by values of $r$ and $r_{f}$. For instance, $E<\sum_{i \in N} \frac{c_{i}}{2}$ corresponds to $1<r-2 r_{f}, E=\sum_{i \in N} \frac{c_{i}}{2}$ to $r=1$ and $r_{f}=0$, and $E>\sum_{i \in N} \frac{c_{i}}{2}$ to $r-2 r_{f}<1$. Since we do not allow $r_{f}<0$ or $r>1, E<\sum_{i \in N} \frac{c_{i}}{2}$ is not possible. Therefore, the Talmud rule coincides with the constrained equal losses rule in our model for $n_{h} c_{h}+\left(n_{l}-n_{h}\right) \frac{c_{l}}{2} \leq E \leq \sum_{i \in N} c_{i}$ (estate lines crossing the $T A L$ path of awards between 
$x$ and $z$ in Figure 2). Hence, if $n_{h} c_{h}+\left(n_{l}-n_{h}\right) \frac{c_{l}}{2} \leq E \leq \sum_{i \in N} c_{i}$, then the result in Theorem 1 holds.

(ii) On the other hand, if $\sum_{i \in N} \frac{c_{i}}{2} \leq E \leq n_{h} c_{h}+\left(n_{l}-n_{h}\right) \frac{c_{l}}{2}$ (estate lines crossing the $T A L$ path of awards between $x$ and $y$ in Figure 2), then the payoff vectors of $T A L$ and $C E L$ do not coincide. Note that the $T A L$ payoffs contain half-claims plus the $C E L$ payoffs from truncated claims (i.e., $c_{i}-\frac{c_{i}}{2}$ ) and the estate (i.e., $\left.E-\sum_{i \in N} \frac{c_{i}}{2}\right) .{ }^{13}$ Thus, per-capita transfers type $l$ agents make under $T A L$ (i.e., $S_{l}(T A L, i n)$ ) are less than per-capita transfers type $l$ agents make under $C E L$ (i.e., $\left.S_{l}(C E L, i n)\right)$ in this region. This implies that inequality conditions required for both types of agents to play in are satisfied more easily for $T A L$ than $C E L$ in this region (observe that the $T A L$ path of awards is closer to the $P$ path of awards than the $C E L$ path of awards in this region). Also note that as $E \rightarrow \sum_{i \in N} \frac{c_{i}}{2}$, it becomes easier to satisfy the inequality conditions in $T A L$. Hence, if $\sum_{i \in N} \frac{c_{i}}{2} \leq E \leq n_{h} c_{h}+\left(n_{l}-n_{h}\right) \frac{c_{l}}{2}$, the result in Theorem 1 is essentially valid: only difference is that for the specific case, $E=\sum_{i \in N} \frac{c_{i}}{2}, T A L$ coincides with $P$ and therefore the result in Theorem 1 holds also for the Talmud rule.

Moreno-Ternero \& Villar (2006) generalize the Talmud rule by introducing the TAL-family of rules, which contains the Talmud, constrained equal awards and the constrained equal losses rules as special cases.

Definition 6 (TAL-family) The TAL-family consists of all rules of the following form: For some $\theta \in[0,1]$, for all $(E, C) \in \widetilde{\mathcal{B}}$ and for all $i \in N$,

$$
F_{i}^{\theta}(C, E)=\left\{\begin{array}{c}
\min \left\{\theta c_{i}, \lambda\right\} \text { if } E \leq \sum_{i \in N} \theta c_{i} \\
\max \left\{\theta c_{i}, c_{i}-\mu\right\} \text { if } E \geq \sum_{i \in N} \theta c_{i}
\end{array}\right.
$$

where $\mu$ and $\lambda$ solve $\sum_{i \in N} F_{i}^{\theta}(C, E)=E$.

Note that $F=C E L$ for $\theta=0, F=C E A$ for $\theta=1$ and $F=T$ for $\theta=1 / 2$. Moreover, for all $\theta \in[0,1], F^{\theta}$ coincides with the constrained equal awards rule (on adjusted $\theta$-claims) if $E \leq \sum_{i \in N} \theta c_{i}$ and the constrained equal losses rule (on adjusted $(1-\theta)$-claims) if $E \geq \sum_{i \in N} \theta c_{i}$ (see Moreno-Ternero \& Villar, 2006). On the other hand, note that $P$ is not a member of the TAL-family. If we change the game by including all members of the TAL-family along with the proportional rule in the strategy space of the company, the extended game $\widetilde{\Gamma}$ has infinitely many subgames which are $\Gamma^{P}$ and, for all $\theta \in[0,1], \Gamma^{\theta}$. We can argue, along the same lines as the proof of Proposition 2, that for any bankruptcy problem $(E, C) \in \widetilde{\mathcal{B}}$, there always exists a subgame perfect Nash equilibrium of $\widetilde{\Gamma}$ with the proportional rule and the member of the TAL-family parametrized by $\theta^{*}=\frac{1+r_{f}}{1+r}$. Given $r$ and $r_{f}, \frac{E}{\sum_{i \in N} c_{i}}=\frac{1+r_{f}}{1+r} \in\left[\frac{1}{2}, 1\right)$. There exists a $\theta^{*} \in\left[\frac{1}{2}, 1\right]$ such that $\theta^{*}=\frac{1+r_{f}}{1+r}$, for which the awards vector under rule $F^{\theta^{*}}$ coincides with the awards vector under the proportional rule. Any other member of the TAL-family coincides either with the constrained equal awards rule or the constrained equal losses rule (both on adjusted claims) given $r$ and $r_{f}$. Therefore, a result similar to the one in Proposition 2 would follow.

\subsection{Multiple Companies and Competition}

So far we assume a single company for simplicity. However, in many cases there are more than one company competing for the same group of investors in real life. In this subsection, we introduce

\footnotetext{
${ }^{13} T A L_{i}\left(\left(c_{1}, \ldots, c_{n}\right), E\right)=\frac{c_{i}}{2}+C E L_{i}\left(\left(\frac{c_{1}}{2}, \ldots, \frac{c_{n}}{2}\right), E-\sum_{i \in N} \frac{c_{i}}{2}\right)$
} 
the competition between two companies. Below, we first set up the model with two companies and then present our results.

In this subsection, we assume that there are two companies ( $\bar{m}$ and $\underline{m}$ ) which are competing for the same set of potential investors in $N_{l} \cup N_{h}$. We model the competition between these two companies à la Stackelberg. Accordingly, $\bar{m}$ is the first-mover and $\underline{m}$ is the second-mover. Risk-return characteristics $\left(\pi_{s}, r\right.$, and $\left.r_{f}\right)$ are assumed to be identical for both companies and there is, again, a single risk-free asset (with return $\bar{r}$ ) available for agents. As in our base model, each company chooses a bankruptcy rule out of three $(P, C E A$, and $C E L)$ rules to maximize its investment volume. However, now $\bar{m}$ moves first and chooses a bankruptcy rule and then $\underline{m}$, observing $\bar{m}$ 's action, moves and also chooses a bankruptcy rule. Accordingly, $\bar{m}$ 's strategy space is denoted by $\psi_{\bar{m}}=\{P, C E A, C E L\}$ and $\underline{m}$ 's strategy space is denoted by $\psi_{m}=\{(P, P),(C E A, P),(C E L, P)$, $(P, C E A),(C E A, C E A),(C E L, C E A),(P, C E L),(C E A, C E L),(C E L, C E L)\}$ where first entries refer to $\bar{m}$ 's actions and second entries refer to $\underline{m}$ 's actions. Each action couple (consisting of actions of both companies) starts a subgame to be played by agents. We denote the subgame started by $\bar{m}$ playing $F_{\bar{m}}$ and $\underline{m}$ playing $F_{\underline{m}}$ by $\Gamma^{F_{\bar{m}}, F_{\underline{m}}}$. After observing companies' actions, agents simultaneously choose whether to invest in one of the companies or in the risk-free outside asset. ${ }^{14}$ Since there are two companies now, agents $i$ 's action set is $a_{i}=\left(\bar{m}, \underline{m}\right.$,out). For all $i \in N_{l} \cup N_{h}$, we denote the actions taken by agent $i$ in subgame $\Gamma^{F_{\bar{m}}, F_{\underline{m}}}$ as $a_{i, \Gamma_{\bar{m}} F_{\underline{m}}}$. We describe what each agent $i$ does in each subgame $\Gamma^{F_{\bar{m}}, F_{\underline{m}}}$ by agent $i$ 's strategy, which is again denoted by $s_{i} \in \psi_{i}$. Agent $i$ 's strategy space, $\psi_{i}$, can be written as

$$
\psi_{i}=\left\{\left(a_{i, \Gamma_{\bar{m}} F_{\underline{m}}}\right) \mid F_{\bar{m}}, F_{\underline{m}} \in\{P, C E A, C E L\} \text { and } a_{i, \Gamma_{\bar{m}}, F_{\underline{m}}} \in\{\bar{m}, \underline{m}, o u t\}\right\} .
$$

We denote $\bar{m}$ 's payoff function as $V_{\bar{m}}\left(F_{\bar{m}}, F_{\underline{m}}\right)=\sum_{t \in\{l, h\}} n_{t, \bar{m}}\left(F_{\bar{m}}, F_{\underline{m}}\right) w_{t}$, where $n_{t, \bar{m}}$ stands for the number of type $t$ agents who invest in $\bar{m}$ in the subgame $\Gamma^{F_{\bar{m}}, F_{\underline{m}}}$ and $\underline{m}$ 's payoff function as $V_{\underline{m}}\left(F_{\bar{m}}, F_{\underline{m}}\right)=\sum_{t \in\{l, h\}} n_{t, \underline{m}}\left(F_{\bar{m}}, F_{\underline{m}}\right) w_{t}$, where $n_{t, \underline{m}}$ stands for the number of type $t$ agents who invest in $\underline{m}$ in the subgame $\Gamma^{F_{\bar{m}}, F_{\underline{m}}}$. Therefore, we can write the objective function for company $m \in\{\underline{m}, \bar{m}\}$ as

$$
\max _{F_{m} \in\{P, C E A, C E L\}} V_{m}\left(F_{m}, .\right) .
$$

We, again, focus on pure strategy subgame perfect Nash equilibria of this game. Moreover, throughout this subsection, we focus on equilibria, which satisfy the following refinement assumptions:

Assumption 1: Each agent plays his weakly dominant strategy in case there is one. ${ }^{15}$

Assumption 2: When a certain type of agents are indifferent between two companies, half of them invest in $\bar{m}$ and the other half in $\underline{m} .{ }^{16}$

Table 5 defines the sequential game $\bar{\Gamma}$.

\footnotetext{
${ }^{14}$ Each agent can invest in only one investment alternative, as it was in the base model.

${ }^{15}$ This is a fairly common assumption in the political economy, elections, and voting literature.

${ }^{16}$ This is a frequently made assumption in the industrial organization literature (e.g., Hotelling's game). For this assumption, we, further, need to assume that there are even number of agents of each type.
} 


\begin{tabular}{||c|c||}
\hline \hline Players & $\{\bar{m}, \underline{m}\} \cup N_{l} \cup N_{h}$ \\
\hline Strategies & $\psi_{\bar{m}} \times \psi_{\underline{m}} \prod_{i \in N_{l} \cup N_{h}} \psi_{i}$ \\
\hline Payoffs & $\left(V_{\bar{m}}\left(F_{\bar{m}}, F_{\underline{m}}\right), V_{\underline{m}}\left(F_{\bar{m}}, F_{\underline{m}}\right)\left(V_{i, s_{i}}\left(F_{\bar{m}}, F_{\underline{m}}, s_{-i}\right)\right), i \in N_{l} \cup N_{h}\right.$ \\
\hline \hline
\end{tabular}

Table 5: Sequential Game $\bar{\Gamma}$.

where $s_{-i}$ denotes all agents' strategies except agent $i$.

Below, we list our main findings. Formal proofs for these results can be found in Appendix C where we characterize all Nash equilibria in each subgame and all subgame perfect Nash equilbria of the extended model. In the following, we denote the total amount of money held by type $l$ agents (i.e., $\left.\sum_{i \in N_{l}} w_{i}\right)$ as $W_{l}$ and the total amount of money held by type $h$ agents (i.e., $\sum_{i \in N_{h}} w_{i}$ ) as $W_{h}$. (i) The income distribution is very influential on companies' decisions and hence the bankruptcy rules chosen in equilibrium. In all subgame perfect Nash equilibria (except the ones that involve all two-combinations of three rules), $\bar{m}$, i.e., the first-mover, chooses a bankruptcy rule favoring type of agents with a larger $W_{t}$.

(ii) In all subgame perfect Nash equilibria (except the ones that involve all two-combinations of three rules), $\underline{m}$, i.e., the second-mover, follows the first-mover by choosing the same bankruptcy rule when $W_{h} \geq 2 W_{l}$ (or $W_{l} \geq 2 W_{l}$ ). On the other hand, when $2 W_{l} \geq W_{h} \geq W_{l}$ (or $2 W_{h} \geq W_{l} \geq W_{h}$ ), the first-mover and the second-mover choose different bankruptcy rules in which case the first-mover has an advantage in terms of the amount of investments attracted.

(iii) Under some circumstances (e.g., $W_{h} \geq 2 W_{l}$ or $W_{l} \geq 2 W_{l}$ ), the competition between two companies leads to a smaller amount of total investment compared to the single-company case. In such circumstances, $\underline{m}$ follows $\bar{m}$ by choosing the same rule that favors a certain type of agents. The other type of agents do not invest in a company leading to a lower total investment compared to the investment volume in the one-company case.

(iv) The type of agents with a larger $W_{t}$ cannot obtain a payoff advantage over the other type of agents, even though the first-mover chooses a bankruptcy rule that favors them in all generic cases. When $2 W_{l} \geq W_{h} \geq W_{l}$ (or $2 W_{h} \geq W_{l} \geq W_{h}$ ), companies choose different bankruptcy rules and each type of agent invests in the company whose bankruptcy rule favors his type (if they invest in a company at all). Hence, there are no transfers between two types of agents. When $W_{h} \geq 2 W_{l}$ (or $W_{l} \geq 2 W_{l}$ ), both companies choose the same bankruptcy rule (the one that favors the type of agents with a larger $W_{t}$ ) and only the type of agents who are favored by this rule invest in companies. Hence, again, there are no transfers between two types of agents.

Our results in this subsection show that incorporating the competition for funds between borrowers (companies) has a critical influence on bankruptcy rules chosen in equilibrium. Although, the equilibria we find in this extended model do not provide a noncooperative support for the proportional rule, we show that the use of the proportional rule in the one-company case can attract more investment compared to the two-company case, which involves other rules (see iii above). Similarly, if a central authority implements the proportional rule in the two-company case, this can attract more investment to these two companies than a liberal regime that allows companies to choose their bankruptcy rules strategically (as modeled above). This can be considered as a rationale for a policymaker who aims to maximize the investment volume in the economy, to implement the proportional rule in the two-company case. 


\section{Comparative Static Analysis}

In this section, we conduct comparative static analyses on the risk-return and income distribution parameters. We show how the equilibrium investment in the company responds to changes in these parameters. First of all, we present the closed form versions of the inequalities that determine the equilibrium under each rule. This will help us in conducting comparative static analyses.

Recall that we have only $(i n, i n)$ and (out, out) as (representative) subgame perfect Nash equilibrium action profiles for agents. Therefore, in the following we provide the closed form expressions of the inequalities that lead to these subgame perfect equilibria.

\section{The Proportional Rule:}

For $(i n, i n)$ equilibrium,

$$
P_{t}^{e} \geq V_{t, \text { out }}
$$

is required. It can be written explicitly as

$$
\begin{aligned}
\pi_{s}(1+r) w_{t}+\left(1-\pi_{s}\right)\left(1+r_{f}\right) w_{t} & \geq(1+\bar{r}) w_{t} \\
\pi_{s} r+r_{f}-\pi_{s} r_{f} & \geq \bar{r} \\
\pi_{s}\left(r-r_{f}\right) & \geq \bar{r}-r_{f} \\
\pi_{s} & \geq \frac{\bar{r}-r_{f}}{r-r_{f}} .
\end{aligned}
$$

Obviously, $\pi_{s}<\frac{\bar{r}-r_{f}}{r-r_{f}}$ leads to (out, out) equilibrium. Moreover, recall that $\pi_{s}<\frac{\bar{r}-r_{f}}{r-r_{f}}$ also leads to (out, out) equilibrium under $C E A$ and $C E L$ since $\pi_{s} \geq \frac{\bar{r}-r_{f}}{r-r_{f}}$ is a necessary condition for any agent to play in under any rule.

The Constrained Equal Awards Rule:

For $(i n, i n)$ equilibrium

$$
\begin{gathered}
P_{t}^{e} \geq V_{t, \text { out }} \text { and } \\
P_{h}^{e}+\left(1-\pi_{s}\right) S_{h}(C E A, \text { in }) \geq V_{h, \text { out }}
\end{gathered}
$$

is required. We consider two cases to express the second inequality explicitly under $C E A$ :

(a-1) If $c_{h}>c_{l}>\lambda_{c e a}$, then $P_{h}^{e}+\left(1-\pi_{s}\right) S_{h}(C E A, i n) \geq V_{h, o u t}$ can be written as,

$$
\pi_{s}(1+r) w_{h}+\left(1-\pi_{s}\right)\left(1+r_{f}\right) w_{h}+\left(1-\pi_{s}\right) \frac{n_{l}}{n_{h}+n_{h}}\left[\left(1+r_{f}\right)\left(w_{l}-w_{h}\right)\right] \geq(1+\bar{r}) w_{h},
$$

which is equal to

$$
\frac{n_{l}}{n_{l}+n_{h}} \leq \frac{w_{h}}{w_{h}-w_{l}} \frac{\pi_{s} r+\left(1-\pi_{s}\right) r_{f}-\bar{r}}{\left(1-\pi_{s}\right)\left(1+r_{f}\right)}
$$

(a-2) If $c_{h}>\lambda_{\text {cea }} \geq c_{l}$, then $P_{h}^{e}+\left(1-\pi_{s}\right) S_{h}(C E A$, in $) \geq V_{h, \text { out }}$ can be written as,

$$
\pi_{s}(1+r) w_{h}+\left(1-\pi_{s}\right)\left(1+r_{f}\right) w_{h}+\left(1-\pi_{s}\right) \frac{n_{l}}{n_{h}}\left(r_{f}-r\right) w_{l} \geq(1+\bar{r}) w_{h},
$$


which is equal to

$$
\frac{n_{l}}{n_{h}} \leq \frac{w_{h}}{w_{l}} \frac{\pi_{s} r+\left(1-\pi_{s}\right) r_{f}-\bar{r}}{\left(1-\pi_{s}\right)\left(r-r_{f}\right)} .
$$

The Constrained Equal Losses Rule:

For $(i n, i n)$ equilibrium

$$
\begin{gathered}
P_{t}^{e} \geq V_{t, \text { out }} \text { and } \\
P_{l}^{e}+\left(1-\pi_{s}\right) S_{l}(C E L, \text { in }) \geq V_{l, \text { out }}
\end{gathered}
$$

is required. We consider two cases to express the second inequality explicitly under $C E L$ :

(b-1) If $c_{h}>c_{l}>\lambda_{c e l}$, then $P_{l}^{e}+\left(1-\pi_{s}\right) S_{l}(C E L$, in $) \geq V_{l, o u t}$ can be written as,

$$
\pi_{s}(1+r) w_{l}+\left(1-\pi_{s}\right)\left(1+r_{f}\right) w_{l}+\left(1-\pi_{s}\right) \frac{n_{h}}{n_{h}+n_{l}}\left[\left(r-r_{f}\right)\left(w_{l}-w_{h}\right)\right] \geq(1+\bar{r}) w_{l},
$$

which is equal to

$$
\frac{n_{h}}{n_{h}+n_{l}} \leq \frac{w_{l}}{w_{h}-w_{l}} \frac{\pi_{s} r+\left(1-\pi_{s}\right) r_{f}-\bar{r}}{\left(1-\pi_{s}\right)\left(r-r_{f}\right)}
$$

(b-2) If $c_{h}>\lambda_{c e l} \geq c_{l}$, then $P_{l}^{e}+\left(1-\pi_{s}\right) S_{l}(C E L$, in $) \geq V_{l, \text { out }}$ can be written as,

$$
\pi_{s}(1+r) w_{l} \geq(1+\bar{r}) w_{l}
$$

which is equal to

$$
\pi_{s} \geq \frac{1+\bar{r}}{1+r}
$$

Note that $\pi_{s} \geq \frac{\bar{r}-r_{f}}{r-r_{f}}$ is already implicitly embedded in (10), (11), (12) and (13) also implies it. Hence practically, we do not have two conditions determining the $(i n, i n)$ equilibrium under $C E A$ and $C E L$. Below, we present a table consisting of parameters $r, r_{f}, \bar{r}, \pi_{s}, n_{l}, n_{h}, w_{l}$, and $w_{h}$ and the effects of changes in those parameters on inequalities that determine the subgame perfect equilibrium investment in the company. In particular, + in a cell means that the inequality in the corresponding column will still be valid after an increase in the respective parameter and - in a cell means that the inequality in the corresponding column might not be valid after an increase.

\begin{tabular}{c|ccccc} 
variable/inequality & $(9)$ & $(10)$ & $(11)$ & $(12)$ & $(13)$ \\
\hline$r$ & + & + & + & + & + \\
$r_{f}$ & + & + & + & + & no change \\
$\bar{r}$ & - & - & - & - & - \\
$\pi_{s}$ & + & + & + & + & + \\
$n_{l}$ & no change & - & - & + & no change \\
$n_{h}$ & no change & + & + & - & no change \\
$w_{l}$ & no change & + & - & + & no change \\
$w_{h}$ & no change & - & + & - & no change
\end{tabular}

Table 6: Comparative Static Analysis on the Set of SPNE 
The following proposition shows the relationship between risk-return parameters and the equilibrium investment in the company.

Proposition 3 A change in $r, r_{f}, \bar{r}$ and $\pi$ can lead to only two types of changes in the equilibrium investment:

(i) Either the equilibrium investment in the company decreases from $\sum_{t \in\{l, h\}} n_{t} w_{t}$ to 0 , or

(ii) the equilibrium investment in the company increases from 0 to $\sum_{t \in\{l, h\}} n_{t} w_{t}$.

Proof. In all subgame perfect Nash equilibria, there are only two possible levels of investment: 0 and $\sum_{t \in\{l, h\}} n_{t} w_{t}$. Hence, if a change in $r, r_{f}, \bar{r}$ and $\pi$ causes a change in the equilibrium investment it can be either a movement from $\sum_{t \in\{l, h\}} n_{t} w_{t}$ to 0 , or a movement from 0 to $\sum_{t \in\{l, h\}} n_{t} w_{t}$.

Proposition 4 An increase in $r, r_{f}$ or $\pi_{s}$ can never decrease the equilibrium investment in the company.

Proof. The proof is simple and hence omitted. It follows from the fact that agents' expected payoff from playing in is increasing in $r, r_{f}$ and $\pi_{s}$.

There is a large literature pointing out advantages of equity financing (shareholdership) over bank loans. Some governments (e.g., Turkey) use taxes on different investment alternatives with the aim of encouraging equity financing and shareholdership (e.g., reducing taxes on stock market earnings). Our result above points towards this direction. It shows that taxes on earnings from the investment in the company can be used as a policy tool by the government to increase the shareholdership in the economy. In particular, the government can increase the number of shareholders as well as the investment in the company by decreasing the taxes on shareholder earnings. On the other hand, the return from the risk-free outside asset $(\bar{r})$ enters all inequalities above from the right side with a positive sign. Therefore, an increase in $\bar{r}$ increases the attractiveness of the risk-free asset i.e. playing out. Accordingly, the equilibrium investment in the company weakly decreases as a result of an increase in $\bar{r}$. Similar to the policy implication about $r$ and $r_{f}$ above, taxes on $\bar{r}$ can also be used as a policy tool. The government can increase the investment in the company by increasing the taxes on the risk-free asset (e.g., increasing taxes on interest earnings).

The following proposition shows the relationship between income distribution parameters and the equilibrium investment in the company.

Proposition 5 Decreases in $n_{l}, n_{h}, w_{l}$ and $w_{h}$ can never increase the equilibrium investment in the company.

Proof. The proof is simple and follows from the following facts:

(i) The proportional rule is always a part of the equilibrium.

(ii) The condition determining the equilibrium strategies under the proportional rule is independent of the income distribution parameters (i.e., $n_{l}, n_{h}, w_{l}$ and $w_{h}$ ) as shown in Lemma 6 .

(iii) If $P_{t}^{e}<V_{t, \text { out }}$, the equilibrium investment in the company is 0 and does not change due to any change in $n_{l}, n_{h}, w_{l}$ and $w_{h}$.

(iv) If $P_{t}^{e} \geq V_{t, \text { out }}$, the equilibrium investment in the company under $P$ is $\sum_{t \in\{l, h\}} n_{t} w_{t}$. Since the change in $\sum_{t \in\{l, h\}} n_{t} w_{t}$ with respect to a change in $n_{l}, n_{h}, w_{l}$ or $w_{h}$, a decrease in any of these parameters decreases the equilibrium investment in the company. 
Needless to say, increases in $n_{l}, n_{h}, w_{l}$ and $w_{h}$ can never decrease the equilibrium investment in the company.

The following corollary shows the relationship between the impact a change in parameters has on the equilibrium investment in the company under $P$ and the impact it has on the subgame perfect equilibrium level of investment in the company.

Corollary 6 The equilibrium investment volume changes only as a result of a change in parameters that affect the investment volume under the proportional rule.

Proof. The result follows from the fact that the proportional rule is the only rule that is always a part of the subgame perfect Nash equilibrium. Hence, if the equilibrium investment in the company under $P$ does not change as a result of a change in parameters, the subgame perfect equilibrium level of investment in the company does not change.

This corollary implies that if the equilibrium investment in the company under $C E A$ or $C E L$ drops as a result of a change in some parameter, this does not necessarily mean that the subgame perfect equilibrium level of investment in the company drops, since $P$ might still be leading to $n_{l} w_{l}+n_{h} w_{h}$ investment in the company. For example, as a result of a change in some parameter the investment in the company under $C E A(C E L)$ might drop from $n_{l} w_{l}+n_{h} w_{h}$ to $n_{l} w_{l}\left(n_{h} w_{h}\right)$, but under $P$ it might be still $n_{l} w_{l}+n_{h} w_{h}$. In that case, the subgame perfect equilibrium investment in the company is still $n_{l} w_{l}+n_{h} w_{h}$. The equilibrium investment in the company drops to 0 only if $\pi_{s} \geq \frac{\bar{r}-r_{f}}{r-r_{f}}$ changes to $\pi_{s}<\frac{\bar{r}-r_{f}}{r-r_{f}}$ as a result of a change in some parameter. Hence, there are still two possible equilibrium investment levels: $n_{l} w_{l}+n_{h} w_{h}$ and 0 . Similarly, the equilibrium investment may not rise as a result of a change in some parameter, even if it causes an increase in the investment under $C E A$ or $C E L$.

\section{Discussion}

In this section, we discuss our assumptions, the way we set up our model, our results, extension ideas and possible future research. For instance, we assume identical risk-attitudes (i.e., risk neutrality) for all agents. Our main purpose in this research is to integrate the models of bankruptcy problem into a noncooperative game theoretical model that involves strategic decision-making under uncertainty. Incorporating the simple bankruptcy model into a strategic context requires some simplifications such as the ones we mentioned above. Below, we discuss some of our assumptions and possible extensions.

Individual Optimization on the Level of Investment: In this paper, we assume that individuals do not maximize their expected payoffs by choosing the amount of money they want to invest in the company and in the savings account. They either invest in the company or deposit their money in a savings account. However, if we allowed them to optimize under the risk-neutrality assumption, we would have corner solutions where each agent, again, will either fully invest in the company or in the savings account. Hence, our assumption on investment decision, is not very restrictive under the risk-neutrality assumption.

$T$ number of types: A natural question that arises is whether our results in the base model are valid in a model with any finite number of types. If we assume $T$ types and one agent per each type, by 
using Lemmas 1, 3, 6 and, 8 we can obtain results identical to the ones in the base model. On the other hand, our ultimate goal is to extend our model to a more general setting, which includes $T$ types and $n_{t}$ number of type $t$ agents. This is far from being trivial and is work in progress. The main difficulty here is that when the number of certain type of agents change (used in our proofs), the identity (e.g., transfer-maker or transfer-receiver) of some other types may also change, which was not the case in the setting with two types. Hence, proofs should take these potential changes into account. Nevertheless, we conjecture that our results are still valid in this extended model, mainly due to the order-preserving nature of the bankruptcy rules used.

Distributive Properties: In a follow-up paper (see Karagözoğlu, 2008), we analyze some distributive properties such as minimal rights first, securement of initial investments, initial investments first, reasonable lower bounds on awards, and reasonable lower bounds on losses in the class of bankruptcy problems with endogenous estates. We show that the proportional rule receives more support compared to the constrained equal awards and the constrained equal losses rules. Some normative properties that were not satisfied by the proportional rule in the general class of bankruptcy problems are satisfied by this rule in the class of bankruptcy problems with an endogenous estate. These changes are mostly due to the fact that estate and claims are not completely independent in this class. As a result, certain values of the estate for which the proportional rule does not satisfy certain properties are eliminated in this class of bankruptcy problems.

Bankruptcy Laws and Sequential Priority Rules: We observe that many bankruptcy laws apply certain sequential priority rules in the liquidation of bankrupt companies' assets. Priority classes are defined on the basis of seniority (e.g., founding stockholders or new stockholders), status of creditors (e.g., stockholder or bondholder) or status of claims (e.g., secured or unsecured) etc. The American bankruptcy law is a frequently quoted example in which priority classes are federal government (taxes), trustees (administrative expenses of the trustee) and two kinds of creditors (secured and unsecured claims). These rules and the constrained equal awards and constrained equal losses rules have a commonality: in all of these rules, loosely speaking, there are people favored by the rule (i.e., receiving transfers) and there are people disfavored by the rule (i.e., making transfers). In this paper, we show that the strength of the proportional rule stems from its "neutrality" (zero transfers as a benchmark). Therefore, if we slightly modify our model to capture priority criteria such as seniority or status, there will, again, exist some parameter values for which, some agents do not invest in the company in equilibrium under the sequential priority rules. Loosely speaking, the proportional rule will outperform sequential priority rules in attracting investment to the company. Moreover, an important fact, which is in conformity with our results is that the proportional rule is applied in the liquidation process within each priority class in sequential priority rules.

Increasing Returns and/or Decreasing Risk: In certain real-life circumstances, one might suggest that an increase in the investment volume can lead to an increase in the return rate (e.g., the investment project involves increasing returns to capital) and/or a decrease in the rate of risk (e.g., a higher level of capital increases the likelihood of success). If we incorporate these two possibilities, we expect that our results would quantitatively change. The relative (with respect to the proportional rule) positions of the constrained equal awards and the constrained equal losses rules would improve. However, we expect no qualitative change in the results, i.e., the proportional 
rule would still have an advantage over others. ${ }^{17}$

Nonexistence of Pure Strategy Nash Equilibrium Under Different Risk Attitudes: If we assume that the high type agents are risk-neutral and the low type agents are risk-averse, we show that a pure strategy Nash Equilibrium might not exist under some parameter values. Below, we explain the dynamics of the non-existence result in more detail.

The reason of non-existence under different risk attitudes (in particular, risk neutral type $h$ agents and risk averse type $l$ agents) is the high degree of conflict of interest and the availability of an escape option (i.e., the risk-free asset) causing cyclicity. When we analyze the parameter conditions under which there is no pure strategy equilibrium, we see that the cost that is imposed on type $h$ agents (type $l$ agents) under $C E A(C E L)$ can be understood as the level of conflict of interest. For example, under $C E A$, if $c_{h}>\lambda_{\text {cea }}>c_{l}$, then the term $\frac{n_{l}}{n_{h}}\left(r_{f}-r\right) w_{l}$ shows per-type $h$-capita transfer to type $l$ agents. A quick look at the term shows that if $n_{h}$ is high, then per-type $h$-capita transfer is low, if $n_{l}$ is high it is high and if $\left(r_{f}-r\right)$ is high, it is again high. Therefore, for some parameter values, under $C E A$, type $h$ agents do not play in against type $l$ agents playing in. Hence, their best response to type $l$ agents playing in is to play out. And type $l$ agents' best response to type $h$ agents playing out is also out. However, type $h$ agents' best response to type $l$ agents playing out is to play in and type $l$ agents' best response to type $h$ agents playing in is to play in. Hence, the process does not stop at an equilibrium. In the following, we construct an example in which there is no pure strategy Nash equilibrium under $C E A$.

Example 2 Assume that $n_{l}=n_{h}=1, w_{l}=10, w_{h}=100, r=0.8, r_{f}=0.2$, and $\bar{r}=0.61$. Moreover, recall that type $h$ agent is risk neutral whereas type $l$ agent is risk-averse. This implies that type $l$ agent rejects fair gambles. Then, we can simply calculate the expected payoffs for the type $h$ agent as

$$
\begin{aligned}
P_{h}^{e} & =(0.7)(1+0.8) 100+(0.3)(1+0.2) 100=162 \text { and } \\
C E A_{h}^{e} & =(0.7)(1+0.8) 100+(0.3) 114=160.2 .
\end{aligned}
$$

Denote the utility function of the type $l$ agent as $U($.$) . Since$

$$
\begin{aligned}
V_{h, \text { out }} & =161, \\
V_{l, \text { out }} & =16.1, \text { and } \\
C E A_{l} & =18
\end{aligned}
$$

the payoff matrix can be written as

\begin{tabular}{|c|c|c|}
\hline$h \backslash l$ & in & out \\
\hline in & $160.2, U(18)$ & $162, U(16.1)$ \\
\hline out & $161,(0.7) U(18)+(0.3) U(12)$ & $161, U(16.1)$ \\
\hline
\end{tabular}

Risk-aversion implies that $(0.7) U(18)+(0.3) U(12)<U(16.2)$. If $(0.7) U(18)+(0.3) U(12)<$ $U(16.1)$ is also valid, then we do not have pure strategy Nash equilibrium in this game. $B R_{h}(i n)=$

\footnotetext{
${ }^{17}$ A similar increasing returns idea was analyzed by Izquierdo \& Rafels (1996) who characterized the proportional division in financial (or deposit) games.
} 
out, $B R_{l}($ out $)=$ out, $B R_{h}($ out $)=$ in and $B R_{l}($ in $)=i n$. Hence, there is a cycle. As the reader might realize, the statement " $P_{h}^{e} \geq V_{h, \text { out }}$ if and only if $U\left(P_{l}\right) \geq V_{l, \text { out }}$ " is not valid anymore, which causes the cycle. The nonexistence result Kibris \& Kibris (2008) have under $C E A$ in a similar setup (with potentially different risk attitudes) is also in line with our arguments here.

Nonexistence of Pure Strategy Nash Equilibrium Under Different Outside Asset Payoffs: We assumed that the risk-free asset brings the same payoff to both types of agents in case they invest in it. This is an unbiased (neutral) assumption to make. However there might be real life instances in which there are different risk-free assets available to different types of agents (e.g., the risk-free asset available to $h$ agents might pay higher (lower) than the risk-free asset available to type $l$ agents). In that case, the company's decision in equilibrium might be different than the one we described above. However, a problem with this more general approach is that, again, it can bring non-existence results for some parameter values. Hence, we kept our neutral assumption that riskfree asset pays the same return rate to all agents. In the following, we construct an example where there is no pure strategy Nash equilibrium in the subgame under $C E A$.

Example 3 Assume that $n_{l}=n_{h}=1, w_{l}=10, w_{h}=100, r=0.8, r_{f}=0.2, \bar{r}_{h}=0.61$ and $\bar{r}_{l}=0.63$. Then, we can simply calculate the expected payoffs as

$$
\begin{aligned}
P_{h}^{e} & =(0.7)(1+0.8) 100+(0.3)(1+0.2) 100=162, \\
C E A_{h}^{e} & =(0.7)(1+0.8) 100+(0.3) 114=160.2, \\
P_{l}^{e} & =(0.7)(1+0.8) 10+(0.3)(1+0.2) 10=16.2, \text { and } \\
C E A_{l}^{e} & =(0.7)(1+0.8) 10+(0.3) 18=18 .
\end{aligned}
$$

Risk-free asset brings payoffs $V_{h, \text { out }}=161$ and $V_{l, \text { out }}=16.3$. Hence, the payoff matrix can be written as

\begin{tabular}{|c|c|c|}
\hline$h \backslash l$ & in & out \\
\hline in & $160.2,18$ & $162,16.3$ \\
\hline out & $161,16.2$ & $161,16.3$ \\
\hline
\end{tabular}

As the reader can notice, type $h$ agent does not play in against type $l$ agents playing $i n$. Hence, his best response to type $l$ agent playing in is to play out. And type $l$ agent's best response to type $h$ agent playing out is also out. However, type $h$ agent's best response to type $l$ agent playing out is to play in and type $l$ agent's best response to type $h$ agent playing in is to play in. Hence, the process does not stop at an equilibrium. As the reader might realize, it is again the invalidity of the statement " $P_{h}^{e} \geq V_{h, \text { out }}$ if and only if $P_{l}^{e} \geq V_{l, \text { out }}$ ", which causes the cycle.

\section{Conclusion}

In this paper, we introduced a new class of bankruptcy problems, which have an empirical appeal. In these bankruptcy problems, the value of the estate to be allocated to agents is endogenous and depends on agents' investment decisions. This is what we observe in many real life cases. For instance the amount to be allocated by a firm to its shareholders/stockholders may depend on the initial amount of money borrowed from them. Moreover, in line with some recent suggestions in 
favor of a more liberal bankruptcy law, which provides a menu of bankruptcy procedures and allows companies to select among them (see Hart, 2000), we allow the company in our model to choose from a menu of bankruptcy rules that consists of three well-known rules, i.e., the proportional rule, the constrained equal awards rule and the constrained equal losses rule. Company's objective in choosing a bankruptcy rule is to maximize the investment volume, which would lead to maximum profit. Agents observe the choice made by the company and decide whether to lend money to the company (risky investment) or deposit their money in a savings account (risk-free investment).

Our results show that the proportional rule receives a strong and robust support in this setting. There always exists a subgame perfect Nash equilibrium, which involves the proportional rule. A direct implication is that there is no equilibrium in which the proportional rule leads to a lower level of investment volume than the other rules. This result is independent of the income distribution in the economy and holds even under one-sided uncertainty on income distribution (i.e., the company does not know the income distribution perfectly). In fact, for probability distributions that assign non-zero probability to all possible income distributions, the proportional rule would be the unique optimal strategy for an expected payoff maximizing company. In a more complete setting where the company maximizes its profit by choosing a bankruptcy rule and a return rate, there exists a unique subgame perfect Nash equilibrium, which involves the proportional rule. We also extend the menu of rules to include the Talmud rule and show that results essentially do not change. Moreover, we argue, along the same lines, that our results are still valid in a game that contains TAL-family of rules that contains infinitely many rules some of which are the Talmud rule, the constrained equal awards rule and the constrained equal losses rule. We also extend our model to incorporate the competition between two companies over potential investors. This extension offers interesting results and insights. Equilibria in this extended model depend crucially on the income distribution. In particular, the type of agents with a higher total amount of money and the level of income inequality determine the bankruptcy rules chosen by companies. Results of this extension also show that the competition between two companies can lead to a lower investment volume in the economy compared to the one-company model, which involves the proportional rule. This also implies that a policymaker who aims to maximize the investment volume in the economy can use the proportional rule, instead of allowing the companies to choose their own rules strategically to maximize their own investment volumes.

Our results provide, at least, partial explanations from a strategic and policymaking points of view for the fact that the proportional rule is frequently used in the liquidation process of a bankrupt company's assets (e.g., Hart, 2000 and Kaminski, 2006). To the best of our knowledge, this is the first paper which models the bankruptcy rule determination as a sequential game between lenders (agents) and borrowers (companies). It is also the first paper, which embeds the classical bankruptcy problem in a decision-making under uncertainty environment. 


\section{References}

[1] Araujo, A. P. \& Páscoa, M. R. (2002) "Bankruptcy in a model of unsecured claims", Economic Theory, 20(3), pp. 455 - 481.

[2] Ashlagi, I., Karagözoğlu, E. \& Klaus, B. (2008) "Noncooperative support for the equal division in estate division problems", Harvard Business School NOM Working Papers \# 09-069.

[3] Atlamaz, M., Berden, C., Peters, H. \& Vermeulen, D. (2008) "Non-cooperative solutions to claims problems", Maastricht University, METEOR Research Memoranda \# 038.

[4] Aumann, R. \& Maschler, M. (1985) "Game theoretic analysis of a bankruptcy problem from the Talmud", Journal of Economic Theory, 36(2), pp. 195 - 213.

[5] Bergantiños, G. \& Sanchez, E. (2002) "The proportional rule for problems with constraints and claims", Mathematical Social Sciences, 43(2), pp. 225 - 249.

[6] Chambers, C. P. \& Thomson, W. (2002) "Group order preservation and the proportional rule for the adjudication of conflicting claims", Mathematical Social Sciences, 44(3), pp. 235 252.

[7] Chang, C. \& Hu, C-C. (2008) "A non-cooperative interpretation of the $f$-just rules of bankruptcy problems", Games and Economic Behavior, 63(1), pp. 133 - 144.

[8] Ching, S. \& Kakkar, V. (2001) "A market approach to the bankruptcy problem", mimeo, City University of Hong Kong.

[9] Chun, Y. (1988) "The proportional solution for rights problem", Mathematical Social Sciences, 15(3), pp. 231 - 246.

[10] Chun, Y. (1989) "A noncooperative justification for egalitarian surplus sharing", Mathematical Social Sciences, 17(3), pp. 245 - 261.

[11] Chun, Y. \& Lee, J. (2007) "On the convergence of the random arrival rule in large claims problems", International Journal of Game Theory, 36(2), pp. 259 - 273.

[12] Curiel, I., Maschler, M. \& Tijs, S. H. (1987) "Bankruptcy games", Zeitschrift fur Operations Research, 31(5), pp. A143 - A159.

[13] Dagan, N. \& Volij, O. (1993) "The bankruptcy problem: a cooperative bargaining approach", Mathematical Social Sciences, 26(3), pp. 287 - 297.

[14] Dagan, N., Serrano, R. \& Volij, O. (1997) "A noncooperative view of consistent bankruptcy rules", Games and Economic Behavior, 18(1), pp. 55 - 72.

[15] De Frutos, M. A. (1999) "Coalitional manipulations in a bankruptcy problem", Review of Economic Design, 4(3), pp. 255 - 272.

[16] Gächter, S. \& Riedl, A. (2005) "Moral property rights in bargaining with infeasible claims", Management Science, 51(2), pp. 249 - 263. 
[17] Gächter, S. \& Riedl, A. (2006) "Dividing justly in bargaining problems with claims", Social Choice and Welfare, 27(3), pp. 571 - 594.

[18] García-Jurado, I., González-Díaz, J. \& Villar, A. (2006) "A noncooperative approach to bankruptcy problems", Spanish Economic Review, 8(3), pp. 189 - 197.

[19] Hart, O. (2000) "Different approaches to bankruptcy", NBER Working Paper \# 7921.

[20] Herrero, C. (2003) "Equal awards vs. equal losses: duality in bankruptcy", In: Sertel M.R., Koray S. (eds.) Advances in Economic Design, Springer, Berlin Heidelberg New York.

[21] Herrero, C., Moreno-Ternero, J. D. \& Ponti, G. (2010) "On the adjudication of conflicting claims: an experimental study", Social Choice and Welfare, 34(1), pp. 145 - 179.

[22] Herrero, C. \& Villar, A. (2001) "The three musketeers: four classical solutions to bankruptcy problems", Mathematical Social Sciences, 42(3), pp. 307 - 328.

[23] Hougaard, J. L. \& Østerdal, L. P. (2005) "Inequality preserving rationing", Economics Letters, 87(3), pp. 355 - 360.

[24] Izquierdo, J. M. \& Rafels, C. (1996) "A generalization of the bankruptcy game: Financial cooperative games", Universidad de Barcelona, working paper \# E96/09.

[25] Ju, B-G. (2003) "Manipulation via merging and splitting in claims problems", Review of Economic Design, 8(2), pp. 205 - 215.

[26] Ju, B-G. \& Karagözoğlu, E. (2009) "Claims market and bankruptcy rules", unpublished manuscript.

[27] Ju, B-G., Miyagawa, E. \& Sakai, T. (2007) "Non-manipulable division rules in claims problems and generalizations", Journal of Economic Theory, 132(1), pp. 1 - 26.

[28] Kaminski, M. (2006) "Parametric rationing methods", Games and Economic Behavior, 54(1), pp. $115-133$.

[29] Karagözoğlu, E. (2008) "Distributive concerns in the bankruptcy problem with an endogenous estate", Maastricht University, METEOR Research Memoranda \# 032.

[30] Kıbris, Ö. \& Kıbris, A. (2008) "On the investment implications of bankruptcy laws", mimeo, Sabanc1 University.

[31] Moreno-Ternero, J. D. (2002) "Noncooperative support for the proportional rule in bankruptcy problems", mimeo, Universidad de Alicante.

[32] Moreno-Ternero, J. D. (2006) "Proportionality and non-manipulability in bankruptcy problems", International Game Theory Review, 8(1), pp. 127 - 139.

[33] Moreno-Ternero, J. D. (2009) "The proportional rule for multi-issue bankruptcy problems", Economics Bulletin, 29(1), pp. 483 - 490..

[34] Moreno-Ternero, J. D. \& Villar, A. (2006) "The TAL-family of rules for bankruptcy problems", Social Choice and Welfare, 27(2), pp. 231 - 249. 
[35] Moulin, H. (2000) "Priority rules and other asymmetric rationing methods," Econometrica, $68(3)$, pp. $643-684$.

[36] Moulin, H. (2002) "Axiomatic cost and surplus sharing" in: Arrow K., Sen A., Suzumura K. (eds.) Handbook of Social Choice and Welfare, vol. 1, Elsevier, Amsterdam.

[37] O`Neill, B. (1982) "A problem of rights arbitration from the Talmud", Mathematical Social Sciences, 2(4), pp. 345 - 371.

[38] Thomson, W. (2003) "Axiomatic and game-theoretic analysis of bankruptcy and taxation problems: a survey", Mathematical Social Sciences, 45(3), pp. 249 - 297.

[39] Thomson, W. (2006) How to Divide When There isn't Enough; From the Talmud to Game Theory, unpublished manuscript.

[40] Young, P. (1985) "Cost Allocation" in: Young P. (eds.) Fair Allocation. Proceedings of Symposia in Applied Mathematics, vol. 33, Providence, RI: The American Mathematical Society.

[41] Yaari, M. E. \& Bar-Hillel, M. (1984) "On dividing justly", Social Choice and Welfare, $1(1)$, pp. $1-24$. 


\section{Appendix:}

\section{A - Comparative Static Analysis Calculations}

\section{Closed Form Expressions for Transfers}

$$
\begin{aligned}
S_{h}(C E A, \text { in }) & =\frac{n_{l}\left(r_{f}-r\right) w_{l}}{n_{h}} \text { if } c_{h}>\lambda_{\text {cea }} \geq c_{l} \\
S_{h}(C E A, \text { in }) & =\frac{n_{l}}{n_{h}+n_{l}}\left(1+r_{f}\right)\left(w_{l}-w_{h}\right) \text { if } c_{h}>c_{l} \geq \lambda_{c e a} \\
S_{l}(C E A, \text { in }) & =\left(r-r_{f}\right) w_{l} \text { if } c_{h}>\lambda_{c e a} \geq c_{l} \\
S_{l}(C E A, i n) & =\frac{n_{l}}{n_{h}+n_{l}}\left(1+r_{f}\right)\left(w_{h}-w_{l}\right) \text { if } c_{h}>c_{l} \geq \lambda_{c e a} \\
S_{l}(C E L, i n) & =\frac{n_{h}}{n_{h}+n_{l}}\left(r-r_{f}\right)\left(w_{l}-w_{h}\right) \text { if } c_{h}>c_{l}>\lambda_{c e l} \\
S_{l}(C E L, i n) & =-\left(1+r_{f}\right) w_{l} \text { if } c_{h}>\lambda_{c e l} \geq c_{l} \\
S_{h}(C E L, i n) & =\frac{n_{l}}{n_{h}+n_{l}}\left(r-r_{f}\right)\left(w_{h}-w_{l}\right) \text { if } c_{h}>c_{l}>\lambda_{c e l} \\
S_{h}(C E L, i n) & =\frac{n_{l}}{n_{h}}\left(1+r_{f}\right) w_{l} \text { if } c_{h}>\lambda_{c e l} \geq c_{l}
\end{aligned}
$$

\section{Comparative Static Analysis Calculations}

Since $n_{h, \text { in }}$ and $n_{l, i n}$ are discrete variables, we cannot look at derivatives. Hence, we look at differences. Now, we define the difference operator. The change in $S_{t}(., .,$.$) with respect to a unit$ change in $n_{t, i n}$ is denoted by $\frac{\triangle S_{t}\left(F, n_{h, i n}, n_{l, i n}\right)}{\triangle n_{t, i n}}$.

$$
S_{h}(C E A, i n)=\frac{n_{l}\left(r_{f}-r\right) w_{l}}{n_{h}} \text { if } c_{h}>\lambda_{\text {cea }} \geq c_{l}
$$

- Change in $S_{h}(C E A, i n)$ with respect to $n_{l}$, if $c_{h}>\lambda_{c e a} \geq c_{l}$

$$
\frac{\triangle S_{h}(C E A, i n)}{\triangle n_{l}}=\frac{w_{l}\left(r_{f}-r\right)}{n_{h}}<0
$$

- Change in $S_{h}(C E A, i n)$ with respect to $n_{h}$, if $c_{h}>\lambda_{c e a} \geq c_{l}$

$$
\frac{\triangle S_{h}(C E A, i n)}{\triangle n_{h}}=\frac{-n_{l} w_{l}\left(r_{f}-r\right)}{\left(n_{h}\right)^{2}} \text { or } \frac{-n_{l} w_{l}\left(r_{f}-r\right)}{\left(n_{h}+1\right) n_{h}}>0
$$

- Change in $S_{h}(C E A, i n)$ with respect to $w_{l}$, if $c_{h}>\lambda_{c e a} \geq c_{l}$

$$
\frac{\partial S_{h}(C E A, i n)}{\partial w_{l}}=\frac{n_{l}\left(r_{f}-r\right)}{n_{h}}<0
$$

- Change in $S_{h}(C E A, i n)$ with respect to $w_{h}$, if $c_{h}>\lambda_{\text {cea }} \geq c_{l}$

$$
\begin{aligned}
& \frac{\partial S_{h}(C E A, i n)}{\partial w_{h}}=0 \\
& \qquad S_{h}(C E A, i n)=\frac{n_{l}}{n_{h}+n_{l}}\left(1+r_{f}\right)\left(w_{l}-w_{h}\right) \text { if } c_{h}>c_{l} \geq \lambda_{\text {cea }}
\end{aligned}
$$


- Change in $S_{h}(C E A, i n)$ with respect to $n_{l}$, if $c_{h}>c_{l} \geq \lambda_{\text {cea }}$ $\frac{\triangle S_{h}(C E A, i n)}{\triangle n_{l}}=\frac{n_{h}\left(1+r_{f}\right)\left(w_{l}-w_{h}\right)}{\left(n_{h}+n_{l}\right)^{2}}$ or $\frac{n_{h}\left(1+r_{f}\right)\left(w_{l}-w_{h}\right)}{\left(n_{h}+n_{l}+1\right)\left(n_{h}+n_{l}\right)}<0$

- Change in $S_{h}(C E A, i n)$ with respect to $n_{h}$, if $c_{h}>c_{l} \geq \lambda_{c e a}$ $\frac{\triangle S_{h}(C E A, i n)}{\triangle n_{h}}=-\frac{n_{l}\left(1+r_{f}\right)\left(w_{l}-w_{h}\right)}{\left(n_{h}+n_{l}\right)^{2}}$ or $-\frac{n_{l}\left(1+r_{f}\right)\left(w_{l}-w_{h}\right)}{\left(n_{h}+n_{l}+1\right)\left(n_{h}+n_{l}\right)}>0$

- Change in $S_{h}(C E A, i n)$ with respect to $w_{l}$, if $c_{h}>c_{l} \geq \lambda_{\text {cea }}$ $\frac{\partial S_{h}(C E A, i n)}{\partial w_{l}}=\frac{n_{l}}{n_{h}+n_{l}}\left(1+r_{f}\right)>0$

- Change in $S_{h}(C E A, i n)$ with respect to $w_{h}$, if $c_{h}>c_{l} \geq \lambda_{c e a}$ $\frac{\partial S_{h}(C E A, i n)}{\partial w_{h}}=-\frac{n_{l}}{n_{h}+n_{l}}\left(1+r_{f}\right)<0$

$$
S_{l}(C E A, i n)=\left(r-r_{f}\right) w_{l} \text { if } c_{h}>\lambda_{c e a} \geq c_{l}
$$

- Change in $S_{l}(C E A, i n)$ with respect to $n_{l}$, if $c_{h}>\lambda_{c e a} \geq c_{l}$ $\frac{\triangle S_{l}(C E A, i n)}{\triangle n_{l}}=0$

- Change in $S_{l}(C E A, i n)$ with respect to $n_{h}$, if $c_{h}>\lambda_{c e a} \geq c_{l}$ $\frac{\triangle S_{l}(C E A, i n)}{\triangle n_{h}}=0$

- Change in $S_{l}(C E A, i n)$ with respect to $w_{l}$, if $c_{h}>\lambda_{c e a} \geq c_{l}$ $\frac{\partial S_{l}(C E A, i n)}{\partial w_{l}}=\left(r-r_{f}\right)>0$

- Change in $S_{l}(C E A, i n)$ with respect to $w_{h}$, if $c_{h}>\lambda_{c e a} \geq c_{l}$ $\frac{\partial S_{l}(C E A, i n)}{\partial w_{h}}=0$

$$
S_{l}(C E A, i n)=\frac{n_{h}}{n_{h}+n_{l}}\left(1+r_{f}\right)\left(w_{h}-w_{l}\right) \text { if } c_{h}>c_{l} \geq \lambda_{\text {cea }}
$$

- Change in $S_{l}(C E A, i n)$ with respect to $n_{l}$, if $c_{h}>c_{l} \geq \lambda_{c e a}$

$$
\frac{\triangle S_{l}(C E A, i n)}{\triangle n_{l}}=-\frac{n_{h}\left(1+r_{f}\right)\left(w_{h}-w_{l}\right)}{\left(n_{h}+n_{l}\right)^{2}} \text { or }-\frac{n_{h}\left(1+r_{f}\right)\left(w_{h}-w_{l}\right)}{\left(n_{h}+n_{l}+1\right)\left(n_{h}+n_{l}\right)}<0
$$

- Change in $S_{l}(C E A, i n)$ with respect to $n_{h}$, if $c_{h}>c_{l} \geq \lambda_{c e a}$

$$
\frac{\triangle S_{l}(C E A, i n)}{\triangle n_{h}}=\frac{n_{l}\left(1+r_{f}\right)\left(w_{h}-w_{l}\right)}{\left(n_{h}+n_{l}\right)^{2}} \text { or } \frac{n_{l}\left(1+r_{f}\right)\left(w_{h}-w_{l}\right)}{\left(n_{h}+n_{l}+1\right)\left(n_{h}+n_{l}\right)}>0
$$

- Change in $S_{l}(C E A, i n)$ with respect to $w_{l}$, if $c_{h}>c_{l} \geq \lambda_{c e a}$ $\frac{\partial S_{l}(C E A, i n)}{\partial w_{l}}=-\frac{n_{h}}{n_{h}+n_{l}}\left(1+r_{f}\right)<0$ 
- Change in $S_{l}(C E A, i n)$ with respect to $w_{h}$, if $c_{h}>c_{l} \geq \lambda_{c e a}$

$$
\begin{aligned}
\frac{\partial S_{l}(C E A, i n)}{\partial w_{h}}=\frac{n_{h}}{n_{h}+n_{l}}\left(1+r_{f}\right) & >0 \\
S_{l}(C E L, i n) & =\frac{n_{h}}{n_{h}+n_{l}}\left(r-r_{f}\right)\left(w_{l}-w_{h}\right) \text { if } c_{h}>c_{l}>\lambda_{c e l}
\end{aligned}
$$

- Change in $S_{l}(C E L, i n)$ with respect to $n_{l}$, if $c_{h}>c_{l}>\lambda_{c e l}$

$$
\frac{\triangle S_{l}(C E L, i n)}{\triangle n_{l}}=-\frac{n_{h}\left(w_{l}-w_{h}\right)\left(r-r_{f}\right)}{\left(n_{h}+n_{l}\right)^{2}} \text { or }-\frac{n_{h}\left(w_{l}-w_{h}\right)\left(r-r_{f}\right)}{\left(n_{h}+n_{l}+1\right)\left(n_{h}+n_{l}\right)}>0
$$

- Change in $S_{l}\left(C E L\right.$, in) with respect to $n_{h}$, if $c_{h}>c_{l}>\lambda_{c e l}$

$$
\frac{\triangle S_{l}(C E L, i n)}{\triangle n_{h}}=\frac{n_{l}\left(w_{l}-w_{h}\right)\left(r-r_{f}\right)}{\left(n_{h}+n_{l}\right)^{2}} \text { or } \frac{n_{l}\left(w_{l}-w_{h}\right)\left(r-r_{f}\right)}{\left(n_{h}+n_{l}+1\right)\left(n_{h}+n_{l}\right)}<0
$$

- Change in $S_{l}(C E L, i n)$ with respect to $w_{l}$, if $c_{h}>c_{l}>\lambda_{c e l}$

$$
\frac{\partial S_{l}(C E L, i n)}{\partial w_{l}}=\frac{n_{h}}{n_{h}+n_{l}}\left(r-r_{f}\right)>0
$$

- Change in $S_{l}(C E L, i n)$ with respect to $w_{h}$, if $c_{h}>c_{l}>\lambda_{c e l}$

$$
\frac{\partial S_{l}(C E L, i n)}{\partial w_{h}}=-\frac{n_{h}}{n_{h}+n_{l}}\left(r-r_{f}\right)<0
$$

$$
S_{l}(C E L, i n)=-\left(1+r_{f}\right) w_{l} \text { if } c_{h}>\lambda_{c e l} \geq c_{l}
$$

- Change in $S_{l}(C E L, i n)$ with respect to $n_{l}$, if $c_{h}>\lambda_{c e l} \geq c_{l}$

$$
\frac{\triangle S_{l}(C E L, i n)}{\triangle n_{l}}=0
$$

- Change in $S_{l}(C E L, i n)$ with respect to $n_{h}$, if $c_{h}>\lambda_{c e l} \geq c_{l}$

$$
\frac{\triangle S_{l}(C E L, i n)}{\triangle n_{h}}=0
$$

- Change in $S_{l}(C E L, i n)$ with respect to $w_{l}$, if $c_{h}>\lambda_{c e l} \geq c_{l}$ $\frac{\partial S_{l}(C E L, i n)}{\partial w_{l}}=-\left(1+r_{f}\right)<0$

- Change in $S_{l}(C E L, i n)$ with respect to $w_{h}$, if $c_{h}>\lambda_{c e l} \geq c_{l}$ $\frac{\partial S_{l}(C E L, i n)}{\partial w_{h}}=0$

$$
S_{h}(C E L, i n)=\frac{n_{l}}{n_{h}+n_{l}}\left(r-r_{f}\right)\left(w_{h}-w_{l}\right) \text { if } c_{h}>c_{l}>\lambda_{c e l}
$$

- Change in $S_{h}(C E L, i n)$ with respect to $n_{l}$, if $c_{h}>c_{l}>\lambda_{c e l}$

$$
\frac{\triangle S_{h}(C E L, i n)}{\triangle n_{l}}=\frac{n_{h}\left(w_{h}-w_{l}\right)\left(r-r_{f}\right)}{\left(n_{h}+n_{l}\right)^{2}} \text { or } \frac{n_{h}\left(w_{h}-w_{l}\right)\left(r-r_{f}\right)}{\left(n_{h}+n_{l}+1\right)\left(n_{h}+n_{l}\right)}>0
$$


- Change in $S_{h}(C E L$, in $)$ with respect to $n_{h}$, if $c_{h}>c_{l}>\lambda_{c e l}$

$\frac{\triangle S_{h}(C E L, i n)}{\triangle n_{h}}=-\frac{n_{l}\left(w_{h}-w_{l}\right)\left(r-r_{f}\right)}{\left(n_{h}+n_{l}\right)^{2}}$ or $-\frac{n_{l}\left(w_{h}-w_{l}\right)\left(r-r_{f}\right)}{\left(n_{h}+n_{l}+1\right)\left(n_{h}+n_{l}\right)}<0$

- Change in $S_{h}(C E L, i n)$ with respect to $w_{l}$, if $c_{h}>c_{l}>\lambda_{c e l}$

$\frac{\partial S_{h}(C E L, i n)}{\partial w_{l}}=-\frac{n_{l}}{n_{h}+n_{l}}\left(r-r_{f}\right)<0$

- Change in $S_{h}(C E L$, in $)$ with respect to $w_{h}$, if $c_{h}>c_{l}>\lambda_{c e l}$

$$
\begin{aligned}
& \frac{\partial S_{h}(C E L, i n)}{\partial w_{h}}=\frac{n_{l}}{n_{h}+n_{l}}\left(r-r_{f}\right)>0 \\
& \qquad S_{h}(C E L, i n)=\frac{n_{l}}{n_{h}}\left(1+r_{f}\right) w_{l} \text { if } c_{h}>\lambda_{c e l} \geq c_{l}
\end{aligned}
$$

- Change in $S_{h}(C E L, i n)$ with respect to $n_{l}$, if $c_{h}>\lambda_{c e l} \geq c_{l}$

$\frac{\triangle S_{h}(C E L, i n)}{\triangle n_{l}}=\frac{\left(1+r_{f}\right) w_{l}}{n_{h}}>0$

- Change in $S_{h}(C E L, i n)$ with respect to $n_{h}$, if $c_{h}>\lambda_{c e l} \geq c_{l}$

$\frac{\triangle S_{h}(C E L, i n)}{\triangle n_{h}}=-\frac{n_{l}\left(1+r_{f}\right) w_{l}}{\left(n_{h}\right)^{2}}$ or $-\frac{n_{l}\left(1+r_{f}\right) w_{l}}{\left(n_{h}+1\right)\left(n_{h}\right)}<0$

- Change in $S_{h}(C E L, i n)$ with respect to $w_{l}$, if $c_{h}>\lambda_{c e l} \geq c_{l}$

$\frac{\partial S_{h}(C E L, i n)}{\partial w_{l}}=\frac{n_{l}}{n_{h}}\left(1+r_{f}\right)>0$

- Change in $S_{h}(C E L, i n)$ with respect to $w_{h}$, if $c_{h}>\lambda_{c e l} \geq c_{l}$

$\frac{\partial S_{h}(C E L, i n)}{\partial w_{h}}=0$

\section{B - Proofs of Preparatory Results}

Proof of Lemma 1. (i) Assume that the number of claimants for whom $c_{j} \leq \lambda_{\text {cea }}$ is $k^{*}<n$. It immediately follows that for all $c_{j} \leq \lambda_{\text {cea }}, C E A_{j}(C, E)=c_{j}$. Therefore, for all $c_{j} \leq \lambda_{\text {cea }}$, since $P_{j}(C, E)<c_{j}$, we get $C E A_{j}(C, E)>P_{j}(C, E)$. We now analyze the case, $c_{j}>\lambda_{\text {cea }}$. For all such claimants, $C E A_{j}(C, E)=\lambda_{\text {cea }}$. This can be written as

$$
C E A_{j}(C, E)=\lambda_{\text {cea }}=\frac{E-\sum_{i=1}^{k^{*}} c_{i}}{n-k^{*}} .
$$

Furthermore, we know that

$$
P_{j}(C, E)=c_{j} \frac{E}{\sum_{i \in N} c_{i}} .
$$

To find out whether there exists $c^{*}$, which satisfies the statement in the lemma, we consider the equality,

$$
\frac{E-\sum_{i=1}^{k^{*}} c_{i}}{n-k^{*}}=c^{*} \frac{E}{\sum_{i \in N} c_{i}}
$$


This can be rewritten as

$$
c^{*}=\frac{\left(E-\sum_{i=1}^{k^{*}} c_{i}\right) \sum_{i \in N} c_{i}}{\left(n-k^{*}\right) E}=\lambda_{c e a} \frac{\sum_{i \in N} c_{i}}{E} .
$$

Since $\left(E-\sum_{i=1}^{k^{*}} c_{i}\right)>0, c^{*}>0$. Moreover, since

$$
\frac{\sum_{i \in N} c_{i}}{E}>1
$$

(by the definition of bankruptcy), we have $c^{*}>\lambda_{\text {cea }}$. Now, pick an agent $j$ such that $\lambda_{\text {cea }}<c_{j}$. Then,

$$
c_{j} \lesseqgtr c^{*} \Leftrightarrow P_{j}(C, E)=c_{j} \frac{E}{\sum_{i \in N} c_{i}} \lesseqgtr c^{*} \frac{E}{\sum_{i \in N} c_{i}} .
$$

Using (14), we can rewrite this as

$$
c_{j} \lesseqgtr c^{*} \Leftrightarrow P_{j}(C, E)=c_{j} \frac{E}{\sum_{i \in N} c_{i}} \lesseqgtr \lambda_{c e a} \frac{\sum_{i \in N} c_{i}}{E} \frac{E}{\sum_{i \in N} c_{i}}=\lambda_{c e a}=C E A_{j}(C, E) .
$$

Thus, the result follows.

(ii) Assume that the number of claimants for whom $c_{j} \leq \lambda_{\text {cel }}$ is $\widetilde{k}<n$. It immediately follows that for all $c_{j} \leq \lambda_{\text {cel }}, C E L_{j}(C, E)=0$. Therefore, for all $c_{j} \leq \lambda_{\text {cel }}$, since $0<P_{j}(C, E)$, we get $C E L_{j}(C, E)<P_{j}(C, E)$. We now analyze the case $c_{j}>\lambda_{c e l}$. For all such claimants, $C E L_{j}(C, E)=$ $c_{j}-\lambda_{c e l}>0$, where $\lambda_{c e l}$ can be written as

$$
\lambda_{c e l}=\frac{\sum_{i=\widetilde{k}+1}^{n} c_{i}-E}{n-\widetilde{k}} .
$$

Therefore, for all $c_{j}>\lambda_{c e l}, C E L_{j}(C, E)$ can be written as

$$
C E L_{j}(C, E)=c_{j}-\frac{\sum_{i=\widetilde{k}+1}^{n} c_{i}-E}{n-\widetilde{k}} .
$$

Furthermore, we know that

$$
P_{j}(C, E)=c_{j} \frac{E}{\sum_{i \in N} c_{i}} .
$$

To find out whether there exists $\widetilde{c}$, which satisfies the statement in the lemma, we consider the equality,

$$
\widetilde{c}-\frac{\sum_{i=\widetilde{k}+1}^{n} c_{i}-E}{n-\widetilde{k}}=\widetilde{c} \frac{E}{\sum_{i \in N} c_{i}} .
$$

This can be rewritten as

$$
\widetilde{c}=\frac{\left(\sum_{i=\widetilde{k}+1}^{n} c_{i}-E\right) \sum_{i \in N} c_{i}}{(n-\widetilde{k})\left(\sum_{i \in N} c_{i}-E\right)}=\lambda_{c e l} \frac{\sum_{i \in N} c_{i}}{\sum_{i \in N} c_{i}-E}>0 .
$$

Moreover, since

$$
\frac{\sum_{i \in N} c_{i}}{\sum_{i \in N} c_{i}-E}>1
$$


we have $\widetilde{c}>\lambda_{c e l}$. Now pick an agent $j$ such that $c_{j}>\lambda_{c e l}$. Then,

$$
c_{j} \lesseqgtr \widetilde{c} \Leftrightarrow P_{j}(C, E)=c_{j} \frac{E}{\sum_{i \in N} c_{i}} \lesseqgtr \widetilde{c} \frac{E}{\sum_{i \in N} c_{i}} .
$$

Using (15), we can rewrite this as

$$
c_{j} \lesseqgtr \widetilde{c} \Leftrightarrow P_{j}(C, E)=c_{j} \frac{E}{\sum_{i \in N} c_{i}} \lesseqgtr \lambda_{c e l} \frac{\sum_{i \in N} c_{i}}{\sum_{i \in N} c_{i}-E} \frac{E}{\sum_{i \in N} c_{i}}=\lambda_{c e l} \frac{E}{\sum_{i \in N} c_{i}-E} .
$$

We know that an agent $j$ with a claim $c_{j}>\lambda_{\text {cel }}$ receives a payoff, $C E L_{j}(C, E)=c_{j}-\lambda_{c e l}$ and at $c_{j}=\widetilde{c}$,

$$
C E L_{j}(C, E)=c_{j}-\lambda_{c e l}=\lambda_{c e l} \frac{E}{\sum_{i \in N} c_{i}-E}=P_{j}(C, E) .
$$

Moreover, at $c_{j}=\lambda_{c e l}$

$$
P_{j}(C, E)=\lambda_{c e l} \frac{E}{\sum_{i \in N} c_{i}}>0=C E L_{j}(C, E) .
$$

Therefore, these two linear functions (i.e., $P_{j}(C, E)=c_{j}\left(E / \sum_{i \in N} c_{i}\right)$ and $\left.C E L_{j}(C, E)=c_{j}-\lambda_{c e l}\right)$ take different values at $c_{j}=\lambda_{c e l}$ and the same value at $c_{j}=\widetilde{c}$. In particular, $P_{j}(C, E)$ takes a higher value at $c_{j}=\lambda_{c e l}$. Therefore, $c_{j} \lesseqgtr \widetilde{c}$ implies

$$
P_{j}(C, E)=c_{j} \frac{E}{\sum_{i \in N} c_{i}} \gtreqless C E L_{j}(C, E) .
$$

Hence, $\widetilde{c}$ satisfies the statement in (ii).

Proof of Lemma 2. Under $C E A$, there are two cases: (a-1) $c_{h}>c_{l}>\lambda_{\text {cea }}$ and (a-2) $c_{h}>\lambda_{\text {cea }} \geq$ $c_{l}$.

(a-1) If $c_{h}>c_{l} \geq \lambda_{\text {cea }}$, then $C E A$ allocates the estate equally. Hence, $C E A_{l}(C, E)=$ $C E A_{h}(C, E)=\frac{E}{n_{h}+n_{l}}$. Therefore,

$$
S_{l}(C, E, C E A)=\left(\frac{E}{n}-\frac{E}{n_{h} c_{h}+n_{l} c_{l}} c_{l}\right),
$$

which satisfies

$$
S_{l}(C, E, C E A)=\frac{E n_{h}\left[c_{h}-c_{l}\right]}{\left[n_{h}+n_{l}\right]\left[n_{h} c_{h}+n_{l} c_{l}\right]}>0 .
$$

Similarly, $S_{h}(C, E, C E A)$ can be written as

$$
S_{h}(C, E, C E A)=\left(\frac{E}{n}-\frac{E}{n_{h} c_{h}+n_{l} c_{l}} c_{h}\right)
$$

which satisfies

$$
S_{h}(C, E, C E A)=\frac{E n_{l}\left[c_{l}-c_{h}\right]}{\left[n_{h}+n_{l}\right]\left[n_{h} c_{h}+n_{l} c_{l}\right]}<0 .
$$

(a-2) If $c_{h}>\lambda_{c e a} \geq c_{l}$, then for each agent with claim $c_{l}, C E A_{l}(C, E)=c_{l}$ and for each agent with claim $c_{h}, C E A_{h}(C, E)=\frac{E-n_{l} c_{l}}{n_{h}}$. Therefore,

$$
S_{l}(C, E, C E A)=c_{l}\left(1-\frac{E}{n_{h} c_{h}+n_{l} c_{l}}\right),
$$


which satisfies

$$
S_{l}(C, E, C E A)=\frac{c_{l}\left[n_{h} c_{h}+n_{l} c_{l}-E\right]}{n_{h} c_{h}+n_{l} c_{l}}>0 .
$$

Similarly, $S_{h}(C, E, C E A)$ can be written as

$$
S_{h}(C, E, C E A)=\left(\frac{E-n_{l} c_{l}}{n_{h}}-\frac{E}{n_{h} c_{h}+n_{l} c_{l}}\right),
$$

which satisfies

$$
S_{h}(C, E, C E A)=\frac{n_{l} c_{l}\left[E-n_{h} c_{h}-n_{l} c_{l}\right]}{n_{h}\left[n_{h} c_{h}+n_{l} c_{l}\right]}<0 .
$$

Under $C E L$, there are two cases: (b-1) $c_{h}>c_{l}>\lambda_{\text {cel }}$ and (b-2) $c_{h}>\lambda_{c e l} \geq c_{l}$.

(b-1) If $c_{h}>c_{l}>\lambda_{\text {cel }}$, then $C E L$ allocates losses equally. Hence, for each agent with claim $c_{l}, C E L_{l}(C, E)=c_{l}-\left(\frac{n_{h} c_{h}+n_{l} c_{l}-E}{n_{h}+n_{l}}\right)$ and for each agent with claim $c_{h}, C E L_{h}(C, E)=$ $c_{h}-\left(\frac{n_{h} c_{h}+n_{l} c_{l}-E}{n_{h}+n_{l}}\right)$. Therefore,

$$
S_{l}(C, E, C E L)=\left[c_{l}-\left(\frac{n_{h} c_{h}+n_{l} c_{l}-E}{n_{h}+n_{l}}\right)-\frac{E}{n_{h} c_{h}+n_{l} c_{l}} c_{l}\right],
$$

which satisfies

$$
S_{l}(C, E, C E L)=\frac{n_{h}\left[c_{l}-c_{h}\right]\left[n_{h} c_{h}+n_{l} c_{l}-E\right]}{\left[n_{h}+n_{l}\right]\left[n_{h} c_{h}+n_{l} c_{l}\right]}<0 .
$$

Similarly, $S_{h}(C, E, C E L)$ can be written as

$$
S_{h}(C, E, C E L)=\left[c_{h}-\left(\frac{n_{h} c_{h}+n_{l} c_{l}-E}{n_{h}+n_{l}}\right)-\frac{E}{n_{h} c_{h}+n_{l} c_{l}} c_{h}\right]
$$

which satisfies

$$
S_{h}(C, E, C E L)=\frac{n_{l}\left[c_{h}-c_{l}\right]\left[n_{h} c_{h}+n_{l} c_{l}-E\right]}{\left[n_{h}+n_{l}\right]\left[n_{h} c_{h}+n_{l} c_{l}\right]}>0 .
$$

(b-2) If $c_{h}>\lambda_{c e l} \geq c_{l}$, then for each agent with claim $c_{l}, C E L_{l}(C, E)=0$ and for each agent with claim $c_{h}, C E L_{h}(C, E)=\frac{E}{n_{h}}$. Therefore,

$$
S_{l}(C, E, C E L)=-\frac{E}{n_{h} c_{h}+n_{l} c_{l}} c_{l}<0
$$

and

$$
S_{h}(C, E, C E L)=\frac{n_{l}}{n_{h}} \frac{E c_{l}}{n_{h} c_{h}+n_{l} c_{l}}>0 .
$$

Proof of Lemma 3. Below, we plug into $V_{j, i n}\left(P, n_{h, i n}, n_{l, i n}\right)=c_{j, i n} \frac{E}{\sum_{i \in N_{l} \cup N_{h}} c_{i, i n}}$,

$$
\begin{aligned}
\left(1+r_{f}\right) \sum_{i \in N_{l} \cup N_{h}} w_{i, i n} & \text { for } E \\
(1+r) \sum_{i \in N_{l} \cup N_{h}} w_{i, i n} & \text { for } \sum_{i \in N_{l} \cup N_{h}} c_{i, i n} \text {, and } \\
(1+r) w_{j} & \text { for } \quad c_{j} .
\end{aligned}
$$


Hence,

$$
\begin{aligned}
V_{j, i n}\left(P, n_{h, i n}, n_{l, i n}\right) & =(1+r) w_{j} \frac{\left(1+r_{f}\right) \sum_{i \in N_{l} \cup N_{h}} w_{i, i n}}{(1+r) \sum_{i \in N_{l} \cup N_{h}} w_{i, i n}}, \\
& =\left(1+r_{f}\right) w_{j}=P_{j} .
\end{aligned}
$$

Proof of Lemma 4. (i) Pick any $n_{n, i n}>0$ and $n_{l, i n}>0$. We know by Corollary 2 that

$$
\begin{aligned}
S_{l}\left(C E A, n_{h, i n}, n_{l, i n}\right) & >0 \text { and } \\
S_{h}\left(C E A, n_{h, i n}, n_{l, i n}\right) & <0 .
\end{aligned}
$$

Therefore, by the definition of $c^{*}$ (see Lemma 1), $c_{l} \leq c^{*} \leq c_{h}$.

(ii) Pick any $n_{n, i n}>0$ and $n_{l, i n}>0$. We know by Corollary 2 that

$$
\begin{aligned}
S_{h}\left(C E L, n_{h, i n}, n_{l, i n}\right) & >0 \text { and } \\
S_{l}\left(C E L, n_{h, i n}, n_{l, i n}\right) & <0 .
\end{aligned}
$$

Therefore, by the definition of $\widetilde{c}$ (see Lemma 1), $c_{l} \leq \widetilde{c} \leq c_{h}$.

Proof of Lemma 5. (i) There are two possible cases for $C E A$ : (a-1) $c_{h}>c_{l}>\lambda_{\text {cea }}$ and (a-2) $c_{h}>\lambda_{\text {cea }} \geq c_{l}$. We know from Corollary 2 (a-1),

$$
S_{l}\left(C E A, n_{h, i n}, n_{l, i n}\right)=\frac{n_{h, i n}}{n_{h, i n}+n_{l, i n}}\left(1+r_{f}\right)\left(w_{h}-w_{l}\right) .
$$

Thus,

$$
\frac{\triangle S_{l}\left(C E A, n_{h, i n}, n_{l, i n}\right)}{\triangle n_{l, i n}}=-\frac{n_{h, i n}}{\left(n_{h, i n}+n_{l, i n}+1\right)\left(n_{h, i n}+n_{l, i n}\right)}\left(1+r_{f}\right)\left(w_{h}-w_{l}\right)<0 .
$$

On the other hand, in Corollary 2 (a-2),

$$
S_{l}\left(C E A, n_{h, i n}, n_{l, i n}\right)=\left(r-r_{f}\right) w_{l} .
$$

Thus,

$$
\frac{\triangle S_{l}\left(C E L, n_{h, i n}, n_{l, i n}\right)}{\triangle n_{l, i n}}=0 .
$$

(ii) There are two possible cases for $C E A$ : (a-1) and (a-2). We know from Corollary 2 (a-1),

$$
S_{h}\left(C E A, n_{h, i n}, n_{l, i n}\right)=\frac{n_{l, i n}}{n_{h, i n}+n_{l, i n}}\left(1+r_{f}\right)\left(w_{l}-w_{h}\right) .
$$

Thus,

$$
\frac{\triangle S_{h}\left(C E A, n_{h, i n}, n_{l, i n}\right)}{\triangle n_{l, i n}}=\frac{n_{h, i n}\left(1+r_{f}\right)\left(w_{l}-w_{h}\right)}{\left(n_{h, i n}+n_{l, i n}+1\right)\left(n_{h, i n}+n_{l, i n}\right)}<0 .
$$


On the other hand, in Corollary 2 (a-2),

$$
S_{h}\left(C E A, n_{h, i n}, n_{l, i n}\right)=\frac{n_{l, i n}}{n_{h, i n}}\left(r_{f}-r\right) w_{l} .
$$

Thus,

$$
\frac{\triangle S_{h}\left(C E A, n_{h, i n}, n_{l, i n}\right)}{\triangle n_{l, i n}}=\frac{\left(r_{f}-r\right) w_{l}}{n_{h, i n}}<0 .
$$

(iii) There are two possible cases for $C E L$ : (b-1) $c_{h}>c_{l}>\lambda_{\text {cel }}$ and (b-2) $c_{h}>\lambda_{c e l} \geq c_{l}$. We know from Corollary 2 (b-1),

$$
S_{l}\left(C E L, n_{h, i n}, n_{l, i n}\right)=\frac{n_{h, i n}}{n_{h, i n}+n_{l, i n}}\left(r-r_{f}\right)\left(w_{l}-w_{h}\right) .
$$

Thus,

$$
\frac{\triangle S_{l}\left(C E L, n_{h, i n}, n_{l, i n}\right)}{\triangle n_{h, \text { in }}}=\frac{n_{l, i n}}{\left(n_{h, i n}+n_{l, i n}+1\right)\left(n_{h, i n}+n_{l, i n}\right)}\left(r-r_{f}\right)\left(w_{l}-w_{h}\right)<0 .
$$

On the other hand, in Corollary 2 (b-2),

$$
S_{l}\left(C E L, n_{h, i n}, n_{l, i n}\right)=-\left(1+r_{f}\right) w_{l} .
$$

Thus,

$$
\frac{\triangle S_{l}\left(C E L, n_{h, i n}, n_{l, i n}\right)}{\triangle n_{h, i n}}=0 .
$$

(iv) There are two possible cases for $C E L$ : (b-1) and (b-2). We know from Corollary 2 (b-1),

$$
S_{h}\left(C E L, n_{h, i n}, n_{l, i n}\right)=\frac{n_{l, i n}}{n_{h, i n}+n_{l, i n}}\left[\left(r-r_{f}\right)\left(w_{h}-w_{l}\right)\right] .
$$

Thus,

$$
\frac{\triangle S_{h}\left(C E L, n_{h, i n}, n_{l, i n}\right)}{\triangle n_{h, i n}}=-\frac{n_{l, i n}}{\left(n_{h, i n}+n_{l, i n}+1\right)\left(n_{h, i n}+n_{l, i n}\right)}\left(r-r_{f}\right)\left(w_{h}-w_{l}\right)<0 .
$$

On the other hand, in Corollary 2 (b-2),

$$
S_{h}\left(C E L, n_{h, i n}, n_{l, i n}\right)=\frac{n_{l, i n}}{n_{h, i n}}\left(1+r_{f}\right) w_{l} .
$$

Thus,

$$
\frac{\triangle S_{h}\left(C E L, n_{h, i n}, n_{l, i n}\right)}{\triangle n_{h, i n}}=-\frac{n_{l, i n}}{\left(n_{h, i n}+1\right)\left(n_{h, i n}\right)}\left(1+r_{f}\right) w_{l}<0 .
$$

(v) There are two possible cases for $C E A$ : (a-1) $c_{h}>c_{l}>\lambda_{\text {cea }}$ and (a-2) $c_{h}>\lambda_{c e a} \geq c_{l}$. We know from Corollary 2 (a-1),

$$
S_{l}\left(C E A, n_{h, i n}, n_{l, i n}\right)=\frac{n_{h, i n}}{n_{h, i n}+n_{l, i n}}\left(1+r_{f}\right)\left(w_{h}-w_{l}\right) .
$$


Thus,

$$
\frac{\triangle S_{l}\left(C E A, n_{h, i n}, n_{l, i n}\right)}{\triangle n_{h, i n}}=\frac{n_{l, i n}}{\left(n_{h, i n}+n_{l, i n}+1\right)\left(n_{h, i n}+n_{l, i n}\right)}\left(1+r_{f}\right)\left(w_{h}-w_{l}\right)>0 .
$$

On the other hand, in Corollary 2 (a-2),

$$
S_{l}\left(C E A, n_{h, i n}, n_{l, i n}\right)=\left(r-r_{f}\right) w_{l} .
$$

Thus,

$$
\frac{\triangle S_{l}\left(C E L, n_{h, i n}, n_{l, i n}\right)}{\triangle n_{l, i n}}=0 .
$$

(vi) There are two possible cases for $C E A$ : (a-1) and (a-2). We know from Corollary 2 (a-1),

$$
S_{h}\left(C E A, n_{h, i n}, n_{l, i n}\right)=\frac{n_{l, i n}}{n_{h, i n}+n_{l, i n}}\left(1+r_{f}\right)\left(w_{l}-w_{h}\right)<0 .
$$

Thus,

$$
\frac{\triangle S_{h}\left(C E A, n_{h, i n}, n_{l, i n}\right)}{\triangle n_{h, i n}}=-\frac{n_{l, i n}}{\left(n_{h, i n}+n_{l, i n}+1\right)\left(n_{h, i n}+n_{l, i n}\right)}\left(1+r_{f}\right)\left(w_{l}-w_{h}\right)>0 .
$$

On the other hand, in Corollary 2 (a-2),

$$
S_{h}\left(C E A, n_{h, i n}, n_{l, i n}\right)=\frac{n_{l, i n}}{n_{h, i n}}\left(r_{f}-r\right) w_{l}<0 .
$$

Thus,

$$
\frac{\triangle S_{h}\left(C E A, n_{h, i n}, n_{l, i n}\right)}{\triangle n_{h, i n}}=-\frac{n_{l, i n}}{n_{h, i n}\left(n_{h, i n}+1\right)}\left(r_{f}-r\right) w_{l}>0 .
$$

(vii) There are two possible cases for $C E L$ : (b-1) $c_{h}>c_{l}>\lambda_{\text {cel }}$ and (b-2) $c_{h}>\lambda_{c e l} \geq c_{l}$. We know from Corollary 2 (b-1),

$$
S_{l}\left(C E L, n_{h, i n}, n_{l, i n}\right)=\frac{n_{h, i n}}{n_{h, i n}+n_{l, i n}}\left(r-r_{f}\right)\left(w_{l}-w_{h}\right)<0 .
$$

Thus,

$$
\frac{\triangle S_{l}\left(C E L, n_{h, i n}, n_{l, i n}\right)}{\triangle n_{l, i n}}=-\frac{n_{h, i n}}{\left(n_{h, i n}+n_{l, i n}+1\right)\left(n_{h, i n}+n_{l, i n}\right)}\left(r-r_{f}\right)\left(w_{l}-w_{h}\right)>0 .
$$

On the other hand, in Corollary 2 (b-2),

$$
S_{l}\left(C E L, n_{h, i n}, n_{l, i n}\right)=-\left(1+r_{f}\right) w_{l}<0 .
$$

Therefore,

$$
\frac{\triangle S_{l}\left(C E L, n_{h, i n}, n_{l, i n}\right)}{\triangle n_{l, i n}}=0 .
$$

(viii) There are two possible cases for $C E L$ : (b-1) and (b-2). We know from Corollary 2 (b-1),

$$
S_{h}\left(C E L, n_{h, i n}, n_{l, i n}\right)=\frac{n_{l, i n}}{n_{h, i n}+n_{l, i n}}\left[\left(r-r_{f}\right)\left(w_{h}-w_{l}\right)\right]>0 .
$$


Thus,

$$
\frac{\triangle S_{h}\left(C E L, n_{h, i n}, n_{l, i n}\right)}{\triangle n_{l, i n}}=\frac{n_{h, i n}}{\left(n_{h, i n}+n_{l, i n}+1\right)\left(n_{h, i n}+n_{l, i n}\right)}\left[\left(r-r_{f}\right)\left(w_{h}-w_{l}\right)\right]>0 .
$$

On the other hand, in Corollary 2 (b-2),

$$
S_{h}\left(C E L, n_{h, i n}, n_{l, i n}\right)=\frac{n_{l, i n}}{n_{h, i n}}\left(1+r_{f}\right) w_{l} .
$$

Thus,

$$
\frac{\triangle S_{h}\left(C E L, n_{h, i n}, n_{l, i n}\right)}{\triangle n_{l, i n}}=\frac{\left(n_{l, i n}+1\right)}{n_{h, i n}}\left(1+r_{f}\right) w_{l}>0 .
$$

Proof of Lemma 6. We know that $P_{t}=\left(1+r_{f}\right) w_{t}$ and $V_{t}=(1+\bar{r}) w_{t}$. The expected payoff of a type $t$ agent under $P$ can be written as

$$
P_{i}^{e}=\pi_{s}(1+r) w_{t}+\left(1-\pi_{s}\right)\left(1+r_{f}\right) w_{t} .
$$

Therefore, a type $t$ agent chooses to play in under $P$ if

$$
P_{i}^{e}=\pi_{s}(1+r) w_{t}+\left(1-\pi_{s}\right)\left(1+r_{f}\right) w_{t} \geq(1+\bar{r}) w_{t}=V_{i, \text { out }} .
$$

This condition can be rewritten as

$$
\pi_{s}(1+r)+\left(1-\pi_{s}\right)\left(1+r_{f}\right) \geq(1+\bar{r}) .
$$

Since there are no income parameters in this inequality, it implies that for any two agents of different types, the condition determining their strategies under $P$ is identical, i.e.,

$$
P_{h}^{e} \geq V_{h, \text { out }} \Leftrightarrow P_{l}^{e} \geq V_{l, \text { out }} .
$$

Proof of Lemma 7. We analyze $P, C E A$, and $C E L$ separately since the argumentation for each case will be slightly different.

(P) Suppose, by a contradiction, that two agents $i$ and $j$ of type $t$ play different strategies in equilibrium. By Lemma $3, P_{i}=\left(1+r_{f}\right) w_{i}$ in case of bankruptcy. On the other hand, agent $i$ 's payoff is $(1+r) w_{i}$ in case of successful completion of the project and the outside option pays $(1+\bar{r}) w_{i}$. Since for all $i, j \in N_{t}$,

$$
\begin{aligned}
& P_{i}^{e}=\pi_{s}\left[(1+r) w_{t}\right]+\left(1-\pi_{s}\right)\left[\left(1+r_{f}\right) w_{t}\right] \text { and } \\
& P_{j}^{e}=\pi_{s}\left[(1+r) w_{t}\right]+\left(1-\pi_{s}\right)\left[\left(1+r_{f}\right) w_{t}\right],
\end{aligned}
$$

we have

$$
\begin{aligned}
& \pi_{s}\left[(1+r) w_{t}\right]+\left(1-\pi_{s}\right)\left[\left(1+r_{f}\right) w_{t}\right] \geq(1+\bar{r}) w_{t} \Leftrightarrow \\
& \pi_{s}\left[(1+r) w_{t}\right]+\left(1-\pi_{s}\right)\left[\left(1+r_{f}\right) w_{t}\right] \geq(1+\bar{r}) w_{t} .
\end{aligned}
$$


However, this logical statement implies that agents $i$ and $j$ cannot have different strategies in equilibrium; a contradiction. Hence the result follows.

The proofs for $C E A$ and $C E L$ require a separate analysis for $l$ type agents and $h$ type agents, since these agents are treated differently by $C E A$ and $C E L$.

(CEA) Suppose, by contradiction, that in equilibrium there exists an agent $i \in N_{h}$ who plays in whereas another agent $j \in N_{h}$ plays out under $C E A$. We denote the number of type $h$ agents playing in as $n_{h, i n}$ and the number of type $l$ agents playing $i n$ as $n_{l, i n}$. We consider two cases: (CEA - i) there is at least one agent $k \in N_{l}$ who plays in and (CEA - ii) there is no agent $k \in N_{l}$ who plays in.

(CEA - i) If there is at least one agent $k \in N_{l}$ who plays $i n$, i.e., $n_{l, i n} \geq 1$, by Corollary 2 ,

$$
\begin{aligned}
& \text { if } c_{h}>c_{l}>\lambda_{\text {cea }} \text {, then } S_{h}(C E A, \text { in })=\frac{n_{l}}{n_{h}+n_{l}}\left[\left(1+r_{f}\right)\left(w_{l}-w_{h}\right)\right]<0 \text { and } \\
& \text { if } c_{h}>\lambda_{\text {cea }} \geq c_{l} \text {, then } S_{h}(C E A, i n)=\frac{n_{l}\left(r_{f}-r\right) w_{l}}{n_{h}}<0 .
\end{aligned}
$$

Hence, we know that $S_{i}\left(C E A, n_{h, i n}, n_{l, i n}\right)<0$. Also, the equilibrium property and our tie-breaking assumption imply that

$$
V_{i, i n}^{e}\left(C E A, n_{h, \text { in }}, n_{l, i n}\right)=P_{i}^{e}+\left(1-\pi_{s}\right) S_{i}\left(C E A, n_{h, \text { in }}, n_{l, \text { in }}\right) \geq V_{i, \text { out }}
$$

and

$$
V_{j, \text { in }}^{e}\left(C E A, n_{h, \text { in }}+1, n_{l, \text { in }}\right)=P_{j}^{e}+\left(1-\pi_{s}\right) S_{j}\left(C E A, n_{h, \text { in }}+1, n_{l, \text { in }}\right)<V_{j, \text { out }} .
$$

Now, assume that player $j$ switches to $i n$. Then, number of type $h$ agents playing in is $n_{h, i n}+1$. The definition of $C E A$, the equilibrium property, and our tie-breaking assumption imply that

$$
V_{i, \text { in }}^{e}\left(C E A, n_{h, \text { in }}+1, n_{l, i n}\right)=V_{j, i n}^{e}\left(C E A, n_{h, \text { in }}+1, n_{l, i n}\right)<V_{j, \text { out }} \equiv V_{i, \text { out }} .
$$

On the other hand, by Corollary 2 and Lemma 5,

$$
S_{i}\left(C E A, n_{h, i n}, n_{l, i n}\right)<S_{i}\left(C E A, n_{h, i n}+1, n_{l, i n}\right)<0 .
$$

Hence,

$$
V_{i, i n}^{e}\left(C E A, n_{h, i n}, n_{l, i n}\right)<V_{i, i n}^{e}\left(C E A, n_{h, i n}+1, n_{l, i n}\right) .
$$

Therefore,

$$
\begin{aligned}
V_{i, \text { in }}^{e}\left(C E A, n_{h, \text { in }}, n_{l, i n}\right) & <V_{i, \text { in }}^{e}\left(C E A, n_{h, \text { in }}+1, n_{l, \text { in }}\right) \leq V_{i, \text { out }} \\
\Rightarrow V_{i, \text { in }}^{e}\left(C E A, n_{h, \text { in }}, n_{l, \text { in }}\right) & <V_{i, \text { out }} .
\end{aligned}
$$

However, if the above inequality is satisfied, agent $i$ playing in at the beginning cannot be an optimal action for him; a contradiction. Hence the result follows.

(CEA - ii) If there is no agent $k \in N_{l}$ who plays in under $C E A$ in equilibrium, i.e., $n_{l, \text { in }}=0$, this implies

$$
\begin{aligned}
S_{i}\left(C E A, n_{h, i n}, n_{l, i n}\right) & =0 \text { and } \\
V_{i, i n}^{e}\left(C E A, n_{h, i n}, n_{l, i n}\right) & =P_{i}^{e} .
\end{aligned}
$$


However, this implies that $P_{i}^{e} \geq V_{i, o u t}$. Hence, from (P) the result follows.

Now, suppose that in equilibrium there exists an agent $i \in N_{l}$ who plays $i n$ whereas another agent $j \in N_{l}$ plays out under $C E A$. We consider two cases: (CEA - iii) there is at least one agent $k \in N_{h}$ who plays in and (CEA - iv) there is no agent $k \in N_{h}$ who plays $i n$.

(CEA - iii) If there is at least one agent $k \in N_{h}$ who plays $i n$, i.e., $n_{h, i n} \geq 1$, by Corollary 2 ,

$$
\begin{aligned}
& \text { if } c_{h}>c_{l}>\lambda_{\text {cea }} \text {, then } S_{h}(C E A, i n)=\frac{n_{l}}{n_{h}+n_{l}}\left[\left(1+r_{f}\right)\left(w_{l}-w_{h}\right)\right]<0 \text { and } \\
& \text { if } c_{h}>\lambda_{\text {cea }} \geq c_{l} \text {, then } S_{h}(C E A, i n)=\frac{n_{l}\left(r_{f}-r\right) w_{l}}{n_{h}}<0 .
\end{aligned}
$$

Hence, we know that

$$
\begin{aligned}
S_{k}\left(C E A, n_{h, \text { in }}, n_{l, i n}\right) & <0 \text { and } \\
V_{k, \text { in }}^{e}\left(C E A, n_{h, \text { in }}, n_{l, \text { in }}\right) & =P_{k}^{e}+\left(1-\pi_{s}\right) S_{k}\left(C E A, n_{h, \text { in }}, n_{l, \text { in }}\right) \geq V_{k, \text { out }} .
\end{aligned}
$$

Now, assume that agent $j$ switches to playing in. The definition of $C E A$, the equilibrium property, and our tie-breaking assumption imply that

$$
V_{i, \text { in }}^{e}\left(C E A, n_{h, \text { in }}, n_{l, \text { in }}+1\right)=V_{j, \text { in }}^{e}\left(C E A, n_{h, \text { in }}, n_{l, \text { in }}+1\right)<V_{j, \text { out }} \equiv V_{i, \text { out }}
$$

where,

$$
\begin{aligned}
V_{j, i n}^{e}\left(C E A, n_{h, i n}, n_{l, i n}+1\right) & =P_{j}^{e}+\left(1-\pi_{s}\right) S_{j}\left(C E A, n_{h, i n}, n_{l, i n}+1\right) \text { and } \\
S_{j}\left(C E A, n_{h, i n}, n_{l, i n}+1\right) & >0 .
\end{aligned}
$$

However, if

$$
V_{k, i n}^{e}\left(C E A, n_{h, i n}, n_{l, i n}\right)=P_{k}^{e}+\left(1-\pi_{s}\right) S_{k}\left(C E A, n_{h, i n}, n_{l, i n}\right) \geq V_{k, \text { out }},
$$

then by proportionality,

$$
P_{k}^{e} \geq V_{k, \text { out }} \Leftrightarrow P_{j}^{e} \geq V_{j, \text { out }}
$$

and by Corollary 2 and Lemma 5

$$
S_{k}\left(C E A, n_{h, i n}, n_{l, i n}\right)<0 \text { and } S_{j}\left(C E A, n_{h, i n}, n_{l, i n}+1\right)>0
$$

leads to

$$
V_{j, \text { in }}^{e}\left(C E A, n_{h, \text { in }}, n_{l, \text { in }}+1\right)=V_{i, \text { in }}^{e}\left(C E A, n_{h, \text { in }}, n_{l, \text { in }}+1\right) \leq V_{j, \text { out }},
$$

which is a contradiction. Thus, in equilibrium if it is an optimal action for a type $h$ agent to play in under $C E A$, it cannot be an optimal action for any type $l$ agent to play out, since any type $l$ agent will have non-negative transfers. Hence, the result follows.

(CEA - iv) If there is no agent $k \in N_{h}$ who plays in under $C E A$ in equilibrium, i.e., $n_{h, i n}=0$, this implies

$$
\begin{aligned}
S_{i}\left(C E A, n_{h, i n}, n_{l, i n}\right) & =0 \text { and } \\
V_{i, i n}^{e}\left(C E A, n_{h, i n}, n_{l, i n}\right) & =P_{i}^{e} .
\end{aligned}
$$


Then, if agent $j$ switches to playing in, by Corollary 2 and Lemma 5 ,

$$
S_{k}\left(C E A, n_{h, i n}, n_{l, i n}\right)>S_{k}\left(C E A, n_{h, \text { in }}, n_{l, i n}+1\right) .
$$

Then, there will still be no agent $k \in N_{h}$ who plays $i n$. Hence, expected payoffs of type $l$ agents will not be affected by the existence of other type $l$ agents playing $i n$, which implies

$$
\begin{aligned}
V_{i, \text { in }}^{e}\left(C E A, n_{h, \text { in }}, n_{l, i n}\right) & =V_{i, \text { in }}^{e}\left(C E A, n_{h, \text { in }}, n_{l, i n}+1\right) \\
& =V_{j, \text { in }}^{e}\left(C E A, n_{h, \text { in }}, n_{l, \text { in }}+1\right) \\
\Rightarrow V_{i, \text { in }}^{e}\left(C E A, n_{h, \text { in }}, n_{l, i n}\right) & <V_{i, \text { out }} .
\end{aligned}
$$

If the above inequality is satisfied, playing in at the beginning cannot be an optimal action for agent $i$; a contradiction. Hence the result follows.

(CEL) The proof is similar to the proof for $C E A$. Suppose, by contradiction, that in equilibrium there exists an agent $i \in N_{l}$ who plays in whereas another agent $j \in N_{l}$ plays out under $C E L$. We denote the number of type $h$ agents playing in as $n_{h, \text { in }}$ and the number of type $l$ agents playing in as $n_{l, i n}$. We consider two cases: (CEL - i) there is at least one agent $k \in N_{h}$ who plays in (CEL ii) there is no agent $k \in N_{h}$ who plays in.

(CEL - i) If there is at least one agent $k \in N_{h}$ who plays in, Corollary 2 , the equilibrium property and our tie-breaking assumption imply that

$$
\begin{aligned}
& V_{i, \text { out }} \leq V_{i, \text { in }}^{e}\left(C E L, n_{h, \text { in }}, n_{l, \text { in }}\right) \text { and } \\
& V_{j, \text { in }}^{e}\left(C E L, n_{h, \text { in }}+1, n_{l, \text { in }}\right)<V_{j, \text { out }} .
\end{aligned}
$$

Now assume that player $j$ switches to $i n$. The number of type $l$ agents playing in is $n_{l}+1$ now. The definition of $C E L$, the equilibrium property, and our tie-breaking assumption imply that

$$
V_{i, \text { in }}^{e}\left(C E L, n_{h, i n}, n_{l, i n}+1\right)=V_{j, i n}^{e}\left(C E L, n_{h, i n}, n_{l, i n}+1\right)<V_{j, \text { out }} \equiv V_{i, \text { out }} .
$$

On the other hand, by Lemma 5,

$$
S_{i}\left(C E L, n_{h, i n}, n_{l, i n}+1\right)>S_{i}\left(C E L, n_{h, i n}, n_{l, i n}\right) .
$$

Hence,

$$
V_{i, i n}^{e}\left(C E L, n_{h, i n}, n_{l, i n}+1\right)>V_{i, i n}^{e}\left(C E L, n_{h, i n}, n_{l, i n}\right) .
$$

Therefore,

$$
\begin{aligned}
V_{i, \text { in }}^{e}\left(C E L, n_{h, \text { in }}, n_{l, i n}\right) & <V_{i, \text { in }}^{e}\left(C E L, n_{h, \text { in }}, n_{l, \text { in }}+1\right) \leq V_{i, \text { out }} \\
\Rightarrow V_{i, \text { in }}^{e}\left(C E L, n_{h, \text { in }}, n_{l, \text { in }}\right) & <V_{i, \text { out }} .
\end{aligned}
$$

However, if the above inequality is satisfied playing in at the beginning cannot be an optimal action for agent $i$; a contradiction. Hence the result follows.

(CEL - ii) If there is no agent $k \in N_{l}$ who plays in under $C E L$ in equilibrium (i.e., $n_{l, i n}=0$ ), this implies

$$
\begin{aligned}
S_{i}\left(C E L, n_{h, i n}, n_{l, i n}\right) & =0 \text { and } \\
V_{i, i n}^{e}\left(C E L, n_{h, i n}, n_{l, i n}\right) & =P_{i}^{e} .
\end{aligned}
$$


However, this implies that $P_{i}^{e} \geq V_{i, o u t}$. Hence, from (P) the result follows.

Now, suppose, by contradiction, that in equilibrium there exists an agent $i \in N_{h}$ who plays in, whereas another agent $j \in N_{h}$ plays out. We consider two cases: (CEL - iii) there is at least one agent $k \in N_{l}$ who plays in (CEL - iv) there is no agent $k \in N_{l}$ who plays $i n$.

(CEL - iii) If there is at least one agent $k \in N_{l}$ who plays $i n$, by Corollary 2,

$$
\begin{aligned}
& \text { if } c_{h}>c_{l}>\lambda_{c e l}, S_{l}(C E L, i n)=\frac{n_{h}}{n_{h}+n_{l}}\left(r-r_{f}\right)\left(w_{l}-w_{h}\right) \text { and } \\
& \text { if } c_{h}>\lambda_{c e l} \geq c_{l}, S_{l}(C E L, i n)=-\left(1+r_{f}\right) w_{l} .
\end{aligned}
$$

Hence, we know that

$$
\begin{aligned}
S_{k}\left(C E L, n_{h, i n}, n_{l, i n}\right) & <0 \text { and } \\
V_{k, \text { in }}^{e}\left(C E L, n_{h, \text { in }}, n_{l, \text { in }}\right) & =P_{k}^{e}+S_{k}\left(C E L, n_{h, \text { in }}, n_{l, \text { in }}\right) \geq V_{k, \text { out }} .
\end{aligned}
$$

Now, assume that agent $j$ switches to playing $i n$. The definition of $C E L$, the equilibrium property and our tie-breaking assumption imply that

$$
V_{j, \text { in }}^{e}\left(C E L, n_{h, \text { in }}+1, n_{l, \text { in }}\right)=V_{i, \text { in }}^{e}\left(C E L, n_{h, \text { in }}+1, n_{l, \text { in }}\right) \leq V_{i, \text { out }} \equiv V_{j, \text { out }}
$$

where,

$$
\begin{aligned}
V_{j, i n}^{e}\left(C E L, n_{h, i n}+1, n_{l, i n}\right) & =P_{j}^{e}+\left(1-\pi_{s}\right) S_{j}\left(C E L, n_{h, i n}+1, n_{l, i n}\right) \text { and } \\
S_{j}\left(C E L, n_{h, i n}+1, n_{l, i n}\right) & >0 .
\end{aligned}
$$

However, if

$$
V_{k, \text { in }}^{e}\left(C E L, n_{h, \text { in }}, n_{l, i n}\right)=P_{k}^{e}+\left(1-\pi_{s}\right) S_{k}\left(C E L, n_{h, \text { in }}, n_{l, \text { in }}\right) \geq V_{k, \text { out }}
$$

then by proportionality,

$$
P_{k}^{e} \geq V_{k, \text { out }} \Leftrightarrow P_{j}^{e} \geq V_{j, \text { out }}
$$

and by Corollary 2 and Lemma 5

$$
S_{k}\left(C E L, n_{h, i n}, n_{l, i n}\right)<0 \text { and } S_{j}\left(C E L, n_{h, i n}, n_{l, i n}+1\right)>0
$$

leads to

$$
V_{j, \text { in }}^{e}\left(C E L, n_{h, \text { in }}, n_{l, \text { in }}+1\right)=V_{i, \text { in }}^{e}\left(C E L, n_{h, \text { in }}, n_{l, \text { in }}+1\right) \leq V_{j, \text { out }},
$$

which is a contradiction. Thus, if it is optimal for a type $l$ agent to play in under $C E L$ in equilibrium, it cannot be optimal for any type $h$ agent to play out in equilibrium, since any type $h$ agent will have non-negative transfers. Hence, the result follows.

(CEL - iv) If there is no agent $k \in N_{l}$ who plays in under $C E L$ in equilibrium (i.e., $n_{l, i n}=0$ ), this implies

$$
\begin{aligned}
S_{i}\left(C E L, n_{h, i n}, n_{l, i n}\right) & =0 \text { and } \\
V_{i, i n}^{e}\left(C E L, n_{h, i n}, n_{l, i n}\right) & =P_{i, i n}^{e} .
\end{aligned}
$$


Then if agent $j$ switches to playing in, by Lemma 5 ,

$$
S_{k}\left(C E L, n_{h, i n}, n_{l, i n}\right)>S_{k}\left(C E L, n_{h, i n}+1, n_{l, i n}\right) .
$$

Then, there will still be no agent $k \in N_{l}$ who plays $i n$.Hence, the expected payoffs of type $h$ agents will not be affected by the existence of other type $h$ agents playing $i n$, which implies

$$
\begin{aligned}
V_{i, \text { in }}^{e}\left(C E L, n_{h, \text { in }}, n_{l, \text { in }}\right) & =V_{j, \text { in }}^{e}\left(C E L, n_{h, \text { in }}+1, n_{l, \text { in }}\right) \\
& =V_{i, \text { in }}^{e}\left(C E L, n_{h, \text { in }}+1, n_{l, \text { in }}\right) \\
& \leq V_{j, \text { out }} \equiv V_{i, \text { out }} .
\end{aligned}
$$

If the inequality above is satisfied strictly, then playing in at the beginning cannot be an optimal action for agent $i$; a contradiction. If it is satisfied, on the other hand, with equality, then it is not optimal for agent $j$ to play out at the beginning (due to the tie-breaking rule) which is again a contradiction. Hence the result follows.

Proof of Lemma 8. (i) By Corollary 2, $S_{l}(C E A, i n)>0$ and $S_{h}(C E A$, in $)<0$. Therefore, if type $l$ agents (who are favored by $C E A$ ) play out, this implies that

$$
V_{l}^{e}(C E A, \text { in })=P_{l}^{e}+\left(1-\pi_{s}\right) S_{l}(C E A, \text { in })<V_{l, \text { out }} .
$$

Since $S_{l}(C E A$, in $)>0$, this implies that $P_{l}^{e}<V_{l, \text { out }}$. Using proportionality, $P_{l}^{e}<V_{l, \text { out }}$ if and only if $P_{h}^{e}<V_{h, \text { out }}$. However, we know that $S_{h}(C E A$, in $)<0$. Therefore,

$$
V_{h}^{e}(C E A, \text { in })=P_{h}^{e}+\left(1-\pi_{s}\right) S_{h}(C E A, \text { in })>V_{h, \text { out }}
$$

cannot hold. Hence, this implies that playing in cannot be optimal for type $h$ agents (who are disfavored by $C E A$ ), if it is not optimal for type $l$ agents.

(ii) By Corollary $2, S_{h}(C E L, i n)>0$ and $S_{l}(C E L, i n)<0$. Therefore, if type $h$ agents (who are favored by $C E L$ ) play out, this implies that

$$
V_{h}^{e}(C E L, \text { in })=P_{h}^{e}+\left(1-\pi_{s}\right) S_{h}(C E L, \text { in })<V_{h, \text { out }} .
$$

Since $S_{h}(C E L$, in $)>0$, this implies that $P_{h}^{e}<V_{h, \text { out }}$. Using proportionality, $P_{h}^{e}<V_{h, \text { out }}$ if and only if $P_{l}^{e}<V_{l, \text { out }}$. However, we know that $S_{l}(C E L$, in $)<0$. Therefore,

$$
V_{l}^{e}(C E L, i n)=P_{l}^{e}+\left(1-\pi_{s}\right) S_{l}(C E L, \text { in })>V_{l, o u t}
$$

cannot hold. Hence, this implies that playing in cannot be optimal for type $l$ agents (who are disfavored by $C E L$ ), if it is not optimal for type $h$ agents.

\section{C - Multiple Companies and Competition}

Characterization of All Nash Equilibria in All Subgames:

In this appendix, we first characterize the Nash equilibria of all subgames. Since there are two companies moving sequentially and there are three possible bankruptcy rules that can be chosen, there are nine subgames for the agents to play. The following results are used in the characterization 
of all subgame perfect Nash equilibria of the whole game. Also note that every equilibria mentioned below are essentially unique.

$\Gamma^{F_{\bar{m}}, F_{\underline{m}}}=\Gamma^{P, P}$

If for all $t \in\{l, h\}, P_{t}^{e} \geq V_{t, o u t}$, then both type $l$ and $h$ agents invest in a company, in equilibrium. It's a dominant strategy to do so since under $P$, what others do does not affect agent's payoff. Therefore, by assumption 2, half of $n_{h}$ and half of $n_{l}$ invest in $\bar{m}$ and the other halves in $\underline{m}$, in equilibrium. Therefore, $V_{\bar{m}}=V_{\underline{m}}=\left(n_{h} w_{h}+n_{l} w_{l}\right) / 2$.

If for all $t \in\{l, h\}, P_{t}^{e}<V_{t, o u t}$, then no agent invests in a company, in equilibrium. Therefore, $V_{\bar{m}}=V_{\underline{m}}=0$.

$\Gamma^{F_{\bar{m}}, F_{\underline{m}}}=\Gamma^{P, C E A}$

If for all $t \in\{l, h\}, P_{t}^{e} \geq V_{t, o u t}$, then both type $l$ and $h$ agents invest in a company, in equilibrium. Note that $C E A$ is always at least weakly better for type $l$ agents and $P$ is always at least weakly better for type $h$ agents. ${ }^{18}$ Hence, by assumption 1, type $l$ agents invest in $\underline{m}$ and type $h$ agents invest in $\bar{m}$, in equilibrium. Therefore, $V_{\bar{m}}=n_{h} w_{h}$ and $V_{\underline{m}}=n_{l} w_{l}$.

If for all $t \in\{l, h\}, P_{t}^{e}<V_{t, o u t}$, then no agent invests in a company, in equilibrium. Therefore, $V_{\bar{m}}=V_{\underline{m}}=0$.

$\Gamma^{F_{\bar{m}}, F_{\underline{m}}}=\Gamma^{P, C E L}$

If for all $t \in\{l, h\}, P_{t}^{e} \geq V_{t, o u t}$, then both type $l$ and $h$ agents invest in a company, in equilibrium. Note that $C E L$ is always at least weakly better for type $h$ agents and $P$ is always at least weakly better for type $l$ agents. ${ }^{19}$ Hence, by assumption 1, type $h$ agents invest in $\underline{m}$ and type $l$ agents invest in $\bar{m}$, in equilibrium. Therefore, $V_{\bar{m}}=n_{l} w_{l}$ and $V_{\underline{m}}=n_{h} w_{h}$.

If for all $t \in\{l, h\}, P_{t}^{e}<V_{t, o u t}$, then no agent invests in a company, in equilibrium. Therefore, $V_{\bar{m}}=V_{\underline{m}}=0$.

$\Gamma^{F_{\bar{m}}, F_{\underline{m}}}=\Gamma^{C E A, P}$

The analysis in this case is same with the one in $\Gamma^{P, C E A}$ and hence omitted. Only difference is that when for all $t \in\{l, h\}, P_{t}^{e} \geq V_{t, \text { out }}$, type $l$ agents invest in $\bar{m}$ and type $h$ agents invest in $\underline{m}$, in equilibrium. Therefore, now $V_{\bar{m}}=n_{l} w_{l}$ and $V_{\underline{m}}=n_{h} w_{h}$.

$\Gamma^{F_{\bar{m}}, F_{\underline{m}}}=\Gamma^{C E A, C E A}$

If for all $t \in\{l, h\}, P_{t}^{e} \geq V_{t, o u t}$ and $P_{h}^{e}+\left(1-\pi_{s}\right) S_{h}(C E A, i n) \geq V_{h, o u t}$, then both type $l$ and $h$ agents invest in a company, in equilibrium. ${ }^{20}$ Since both companies use $C E A$, agents are indifferent between them. Hence, by assumption 2, half of each type invest in $\bar{m}$ and the other halves invest in $\underline{m}$, in equilibrium. Therefore, $V_{\bar{m}}=V_{m}=\left(n_{h} w_{h}+n_{l} w_{l}\right) / 2$.

If for all $t \in\{l, h\}, P_{t}^{e} \geq V_{t, \text { out }}$ and $P_{h}^{e}+\left(1-\pi_{s}\right) S_{h}(C E A$, in $)<V_{h \text {,out }}$, then only type $l$ agents invest in a company, in equilibrium. Playing in is a weakly dominant strategy for type $l$ agents when $C E A$ is chosen by companies and given that, the best response of type $h$ agents is to play out.

\footnotetext{
${ }^{18}$ Note that if $C E L$ was chosen by one of the companies, it would always be at least weakly better for type $h$ agents. But in the presence of $P$ and $C E A, P$ is the weakly better choice for type $h$ agents.

${ }^{19}$ Note that if $C E A$ was chosen by one of the companies, it would always be at least weakly better for type $l$ agents. But in the presence of $P$ and $C E L, P$ is the weakly better choice for type $l$ agents.

${ }^{20}$ Note that $S_{h}(C E A, i n)$ in the inequality refers to the per-capita transfer a type $h$ agent would make if $n_{h} / 2$ type $h$ agents and $n_{l} / 2$ type $l$ agents invested in a company that has chosen $C E A$. But this is equal to the per-capita transfer a type $h$ agent would make if $n_{h}$ type $h$ agents and $n_{l}$ type $l$ agents invested in that company. Hence, we kept the original notation.
} 
Since both companies use $C E A$, type $l$ agents are indifferent between them. Hence, by assumption 2 , half of type $l$ agents invest in $\bar{m}$ and the other half invest in $\underline{m}$, in equilibrium. Therefore, $V_{\bar{m}}=V_{\underline{m}}=n_{l} w_{l} / 2$.

If for all $t \in\{l, h\}, P_{t}^{e}<V_{t, \text { out }}$, then no agent invests in a company, in equilibrium. Therefore, $V_{\bar{m}}=V_{\underline{m}}=0$.

$\Gamma^{F_{\bar{m}}, F_{\underline{m}}}=\Gamma^{C E A, C E L}$

If for all $t \in\{l, h\}, P_{t}^{e} \geq V_{t, o u t}$, then both type $l$ and $h$ agents invest in a company. Note that $C E L$ is always at least weakly better for type $h$ agents and $C E A$ is always at least weakly better for type $l$ agents. Hence, by assumption 1, type $l$ agents invest in $\underline{m}$ and type $h$ agents invest in $\bar{m}$, in equilibrium. Thus, $V_{\bar{m}}=n_{h} w_{h}$ and $V_{m}=n_{l} w_{l}$.

If for all $t \in\{l, h\}, P_{t}^{e}<V_{t, \text { out }}$, then no agent invests in a company, in equilibrium. Therefore, $V_{\bar{m}}=V_{\underline{m}}=0$.

$\Gamma^{F_{\bar{m}}, F_{\underline{m}}}=\Gamma^{C E L, P}$

The analysis in this case is same with the one in $\Gamma^{P, C E L}$ and hence omitted. Only difference is that when for all $t \in\{l, h\}, P_{t}^{e} \geq V_{t, \text { out }}$, type $l$ agents invest in $\underline{m}$ and type $h$ agents invest in $\bar{m}$, in equilibrium. Therefore, $V_{\bar{m}}=n_{h} w_{h}$ and $V_{m}=n_{l} w_{l}$.

$\Gamma^{F_{\bar{m}}, F_{\underline{m}}}=\Gamma^{C E L, C E A}$

The analysis in this case is same with the one in $\Gamma^{C E A, C E L}$ and hence omitted. Only difference is that when for all $t \in\{l, h\}, P_{t}^{e} \geq V_{t, o u t}$, type $l$ agents invest in $\underline{m}$ and type $h$ agents invest in $\bar{m}$, in equilibrium. Therefore, $V_{\bar{m}}=n_{h} w_{h}$ and $V_{\underline{m}}=n_{l} w_{l}$.

$\Gamma^{F_{\bar{m}}, F_{\underline{m}}}=\Gamma^{C E L, C E L}$

If for all $t \in\{l, h\}, P_{t}^{e} \geq V_{t, \text { out }}$ and $P_{l}^{e}+\left(1-\pi_{s}\right) S_{l}(C E L$, in $) \geq V_{l, \text { out }}$, then both type $l$ and $h$ agents invest in a company, in equilbrium. Since both companies use $C E L$, agents are indifferent between them. Hence, by assumption 2, half of each type invest in $\bar{m}$ and other halves invest in $\underline{m}$, in equilibrium. Therefore, $V_{\bar{m}}=V_{m}=\left(n_{h} w_{h}+n_{l} w_{l}\right) / 2$.

If for all $t \in\{l, h\}, P_{t}^{e} \geq V_{t, \text { out }}$ and $P_{l}^{e}+\left(1-\pi_{s}\right) S_{l}(C E L$, in $)<V_{l, \text { out }}$, then only type $h$ agents invest in a company. Playing in is a weakly dominant strategy for type $h$ agents when $C E L$ is chosen by companies and given that, the best response of type $l$ agents is to play out. Since both companies use $C E L$, type $h$ agents are indifferent between them. Hence, by assumption 2, half of type $h$ agents invest in $\bar{m}$ and other halves invest in $\underline{m}$, in equilibrium. Therefore, $V_{\bar{m}}=V_{\underline{m}}=n_{h} w_{h} / 2$.

If for all $t \in\{l, h\}, P_{t}^{e}<V_{t, o u t}$, then no agent invests in a company, in equilibrium. Therefore, $V_{\bar{m}}=V_{\underline{m}}=0$.

\section{Characterization of All Subgame Perfect Nash Equilibria:}

In this part, we characterize all subgame perfect Nash equilibria of the extended model with two companies by using the results in the previous part. Since representation of equilibria with strategies of all players becomes reader unfriendly (due to the fact that there are three subgames for $\underline{m}$ and nine subgames for agents), we mention only the subgame perfect equilibrium actions and resulting outcomes in this part. 
C1. If for all $t \in\{l, h\}$

$$
\begin{aligned}
P_{t}^{e} & \geq V_{t, \text { out }}, \\
P_{h}^{e}+\left(1-\pi_{s}\right) S_{h}(C E A, \text { in }) & <V_{h, \text { out }}, \text { and } \\
P_{l}^{e}+\left(1-\pi_{s}\right) S_{l}(C E L, \text { in }) & <V_{l, \text { out }},
\end{aligned}
$$

C1.1. $n_{h} w_{h}>n_{l} w_{l}$

C1.1.1. $n_{h} w_{h} / 2<n_{l} w_{l}$ : Since type $h$ agents hold, in total, a larger amount of money and $\underline{m}$ finds it better to attract type $l$ agents instead of sharing type $h$ agents (since $n_{h} w_{h} / 2<n_{l} w_{l}$ ), $\bar{m}$ choosing $C E L$ and $\underline{m}$ choosing either $C E A$ or $P$ are best responses to each other. Moreover, we know from our analysis above that both in $\Gamma^{C E L, P}$ and $\Gamma^{C E L, C E A}$, type $h$ agents invest in $\bar{m}$ and type $l$ agents invest in $\underline{m}$ in equilibrium under the conditions given by $C 1$. Thus, in both subgame perfect Nash equilibria, $V_{\bar{m}}=n_{h} w_{h}$ and $V_{\underline{m}}=n_{l} w_{l}$.

C1.1.2. $n_{h} w_{h} / 2 \geq n_{l} w_{l}$ : Since type $h$ agents hold, in total, a larger amount of money and $\underline{m}$ finds it better to share type $h$ agents than attracting type $l$ agents (since $n_{h} w_{h} / 2 \geq n_{l} w_{l}$ ), $\bar{m}$ and $\underline{m}$ both choosing $C E L$ are best responses to each other. Moreover, we know from our analysis above that in $\Gamma^{C E L, C E L}$, a half of type $h$ agents invest in $\bar{m}$ and the other half invest in $\underline{m}$ whereas type $l$ agents do not invest in any company in equilibrium under the conditions given by $C 1$. Thus, in the unique subgame perfect Nash equilibrium, $V_{\bar{m}}=V_{\underline{m}}=n_{h} w_{h} / 2$.

C1.2. $n_{l} w_{l}>n_{h} w_{h}$

C1.2.1. $n_{l} w_{l} / 2<n_{h} w_{h}$ : Since type $l$ agents hold, in total, a larger amount of money and $\underline{m}$ finds it better to attract type $h$ agents instead of sharing type $l$ agents (since $n_{l} w_{l} / 2<n_{h} w_{h}$ ), $\bar{m}$ choosing $C E A$ and $\underline{m}$ choosing either $C E L$ or $P$ are best responses to each other. Moreover, we know from our analysis above that both in $\Gamma^{C E A, P}$ and $\Gamma^{C E A, C E L}$, type $l$ agents invest in $\bar{m}$ and type $h$ agents invest in $\underline{m}$ in equilibrium under the conditions given by $C 1$. Thus, in both subgame perfect Nash equilibria, $V_{\bar{m}}=n_{l} w_{l}$ and $V_{\underline{m}}=n_{h} w_{h}$.

C1.2.2. $n_{l} w_{l} / 2 \geq n_{h} w_{h}$ : Since type $l$ agents hold, in total, a larger amount of money and $\underline{m}$ finds it better to share type $l$ agents than attracting type $h$ agents (since $n_{l} w_{l} / 2 \geq n_{h} w_{h}$ ), $\bar{m}$ and $\underline{m}$ both choosing $C E A$ are best responses to each other. Moreover, we know from our analysis above that in $\Gamma^{C E A, C E A}$, a half of type $l$ agents invest in $\bar{m}$ and the other half invest in $\underline{m}$ whereas type $h$ agents do not invest in any company in equilibrium under the conditions given by $C 1$. Thus, in the unique subgame perfect Nash equilibrium, $V_{\bar{m}}=V_{\underline{m}}=n_{l} w_{l} / 2$.

C1.3. $n_{h} w_{h}=n_{l} w_{l}$ : Given the income equality between two groups, $\bar{m}$ is indifferent between $P, C E A$ and $C E L . \underline{m}$ 's best response to $\bar{m}$ playing $P$ is playing any one of the three rules; its best response to $\bar{m}$ playing $C E A$ is either playing $P$ or $C E L$; and its best response to $\bar{m}$ playing $C E L$ is either playing $P$ or $C E A$. Hence, any combination of rules except $(C E A, C E A)$ and $(C E L, C E L)$ belongs to a subgame perfect Nash equilibrium. Moreover, we know from our analysis above, in $\Gamma^{P, P}$ a half of each type of agent invests in $\bar{m}$ and the other halves invests in $\underline{m}$. In $\Gamma^{C E L, P}$ and $\Gamma^{C E L, C E A}$, type $h$ agents invest in $\bar{m}$ and type $l$ agents invest in $\underline{m}$; in $\Gamma^{C E A, P}$ and $\Gamma^{C E A, C E L}$, type $l$ agents invest in $\bar{m}$ and type $h$ agents invest in $\underline{m}$. In $\Gamma^{P, \overline{C E A}}$, 
type $h$ agents invest in $\bar{m}$ and type $l$ agents invest in $\underline{m}$ and in $\Gamma^{P, C E L}$, type $l$ agents invest in $\bar{m}$ and type $h$ agents invest in $\underline{m}$.

C2. If for all $t \in\{l, h\}$,

$$
\begin{aligned}
P_{t}^{e} & \geq V_{t, \text { out }}, \\
P_{h}^{e}+\left(1-\pi_{s}\right) S_{h}(C E A, \text { in }) & \geq V_{h, \text { out }}, \text { and } \\
P_{l}^{e}+\left(1-\pi_{s}\right) S_{l}(C E L, \text { in }) & <V_{l, \text { out }},
\end{aligned}
$$

C2.1. $n_{h} w_{h}>n_{l} w_{l}$

C2.1.1. $n_{h} w_{h} / 2<n_{l} w_{l}$ : The analysis is similar to the one in $C 1.1 .1$ and hence omitted.

C2.1.2. $n_{h} w_{h} / 2 \geq n_{l} w_{l}$ : The analysis is similar to the one in $C 1.1 .2$ and hence omitted.

C2.2. $n_{l} w_{l} \geq n_{h} w_{h}$ : Since type $l$ agents hold, in total, a larger amount of money and $\underline{m}$ finds it better to share the whole market than attracting only type $h$ agents (since $n_{l} w_{l} \geq n_{h} w_{h} \Longrightarrow$ $\left.\left(n_{h} w_{h}+n_{l} w_{l}\right) / 2 \geq n_{h} w_{h}\right), \bar{m}$ and $\underline{m}$ both choosing $C E A$ are best responses to each other. Moreover, we know from our analysis above that in $\Gamma^{C E A, C E A}$, a half of each type of agents invest in $\bar{m}$ and the other half invest in $\underline{m}$ in equilibrium under the conditions given by $C 2$. Thus, in the unique subgame perfect Nash equilibrium, $V_{\bar{m}}=V_{\underline{m}}=\left(n_{h} w_{h}+n_{l} w_{l}\right) / 2$.

C2.3. $n_{h} w_{h}=n_{l} w_{l}$ : The analysis similar to the one in $C 1.3$ and hence omitted. The only difference is that any combination of rules except $(C E L, C E L)$ belongs to a subgame perfect Nash equilibrium.

C3. If for all $t \in\{l, h\}$,

$$
\begin{aligned}
P_{t}^{e} & \geq V_{t, \text { out }}, \\
P_{h}^{e}+\left(1-\pi_{s}\right) S_{h}(C E A, \text { in }) & <V_{h, \text { out }}, \text { and } \\
P_{l}^{e}+\left(1-\pi_{s}\right) S_{l}(C E L, \text { in }) & \geq V_{l, \text { out }},
\end{aligned}
$$

C3.1. $n_{h} w_{h} \geq n_{l} w_{l}$ : Since type $h$ agents hold, in total, a larger amount of money and $\underline{m}$ finds it better to share the whole market than attracting only type $l$ agents (since $n_{h} w_{h} \geq n_{l} w_{l} \Longrightarrow$ $\left.\left(n_{h} w_{h}+n_{l} w_{l}\right) / 2 \geq n_{l} w_{l}\right), \bar{m}$ and $\underline{m}$ both choosing $C E L$ are best responses to each other. Moreover, we know from our analysis above that in $\Gamma^{C E L, C E L}$, half of each type of agents invest in $\bar{m}$ and the other half invest in $\underline{m}$ in equilibrium under the conditions given by $C 3$. Thus, in the unique subgame perfect Nash equilibrium, $V_{\bar{m}}=V_{\underline{m}}=\left(n_{h} w_{h}+n_{l} w_{l}\right) / 2$.

C3.2. $n_{l} w_{l}>n_{h} w_{h}$

C3.2.1. $n_{l} w_{l} / 2<n_{h} w_{h}$ : The analysis is similar to the one in $C 1.2 .1$ and hence omitted.

C3.2.2. $n_{l} w_{l} / 2 \geq n_{h} w_{h}$ : The analysis is similar to the one in $C 1.2 .2$ and hence omitted.

C3.3. $n_{h} w_{h}=n_{l} w_{l}$ : The analysis is similar to the one in $C 1.3$ and hence omitted. The only difference is that any combination of rules except $(C E A, C E A)$ belongs to a subgame perfect Nash equilibrium. 
C4. If for all $t \in\{l, h\}$,

$$
\begin{aligned}
P_{t}^{e} & \geq V_{t, \text { out }}, \\
P_{h}^{e}+\left(1-\pi_{s}\right) S_{h}(C E A, \text { in }) & \geq V_{h, \text { out }}, \text { and } \\
P_{l}^{e}+\left(1-\pi_{s}\right) S_{l}(C E L, \text { in }) & \geq V_{l, \text { out }},
\end{aligned}
$$

C4.1. $n_{h} w_{h}>n_{l} w_{l}$ : Since type $h$ agents hold, in total, a larger amount of money and $\underline{m}$ finds it better to share the whole market than attracting only type $l$ agents (since $n_{h} w_{h} \geq n_{l} w_{l} \Longrightarrow$ $\left.\left(n_{h} w_{h}+n_{l} w_{l}\right) / 2 \geq n_{l} w_{l}\right), \bar{m}$ and $\underline{m}$ both choosing $C E L$ are best responses to each other. Moreover, we know from our analysis above that in $\Gamma^{C E L, C E L}$, a half of each type of agents invest in $\bar{m}$ and the other half invest in $\underline{m}$ in equilibrium under the conditions given by $C 4$. Thus, in the unique subgame perfect Nash equilibrium, $V_{\bar{m}}=V_{m}=\left(n_{h} w_{h}+n_{l} w_{l}\right) / 2$.

C4.2. $n_{l} w_{l}>n_{h} w_{h}$ : Since type $l$ agents hold, in total, a larger amount of money and $\underline{m}$ finds it better to share the whole market than attracting only type $h$ agents (since $n_{l} w_{l} \geq n_{h} w_{h} \Longrightarrow$ $\left.\left(n_{h} w_{h}+n_{l} w_{l}\right) / 2 \geq n_{h} w_{h}\right), \bar{m}$ and $\underline{m}$ both choosing $C E A$ are best responses to each other. Moreover, we know from our analysis above that in $\Gamma^{C E A, C E A}$, a half of each type of agents invest in $\bar{m}$ and the other half invest in $\underline{m}$ in equilibrium under the conditions given by $C 4$. Thus, in the unique subgame perfect Nash equilibrium, $V_{\bar{m}}=V_{\underline{m}}=\left(n_{h} w_{h}+n_{l} w_{l}\right) / 2$.

C4.3. $n_{h} w_{h}=n_{l} w_{l}$ : Any combination of rules belongs to a subgame perfect Nash equilibrium under the conditions given by $C 4$. In all of these equilibria, both type $l$ and $h$ agents invest in a company.

C5. If for all $t \in\{l, h\}$,

$$
P_{t}^{e}<V_{t, \text { out }},
$$

Any combination of rules belongs to a subgame perfect Nash equilibrium. In none of these equilibria, agents invest in a company.

The comparative statics in the following appendix sections are used in obtaining the results in Section 6.

D - Comparative Static Analyses on $n_{l}, w_{l}, n_{h}$ and $w_{h}$

\begin{tabular}{|c|c|c|c|c|}
\hline & \multicolumn{2}{|c|}{$S_{h}^{\text {cea }}$} & \multicolumn{2}{c|}{$S_{l}^{\text {cea }}$} \\
\hline & $c_{h}>\lambda_{\text {cea }} \geq c_{l}$ & $c_{h}>c_{l} \geq \lambda_{\text {cea }}$ & $c_{h}>\lambda_{\text {cea }} \geq c_{l}$ & $c_{h}>c_{l} \geq \lambda_{\text {cea }}$ \\
\hline$n_{l}$ & - & - & 0 & - \\
\hline$n_{h}$ & + & + & 0 & + \\
\hline$w_{l}$ & - & + & + & - \\
\hline$w_{h}$ & 0 & - & 0 & + \\
\hline
\end{tabular}

Table D1: The Effect of Changes in $n_{l}, n_{h}, w_{l}$ and $w_{h}$ on $S_{t}^{\text {cea }}$

\begin{tabular}{|c|c|c|c|c|}
\hline & \multicolumn{2}{|c|}{$S_{h}^{\text {cel }}$} & \multicolumn{2}{c|}{$S_{l}^{\text {cel }}$} \\
\hline & $c_{h}>c_{l}>\lambda_{\text {cel }}$ & $c_{h}>\lambda_{\text {cel }} \geq c_{l}$ & $c_{h}>c_{l}>\lambda_{\text {cel }}$ & $c_{h}>\lambda_{\text {cel }} \geq c_{l}$ \\
\hline$n_{l}$ & + & + & + & 0 \\
\hline$n_{h}$ & - & - & - & 0 \\
\hline$w_{l}$ & - & + & + & - \\
\hline$w_{h}$ & + & 0 & - & 0 \\
\hline
\end{tabular}


Table D2: The Effect of Changes in $n_{l}, n_{h}, w_{l}$ and $w_{h}$ on $S_{t}^{c e l}$ E - Comparative static analyses on $r$ and $r_{f}$

\begin{tabular}{|c|c|c|c|c|}
\hline & \multicolumn{2}{|c|}{$S_{h}^{\text {cea }}$} & \multicolumn{2}{c|}{$S_{l}^{\text {cea }}$} \\
\hline & $c_{h}>\lambda_{\text {cea }} \geq c_{l}$ & $c_{h}>c_{l} \geq \lambda_{\text {cea }}$ & $c_{h}>\lambda_{\text {cea }} \geq c_{l}$ & $c_{h}>c_{l} \geq \lambda_{c e a}$ \\
\hline$r$ & - & 0 & + & 0 \\
\hline$r_{f}$ & + & - & - & + \\
\hline
\end{tabular}

Table E1: The Effect of Changes in $r$ and $r_{f}$ on $S_{t}^{c e a}$

\begin{tabular}{|c|c|c|c|c|}
\hline & \multicolumn{2}{|c|}{$S_{h}^{\text {cel }}$} & \multicolumn{2}{c|}{$S_{l}^{\text {cel }}$} \\
\hline & $c_{h}>c_{l}>\lambda_{\text {cel }}$ & $c_{h}>\lambda_{\text {cel }} \geq c_{l}$ & $c_{h}>c_{l}>\lambda_{\text {cel }}$ & $c_{h}>\lambda_{\text {cel }} \geq c_{l}$ \\
\hline$r$ & + & 0 & - & 0 \\
\hline$r_{f}$ & - & + & + & - \\
\hline
\end{tabular}

Table E2: The Effect of Changes in $r$ and $r_{f}$ on $S_{t}^{c e l}$ 